EPJ manuscript No.

(will be inserted by the editor)

\title{
Baryon-baryon and baryon-antibaryon interaction amplitudes in the spin-momentum operator expansion method
}

\author{
A.V. Anisovich ${ }^{1,2}$, V.V. Anisovich ${ }^{2}$, E. Klempt ${ }^{1}$, V.A. Nikonov ${ }^{1,2}$, and A.V. Sarantsev ${ }^{1,2}$ \\ 1 Helmholtz-Institut für Strahlen- und Kernphysik, Universität Bonn, Germany \\ 2 Petersburg Nuclear Physics Institute, Gatchina, 188300 Russia
}

Received: August 15, 2018/

\begin{abstract}
Partial wave scattering amplitudes in baryon-baryon and baryon-antibaryon collisions and amplitudes for the production and decay of baryon resonances are constructed in the framework of the spin-momentum operator expansion method. The approach is relativistically invariant and it allows us to perform combined analyses of different reactions imposing analyticity and unitarity directly. The role of final state interactions (triangle and box diagrams) is discussed.
\end{abstract}

PACS. 11.80.Et Partial-wave analysis - 13.30.-a Decays of baryons - 13.60.Le Meson production 14.20.Gk Baryon resonances with $\mathrm{S}=0$

\section{Introduction}

To understand strong interactions at low and intermediate energies is one of the important tasks when quantum chromodynamics is being studied. At large momentum transfer, QCD can be used efficiently due to the smallness of the strong interaction coupling constant [1; the lowenergy domain can be treated using effective field theories 2. The resonance region is much more difficult to access. Lattice gauge calculations are capable to reproduce the masses of ground state hadrons 3 but excited states and their decay properties are difficult to extract from lattice data. For further progress, systematic experimental information seems to be mandatory to identify the leading mechanisms responsible for the mass spectrum and for the decay amplitudes of strongly interacting particles.

Recently, considerable progress has been achieved in meson spectroscopy, even though a commonly agreed picture has not yet emerged. Recent reviews emphasizing different views can be found in $4,5,6,7$. The main sources of recent progress were the study of reactions with multiparticle final states. The analysis of data on proton-antiproton annihilation at rest resulted in the discovery of a number of particles in the region $1300-1800 \mathrm{MeV}$ 8 14; the investigation of the proton-antiproton annihilation in flight led to a large set of new states over the region 1800-2500 MeV [15]-18]. It appeared that the majority of the newly discovered states are lying on linear trajectories against radial excitation number [19]. Such a pattern was not predicted by the classical quark model of Godfrey and Isgur 20] using a linear confinement potential and additional interactions due to effective one-gluon exchange

Correspondence to: nikonov@iskp.uni-bonn.de forces. More recent calculations based on instanton-induced interactions 21,22 can, however, be tuned (by choosing an appropriate Dirac structure of the confinement potential) to reproduce the observed mass pattern very well. The pattern can be understood, too, within a 5dimensional theory holographically dual to QCD (AdS/ QCD) 23 which predicts masses to proportional to $(N+$ $L)$ where $L$ is the intrinsic orbital angular momentum between quark and antiquark and $N$ a radial quantum number. In the light-quark meson spectrum, practically all expected states are observed. However, there are a few additional states which do not belong to these trajectories. These states are candidates to be of exotic nature, e.g., they could be glueballs or hybrids.

The situation in the baryon sector is in some sense reverse: for baryons, the quark model predicts a much larger number of states than that observed experimentally. So far, the pattern seems to suggest that not all degrees of freedom in the three-quark system are realised in the spectrum of excited states. Instead, the pattern of excited states follows the same $(L+N)$ pattern 24] which is observed for mesons. If this is the case, the fact would be an important phenomenon in the physics of highly excited states. Still, a detailed verification of this statement is needed. On the other hand, the main information on baryon resonances has come from the $\pi N$ elastic scattering, and one may hope that many new states will be discovered in (i) reactions involving strangeness in two hadron final states and in (ii) inelastic reactions induced by photons or protons with three or four particles in the final states (for example, two pion photoproduction).

The search for new baryon resonances is of topical interest and several experiments like CB-ELSA, CLAS, 
A.V. Anisovich et al.: Baryon-baryon and baryon-antibaryon interaction ...

GRAAL, SAPHIR, and SPRING-8 pursue active searches using photoproduction as a tool $25,26,27,28,29,30,31,32$, 33, 34, 35, 36, 37, 38, 39, 40, 41, $42,43,44,45,46,47,48,49,50,51$,

52. A few new resonances were suggested $53,54,55$ in fits to these data sets. Proton-proton collision experiments can provide an important source of information on baryon resonances including exotic states (e.g. pentaquarks [56,57, 58, 59,60,61,62,63). COSY at the Research Center Jülich is providing a wealth of data on meson production in proton-proton inelastic scattering $64,65,66,67,68,69,70$,

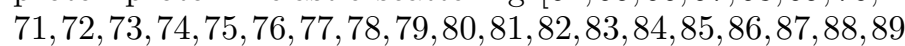
(90, 91, 92, 93, 94, 95, 96 ,97, 98, 99, 100, 101, 102, 103, 104, 105, 106 107,108, 109, 110, 111, 112,113, 114, 115. The experiments Anke and COSY-11 covered mainly the threshold region, while TOF covers the full dynamical range. At present, the upgraded WASA detector is installed at COSY and will provide high-statistics data on the production of neutral mesons in $N N$ interactions $[116$. The data provide stringent information on nucleon-nucleon-meson vertices and on the formation of baryon resonances. Selected papers can be found in [117, 118, 119, 120, 121, 122, 123, 124, 125, 126, 127, 128, 129, 130, 131, 132, 133, 134, 135, 136, 137, 138, 139 140, 141, 142, 143 .

The partial wave analysis of such processes cannot be carried out without taking into account the final state interaction. In many processes, the inclusion of the protonproton interaction dramatically changes the description of the data 144. However, a number of important problems for such analyses has not comprehensively developed yet. Among these problems are a correct treatment of relativistic effects and of the contributions of triangle or box diagrams.

In this paper, we present a relativistically invariant approach for the partial wave analysis of proton-proton interactions. The method is based on the spin-momentum operator expansion suggested in [145, 146, 147, 148]. The contribution of triangle and box diagrams to the meson production processes is discussed and certain examples are considered.

In Section 2, we present the partial wave expansion for baryon-baryon and baryon-antibaryon scattering amplitudes. In Section 3, the unitarity condition for fermionfermion partial wave amplitudes is discussed. The angular momentum and spin operators for nucleon-nucleon scattering are introduced in Section 4, the nucleon-nucleon partial wave amplitude is constructed in Section 5. In this Section, fermion-fermion one-loop diagrams and the cross section for the two-fermion scattering are calculated. The operators for $N \Delta$ production are constructed in the Section 6. Some examples of amplitudes with multi-particle final states are given in Section 7. Properties of the triangle and box diagrams are shortly discussed in Sections 8 and 9 .

\section{Selection rules for baryon-antibaryon and baryon-baryon scattering amplitudes}

\subsection{Baryon pairs with isospin $I=0$}

First, consider the baryon-antibaryon scattering amplitude in a isospin singlet configuration, for example, the $\Lambda \bar{\Lambda}$ scattering amplitude. One can use two alternative representations of the baryon-antibaryon amplitude $\Lambda\left(p_{1}\right) \bar{\Lambda}\left(p_{2}\right)$ $\rightarrow \Lambda\left(p_{1}^{\prime}\right) \bar{\Lambda}\left(p_{2}^{\prime}\right)$.

In the $t$-channel representation the amplitude is the sum of partial waves in the $t$-channel with definite quantum numbers: spin $S$, angular momentum $L$ and total momentum $J$ (we define $\left.t=q^{2}=\left(p_{1}^{\prime}-p_{1}\right)^{2}\right)$ :

$$
\begin{aligned}
& M(s, t, u)=\sum_{\substack{S, L, L^{\prime}, J \\
\mu_{1} \ldots \mu_{J}}}\left(\bar{\psi}\left(p_{1}^{\prime}\right) \tilde{Q}_{\mu_{1} \ldots \mu_{J}}^{S L J}(q) \psi\left(p_{1}\right)\right) \\
& \times\left(\bar{\psi}\left(p_{2}^{\prime}\right) \tilde{Q}_{\mu_{1} \ldots \mu_{J}}^{S L^{\prime} J}(q) \psi\left(p_{2}\right)\right) A_{t}^{\left(S, L^{\prime} L, J\right)}\left(q^{2}\right) .
\end{aligned}
$$

Here, $\tilde{Q}$ is the $t$-channel operator (the four-component spinors $\psi(p)$ are given in Appendix 1) and $\mu_{1}, \mu_{2} \ldots \mu_{J-1}, \mu_{J}$ are the indices of the rank $J$ operator.

Another representation is related to the $s$-channel (we define $\left.s=\left(p_{1}+p_{2}\right)^{2}\right)$ :

$$
\begin{aligned}
& M(s, t, u)=\sum_{\substack{S, L, L^{\prime}, J \\
\mu_{1} \ldots \mu_{J}}}\left(\bar{\psi}\left(p_{1}^{\prime}\right) Q_{\mu_{1} \ldots \mu_{J}}^{S L^{\prime} J}\left(k_{\perp}^{\prime}\right) \psi^{c}\left(-p_{2}^{\prime}\right)\right) \\
& \times\left(\bar{\psi}^{c}\left(-p_{2}\right) Q_{\mu_{1} \ldots \mu_{J}}^{S L J}\left(k_{\perp}\right) \psi\left(p_{1}\right)\right) A_{s}^{\left(S, L^{\prime} L, J\right)}(s) .
\end{aligned}
$$

Here, $\psi^{c}(-p)$ are charge conjugated four-component spinors (see Appendix 1) and $Q_{\mu_{1} \ldots \mu_{J}}^{S L J}$ are the $s$-channel operators, where $S, L, J$ are, correspondingly, spin, angular momentum and total momentum of the partial wave in the $s$-channel. The notations of momenta are as follows:

$$
\begin{aligned}
& P=p_{1}+p_{2}=p_{1}^{\prime}+p_{2}^{\prime}, \quad k=\frac{1}{2}\left(p_{1}-p_{2}\right), \\
& g_{\nu \mu}^{\perp}=g_{\nu \mu}-\frac{P_{\nu} P_{\mu}}{P^{2}} \equiv g_{\mu \nu}^{\perp P}, \quad k_{\perp}=k_{\nu} g_{\nu \mu}^{\perp} .
\end{aligned}
$$

The representation (10) is suitable to consider the $t$ channel meson or Reggeon exchanges, while Eq. (2) is convenient for the $s$-channel partial-wave analysis. The representations (11) and (2) are related to each other by the Fierz transformation [149, with a corresponding reexpansion of the spin-momentum operators.

In terms of the $S L J$ representations, the states are usually described as ${ }^{2 S+1} L_{J}$. The $P$-parity can be calculated as $P=(-1)^{L+1}$ and $C=(-1)^{L+S}$. The states with $S=0$ are unambiguously defined and they form a set of states with $J^{P C}=0^{-+}, 1^{+-}, 2^{-+} \ldots$ The states with $S=1$ and $L=J$ are also uniquely defined and form the set $J^{P C}=1^{++}, 2^{--}, 3^{++} \ldots$ The states with $S=1$ and $L=J-1$ and $L=J+1$ have the same $J^{P C}$ and can mix with each other, that are the states $J^{P C}=0^{++}, 1^{--}, 2^{++}, \ldots$ 


\subsection{Nucleon-antinucleon scattering amplitude}

Let us write the $s$-channel expansion for a pair of nucleons where $N=(p, n)$ forms an isodoublet. The systems $p \bar{n}$ and $n \bar{p}$ have isospin $I=1$, and the $s$-channel expansions of their scattering amplitudes are determined by formulae which are analogous to those for $\Lambda \bar{\Lambda}$, Eq. (2). The systems $p \bar{p}$ and $n \bar{n}$ are a superposition of two states, with $I=0$ and $I=1$. The nucleon-antinucleon amplitudes read:

$$
\begin{aligned}
& \boldsymbol{p}\left(\boldsymbol{p}_{\mathbf{1}}\right) \overline{\boldsymbol{n}}\left(\boldsymbol{p}_{\mathbf{2}}\right) \rightarrow \boldsymbol{p}\left(\boldsymbol{p}_{\mathbf{1}}^{\prime}\right) \overline{\boldsymbol{n}}\left(\boldsymbol{p}_{\mathbf{2}}^{\prime}\right)(\boldsymbol{I}=\mathbf{1}): \\
& \left(C_{1 / 2}^{11} 1 / 2,1 / 21 / 2\right)^{2} M_{1}(s, t, u)=M_{1}(s, t, u) \\
& \boldsymbol{p}\left(\boldsymbol{p}_{\mathbf{1}}\right) \overline{\boldsymbol{p}}\left(\boldsymbol{p}_{\mathbf{2}}\right) \rightarrow \boldsymbol{p}\left(\boldsymbol{p}_{\mathbf{1}}^{\prime}\right) \overline{\boldsymbol{p}}\left(\boldsymbol{p}_{\mathbf{2}}^{\prime}\right)(\boldsymbol{I}=\mathbf{0}, \mathbf{1}): \\
& \quad\left(C_{1 / 2}^{10} 1 / 2,1 / 2-1 / 2\right)^{2} M_{1}(s, t, u) \\
& \quad+\left(C_{1 / 2}^{00} 1 / 2,1 / 2-1 / 2\right)^{2} M_{0}(s, t, u)= \\
& \quad \frac{1}{2} M_{1}(s, t, u)+\frac{1}{2} M_{0}(s, t, u), \\
& \boldsymbol{p}\left(\boldsymbol{p}_{\mathbf{1}}\right) \overline{\boldsymbol{p}}\left(\boldsymbol{p}_{\mathbf{2}}\right) \rightarrow \boldsymbol{n}\left(\boldsymbol{p}_{\mathbf{1}}^{\prime}\right) \overline{\boldsymbol{n}}\left(\boldsymbol{p}_{\mathbf{2}}^{\prime}\right)(\boldsymbol{I}=\mathbf{0 , 1}): \\
& C_{1 / 2}^{10} 1 / 2,1 / 2-1 / 2 \\
& +C_{1 / 2}^{00} C_{1 / 2}^{10}-1 / 2,1 / 21 / 2 M_{1}(s, t, u) \\
& \frac{1}{2} M_{1}(s, t, u)-\frac{1}{2} M_{0}(s, t, u) .
\end{aligned}
$$

Note that, by writing the $N \bar{N}$ (or $N N$ ) scattering amplitudes, one can use alternatively either isotopic Pauli matrices $(I / \sqrt{2}, \tau / \sqrt{2})$ or Clebsch-Gordan coefficients. In (44), we use Clebsch-Gordan coefficients which allows us to consider reactions in which states with $I>1 / 2$ are produced.

The $s$-channel operator expansion for $N \bar{N} \rightarrow N \bar{N}$ can be written as

$$
\begin{aligned}
& M_{I}(s, t, u)=\sum_{\substack{S, L, L^{\prime}, J \\
\mu_{1} \ldots \mu_{J}}}\left(\bar{\psi}\left(p_{1}^{\prime}\right) Q_{\mu_{1} \ldots \mu_{J}}^{S L^{\prime} J}\left(k^{\prime}\right) \psi^{c}\left(-p_{2}^{\prime}\right)\right) \\
& \times\left(\bar{\psi}^{c}\left(-p_{2}\right) Q_{\mu_{1} \ldots \mu_{J}}^{S L J}(k) \psi\left(p_{1}\right)\right) A_{I}^{\left(S, L^{\prime} L, J\right)}(s) .
\end{aligned}
$$

Since the two masses are equal, $k=k_{\perp}$ holds. In Eq. (7), the summation is performed over all states (as well as for the $\Lambda \bar{\Lambda}$ scattering amplitude). The spin-momentum operators $Q_{\mu_{1} \ldots \mu_{J}}^{S L J}(k)$ for the states with $J=0,1,2$ are given in Section 4.

\subsection{Amplitude for $p \Lambda \rightarrow p \Lambda$ scattering}

It is convenient to present the amplitude $p \Lambda \rightarrow p \Lambda$ precisely in the same technique which was used in the consideration of the $s$-channel fermion-antifermion system. To this aim, we declare $p$ being a fermion and $\Lambda$ an antifermion. Then, the $s$-channel expansion for the $p \Lambda \rightarrow p \Lambda$ scattering amplitude reads:

$$
\begin{aligned}
& M_{N \Lambda \rightarrow N \Lambda}(s, t, u)=\sum_{\substack{S, L, L^{\prime}, J \\
\mu_{1} \ldots \mu_{J}}}\left(\bar{\psi}_{N}\left(p_{1}^{\prime}\right) Q_{\mu_{1} \ldots \mu_{J}}^{S L^{\prime} J}\left(k_{\perp}^{\prime}\right) \psi_{\Lambda}^{c}\left(-p_{2}^{\prime}\right)\right) \\
& \times\left(\bar{\psi}_{\Lambda}^{c}\left(-p_{2}\right) Q_{\mu_{1} \ldots \mu_{J}}^{S L J}\left(k_{\perp}\right) \psi_{N}\left(p_{1}\right)\right) A_{N \Lambda \rightarrow N \Lambda}^{\left(S, L^{\prime} L, J\right)}(s) .
\end{aligned}
$$

\subsection{Amplitude for $\Lambda \Lambda \rightarrow \Lambda \Lambda$ scattering}

Let us present the amplitude $\Lambda \Lambda \rightarrow \Lambda \Lambda$ in the technique which was used for reaction $p \Lambda \rightarrow p \Lambda$. So, we declare the 1st $\Lambda$ to be a fermion and the 2 nd one to be an antifermion. One can distinguish between them, for example, in the c.m. system labeling a particle scattered into the backward hemisphere as "antifermion". Then the $s$ channel expansion for the $\Lambda \Lambda \rightarrow \Lambda \Lambda$ scattering amplitude reads:

$$
\begin{aligned}
& M_{\Lambda \Lambda \rightarrow \Lambda \Lambda}(s, t, u)=\sum_{\substack{S, L, L^{\prime}, J \\
\mu_{1} \ldots \mu_{J}}}\left(\bar{\psi}_{\Lambda}\left(p_{1}^{\prime}\right) Q_{\mu_{1} \ldots \mu_{J}}^{S L^{\prime} J}\left(k^{\prime}\right) \psi_{\Lambda}^{c}\left(-p_{2}^{\prime}\right)\right) \\
& \times\left(\bar{\psi}_{\Lambda}^{c}\left(-p_{2}\right) Q_{\mu_{1} \ldots \mu_{J}}^{S L J}(k) \psi_{\Lambda}\left(p_{1}\right)\right) A_{\Lambda \Lambda \rightarrow \Lambda \Lambda}^{\left(S, L^{\prime} L, J\right)}(s) .
\end{aligned}
$$

In this reaction, a selection rule for quantum numbers caused by the Fermi statistics should be taken into account, such as:

$$
(-1)^{S+L+1}=-1 .
$$

Therefore, the following states contribute into (9) only:

$$
\begin{aligned}
& S=1:(L=1 ; J=1),(L=3 ; J=2,3,4), \ldots \\
& S=0:(L=0 ; J=0),(L=2 ; J=2), \ldots
\end{aligned}
$$

\subsection{Nucleon-nucleon scattering amplitude}

Nucleon is an isodoublet with components $p \rightarrow(I=$ $\left.1 / 2, I_{3}=1 / 2\right)$ and $n \rightarrow\left(I=1 / 2, I_{3}=-1 / 2\right)$. The systems $p p$ and $n n$ have total isospin $I=1$, and the $s$-channel expansions of their scattering amplitudes are determined by formulae analogous to those for $\Lambda \Lambda$, Eq. (9). The system $p n$ is a superposition of two states, with total isospins $I=0$ and $I=1$. The amplitudes read:

$$
\begin{aligned}
& \boldsymbol{p}\left(\boldsymbol{p}_{\mathbf{1}}\right) \boldsymbol{p}\left(\boldsymbol{p}_{\mathbf{2}}\right) \rightarrow \boldsymbol{p}\left(\boldsymbol{p}_{\mathbf{1}}^{\prime}\right) \boldsymbol{p}\left(\boldsymbol{p}_{\mathbf{2}}^{\prime}\right)(\boldsymbol{I}=\mathbf{1}): \\
& \quad\left(C_{1 / 2}^{11}{ }_{1 / 2}, 1 / 21 / 2\right)^{2} M_{1}(s, t, u)=M_{1}(s, t, u), \\
& \boldsymbol{p}\left(\boldsymbol{p}_{\mathbf{1}}\right) \boldsymbol{n}\left(\boldsymbol{p}_{\mathbf{2}}\right) \rightarrow \boldsymbol{p}\left(\boldsymbol{p}_{\mathbf{1}}^{\prime}\right) \boldsymbol{n}\left(\boldsymbol{p}_{\mathbf{2}}^{\prime}\right)(\boldsymbol{I}=\mathbf{0}, \mathbf{1}): \\
& \quad\left(C_{1 / 2}^{10} 1 / 2,1 / 2-1 / 2\right)^{2} M_{1}(s, t, u) \\
& \quad+\left(C_{1 / 2}^{00} 1 / 2,1 / 2-1 / 2\right)^{2} M_{0}(s, t, u)= \\
& \quad \frac{1}{2} M_{1}(s, t, u)+\frac{1}{2} M_{0}(s, t, u) \\
& \boldsymbol{n}\left(\boldsymbol{p}_{\mathbf{1}}\right) \boldsymbol{n}\left(\boldsymbol{p}_{\mathbf{2}}\right) \rightarrow \boldsymbol{n}\left(\boldsymbol{p}_{\mathbf{1}}^{\prime}\right) \boldsymbol{n}\left(\boldsymbol{p}_{\mathbf{2}}^{\prime}\right)(\boldsymbol{I}=\mathbf{1}): \\
& \left(C_{1 / 2-1 / 2,1 / 2-1 / 2}^{1-1}\right)^{2} M_{1}(s, t, u)=M_{1}(s, t, u) .
\end{aligned}
$$

The $s$-channel operator expansion gives for $M_{I}(s, t, u)$ in the reaction $p n \rightarrow p n(I=0)$ :

$$
\begin{gathered}
M_{0}(s, t, u)=\sum_{\substack{S, L, L^{\prime}, J \\
\mu_{1} \ldots \mu_{J}}}\left(\bar{\psi}_{p}\left(p_{1}^{\prime}\right) Q_{\mu_{1} \ldots \mu_{J}}^{S L^{\prime} J}\left(k^{\prime}\right) \psi_{n}^{c}\left(-p_{2}^{\prime}\right)\right) \\
\times\left(\bar{\psi}_{n}^{c}\left(-p_{2}\right) Q_{\mu_{1} \ldots \mu_{J}}^{S L J}(k) \psi_{p}\left(p_{1}\right)\right) A_{0}^{\left(S, L^{\prime} L, J\right)}(s), \\
S=1:(L=0 ; J=1),(L=2 ; J=1,2,3), \ldots \\
S=0:(L=1 ; J=1),(L=3 ; J=3), \ldots
\end{gathered}
$$


and for $I=1$ :

$$
\begin{aligned}
M_{1}(s, t, u)=\sum_{\substack{S, L, L^{\prime}, J \\
\mu_{1} \ldots \mu_{J}}}\left(\bar{\psi}_{p}\left(p_{1}^{\prime}\right) Q_{\mu_{1} \ldots \mu_{J}}^{S L^{\prime} J}\left(k^{\prime}\right) \psi_{n}^{c}\left(-p_{2}^{\prime}\right)\right) \\
\quad \times\left(\bar{\psi}_{n}^{c}\left(-p_{2}\right) Q_{\mu_{1} \ldots \mu_{J}}^{S L L}(k) \psi\left(p_{1}\right)\right) A_{1}^{\left(S, L^{\prime} L, J\right)}(s), \\
S=1: \quad(L=1 ; J=0,1,2),(L=3 ; J=2,3,4), \ldots \\
S=0: \quad(L=0 ; J=0),(L=2 ; J=2), \ldots
\end{aligned}
$$

The selection rule for quantum numbers in (15) and (16) is caused by the Fermi statistics.

Analogous partial wave expansions can be written for the reactions $p p \rightarrow p p$ and $n n \rightarrow n n(I=1)$, with an obvious replacing in (16): $n \rightarrow p$ for $p p \rightarrow p p$ and $p \rightarrow n$ for $n n \rightarrow n n$. Here, as for $\Lambda \Lambda \rightarrow \Lambda \Lambda$, declaring one nucleon as a fermion and the second one as antifermion, one distinguishes between them in c.m. system labeling a particle scattered into the backward hemisphere as "antifermion".

\section{Unitarity conditions and $\boldsymbol{K}$-matrix representations of baryon-antibaryon and baryon-baryon scattering amplitudes}

Here, we write down the unitarity conditions and give the $K$-matrix representations of the baryon-antibaryon and baryon-baryon scattering amplitudes suggesting that inelastic processes are switched off (for example, because the energy is not large enough). Generalisation of the $K$ matrix representations in case when inelastic channels are switched on can be performed in a standard way.

\section{$3.1 \Lambda \bar{\Lambda}$ scattering}

In this subsection, we consider the unitarity condition for the amplitude with $J=L$. The generalisation for $J=$ $L \pm 1$ amplitude is considered in the last subsection. For the amplitude $\Lambda \bar{\Lambda} \rightarrow \Lambda \bar{\Lambda}$ of Eq. (2), the $s$-channel unitarity condition reads for $J=L$ (we re-define $A_{s}^{(S, L L, J)}(s) \rightarrow$ $\left.A_{\Lambda \bar{\Lambda} \rightarrow \Lambda \bar{\Lambda}}^{(S, L L, J)}(s)\right)$ as follows:

$$
\begin{aligned}
& \sum_{\mu_{1} \ldots \mu_{J}}\left(\bar{\psi}\left(p_{1}^{\prime}\right) Q_{\mu_{1} \ldots \mu_{J}}^{S L J}\left(k^{\prime}\right) \psi^{c}\left(-p_{2}^{\prime}\right)\right) \\
& \times\left(\bar{\psi}^{c}\left(-p_{2}\right) Q_{\mu_{1} \ldots \mu_{J}}^{S L J}(k) \psi\left(p_{1}\right)\right) \operatorname{Im} A_{\Lambda \bar{\Lambda} \rightarrow \Lambda \bar{\Lambda}}^{(S, L L, J)}(s)= \\
& \int d \Phi_{2}\left(p_{1}^{\prime \prime}, p_{2}^{\prime \prime}\right) \sum_{j, \ell} \sum_{\mu_{1} \ldots \mu_{J}}\left(\bar{\psi}\left(p_{1}^{\prime}\right) Q_{\mu_{1} \ldots \mu_{J}}^{S L J}\left(k^{\prime}\right) \psi^{c}\left(-p_{2}^{\prime}\right)\right) \\
& \times\left(\bar{\psi}_{\ell}^{c}\left(-p_{2}^{\prime \prime}\right) Q_{\mu_{1} \ldots \mu_{J}}^{S L J}\left(k^{\prime \prime}\right) \psi_{j}\left(p_{1}^{\prime \prime}\right)\right) A_{\Lambda \bar{\Lambda} \rightarrow \Lambda \bar{\Lambda}}^{(S, L L, J)}(s) \\
& \times \sum_{\mu_{1}^{\prime \prime} \ldots \mu_{J}^{\prime \prime}}\left[\left(\bar{\psi}\left(p_{1}\right) Q_{\mu_{1}^{\prime \prime} \ldots \mu_{J}^{\prime \prime}}^{S L J}(k) \psi^{c}\left(-p_{2}\right)\right)\right. \\
& \left.\times\left(\bar{\psi}_{\ell}^{c}\left(-p_{2}^{\prime \prime}\right) Q_{\mu_{1}^{\prime \prime} \ldots \mu_{J}^{\prime \prime}}^{S L J}\left(k^{\prime \prime}\right) \psi_{j}\left(p_{1}^{\prime \prime}\right) A_{\Lambda \bar{\Lambda} \rightarrow \Lambda \bar{\Lambda}}^{(S, L L, J)}(s)\right)\right]^{+} \cdot(1
\end{aligned}
$$

Finally, one has:

$$
\operatorname{Im} A_{\Lambda \bar{\Lambda} \rightarrow \Lambda \bar{\Lambda}}^{(S, L L, J)}(s)=\rho_{\Lambda \bar{\Lambda}}^{(S L J)}(s) A_{\Lambda \bar{\Lambda} \rightarrow \Lambda \bar{\Lambda}}^{(S, L L, J)^{*}}(s) A_{\Lambda \bar{\Lambda} \rightarrow \Lambda \bar{\Lambda}}^{(S, L L, J)}(s),(18
$$

where

$$
\begin{aligned}
& O_{\mu_{1}^{\prime \prime} \ldots \mu_{J}^{\prime \prime}}^{\mu_{1} \ldots \mu_{J}} \rho_{\Lambda \bar{\Lambda}}^{(S L J)}(s)=\int d \Phi_{2}\left(p_{1}^{\prime \prime}, p_{2}^{\prime \prime}\right) \\
& \times \operatorname{Sp}\left(Q_{\mu_{1} \ldots \mu_{J}}^{S L J}\left(k^{\prime \prime}\right)\left(-\hat{p}_{2}^{\prime \prime}+m_{\Lambda}\right) Q_{\mu_{1}^{\prime \prime} \ldots \mu_{J}^{\prime \prime}}^{S L J}\left(k^{\prime \prime}\right)\left(\hat{p}_{1}^{\prime \prime}+m_{\Lambda}\right)\right) .
\end{aligned}
$$

The projection operator $O_{\mu_{1}^{\prime \prime} \ldots \mu_{J}^{\prime \prime}}^{\mu_{1} \ldots \mu_{J}}$ is presented in Section 4 . The phase space is determined as

$$
\begin{aligned}
& d \tilde{\Phi}_{2}\left(p_{1}, p_{2}\right)=\frac{2 \pi^{4}}{2} d \Phi_{2}\left(p_{1}, p_{2}\right)= \\
& \quad \frac{1}{2}(2 \pi)^{4} \delta^{(4)}\left(P-p_{1}-p_{2}\right) \frac{d^{3} p_{1}}{(2 \pi)^{3} 2 p_{10}} \frac{d^{3} p_{2}}{(2 \pi)^{3} 2 p_{20}} .
\end{aligned}
$$

The projection operator $O_{\mu_{1}^{\prime \prime} \ldots \mu_{J}^{\prime \prime}}^{\mu_{1} \ldots \mu_{J}}$ obeys the convolution rule, $O_{\mu_{1} \ldots \mu_{J}}^{\mu_{1} \ldots \mu_{J}}=2 J+1$, that gives:

$$
\begin{aligned}
& \rho_{\Lambda \bar{\Lambda}}^{(S L J)}(s)=\frac{1}{2 J+1} \int d \tilde{\Phi}_{2}\left(p_{1}^{\prime \prime}, p_{2}^{\prime \prime}\right) \\
& \times \operatorname{Sp}\left(Q_{\mu_{1} \ldots \mu_{J}}^{S L J}\left(k^{\prime \prime}\right)\left(-\hat{p}_{2}^{\prime \prime}+m_{\Lambda}\right) Q_{\mu_{1} \ldots \mu_{J}}^{S L J}\left(k^{\prime \prime}\right)\left(\hat{p}_{1}^{\prime \prime}+m_{\Lambda}\right)\right) .
\end{aligned}
$$

The unitarity condition (18) results in the following $K$ matrix representation of the amplitude $\Lambda \bar{\Lambda} \rightarrow \Lambda \bar{\Lambda}$ :

$$
A_{\Lambda \bar{\Lambda} \rightarrow \Lambda \bar{\Lambda}}^{(S, L L, J)}(s)=\frac{K_{\Lambda \bar{\Lambda} \rightarrow \Lambda \bar{\Lambda}}^{(S, L L, J)}(s)}{1-i \rho_{\Lambda \bar{\Lambda}}^{(S L J)}(s) K_{\Lambda \bar{\Lambda} \rightarrow \Lambda \bar{\Lambda}}^{(S, L L, J)}(s)} .
$$

\section{$3.2 \Lambda \Lambda$ scattering}

Likewise, we consider the unitarity condition for the $\Lambda \Lambda$ scattering amplitude. The $s$-channel unitarity condition for the amplitude $\Lambda \Lambda \rightarrow \Lambda \Lambda$ with $J=L$ reads

$$
\operatorname{Im} A_{\Lambda \Lambda \rightarrow \Lambda \Lambda}^{(S, L L, J)}(s)=\frac{1}{2} \rho_{\Lambda \Lambda}^{(S L J)}(s) A_{\Lambda \Lambda \rightarrow \Lambda \Lambda}^{(S, L L, J)^{*}}(s) A_{\Lambda \Lambda \rightarrow \Lambda \Lambda}^{(S, L L, J)}(s),
$$

where the identity factor $1 / 2$ is introduced. In this way, we keep the definition (20) for $d \tilde{\Phi}_{2}\left(p_{1}^{\prime \prime}, p_{2}^{\prime \prime}\right)$.

We have

$$
\begin{aligned}
& O_{\mu_{1}^{\prime \prime} \ldots \mu_{J}^{\prime \prime}}^{\mu_{1} \ldots \mu_{J}} \rho_{\Lambda \Lambda}^{(S L J)}(s)=\int d \tilde{\Phi}_{2}\left(p_{1}^{\prime \prime}, p_{2}^{\prime \prime}\right) \\
& \times \operatorname{Sp}\left(Q_{\mu_{1} \ldots \mu_{J}}^{S L J}\left(k^{\prime \prime}\right)\left(-\hat{p}_{2}^{\prime \prime}+m_{\Lambda}\right) Q_{\mu_{1}^{\prime \prime} \ldots \mu_{J}^{\prime \prime}}^{S L J}\left(k^{\prime \prime}\right)\left(\hat{p}_{1}^{\prime \prime}+m_{\Lambda}\right)\right) .
\end{aligned}
$$

The convolution rule $O_{\mu_{1} \ldots \mu_{J}}^{\mu_{1} \ldots \mu_{J}}=2 J+1$, gives us

$$
\begin{aligned}
& \rho_{\Lambda \Lambda}^{(S L J)}(s)=\frac{1}{2 J+1} \int d \tilde{\Phi}_{2}\left(p_{1}^{\prime \prime}, p_{2}^{\prime \prime}\right) \\
& \times \operatorname{Sp}\left(Q_{\mu_{1} \ldots \mu_{J}}^{S L J}\left(k^{\prime \prime}\right)\left(-\hat{p}_{2}^{\prime \prime}+m_{\Lambda}\right) Q_{\mu_{1} \ldots \mu_{J}}^{S L J}\left(k^{\prime \prime}\right)\left(\hat{p}_{1}^{\prime \prime}+m_{\Lambda}\right)\right),
\end{aligned}
$$

thus leading to identical definitions for $\rho_{\Lambda \Lambda}^{(S L J)}(s)$ and $\rho^{(S L J)}(s)$, see (21). The unitarity condition (23) results in the following $K$-matrix representation of the amplitude $\Lambda \Lambda \rightarrow \Lambda \Lambda$ :

$$
A_{\Lambda \Lambda \rightarrow \Lambda \Lambda}^{(S, L L, J)}(s)=\frac{K_{\Lambda \Lambda \rightarrow \Lambda \Lambda}^{(S, L L, J)}(s)}{1-\frac{i}{2} \rho_{\Lambda \Lambda}^{(S L J)}(s) K_{\Lambda \Lambda \rightarrow \Lambda \Lambda}^{(S, L L, J)}(s)} .
$$

Let us emphasize the appearance of the identity factor $1 / 2$ in the denominator of (25). 


\subsection{Nucleon-antinucleon partial wave amplitude}

The $K$-matrix representation for $N \bar{N}$ scattering amplitude is written precisely in the same way as for the $\Lambda \bar{\Lambda}$ case. The only new aspect as compared to $\Lambda \bar{\Lambda}$ is that the $N \bar{N}$ scattering is determined by two isotopic amplitudes, see (5) and (6), with $I=0,1$ :

$$
\begin{aligned}
& \boldsymbol{p} \overline{\boldsymbol{n}} \rightarrow \boldsymbol{p} \overline{\boldsymbol{n}}(\boldsymbol{I}=\mathbf{1}) \quad: \frac{1}{2} M_{1}(s, t, u)+\frac{1}{2} M_{0}(s, t, u), \\
& \boldsymbol{p} \overline{\boldsymbol{p}} \rightarrow \boldsymbol{n} \overline{\boldsymbol{n}}(\boldsymbol{I}=\mathbf{0}, \mathbf{1}): \frac{1}{2} M_{1}(s, t, u)-\frac{1}{2} M_{0}(s, t, u) .
\end{aligned}
$$

Being expanded over the $s$-channel operators $Q_{\mu_{1} \ldots \mu_{J}}^{S L J}(k) \otimes$ $Q_{\mu_{1} \ldots \mu_{J}}^{S L^{\prime} J}\left(k^{\prime}\right)$, these amplitudes are represented through partial wave amplitudes $A_{0}^{\left(S, L^{\prime} L, J\right)}(s)$ and $A_{1}^{\left(S, L^{\prime} L, J\right)}(s)$. The unitarity condition for these amplitudes leads again to a $K$-matrix representation.

As above, we consider here the one-channel amplitude, first for $J=L$. The two-channel amplitudes $(S=1, J=$ $L \pm 1$ ) are presented below in Section 3.4.

The imaginary part of the amplitude $A_{I}^{(S, L L, J)}(s)$ with $I=0,1$ and $J=L$ satisfying the $s$-channel unitarity condition reads

$$
\operatorname{Im} A_{I}^{(S, L L, J)}(s)=\rho_{N \bar{N}}^{(S, L L, J)}(s) A_{I}^{(S, L L, J)^{*}}(s) A_{I}^{(S, L L, J)}(s),
$$

where

$$
\begin{aligned}
& \rho_{N \bar{N}}^{\left(S, L L^{\prime}, J\right)}(s)=\frac{1}{2 J+1} \int d \tilde{\Phi}_{2}\left(p_{1}, p_{2}\right) \\
& \times \operatorname{Sp}\left(Q_{\mu_{1} \ldots \mu_{J}}^{S L J}(k)\left(-\hat{p}_{2}+m_{N}\right) Q_{\mu_{1} \ldots \mu_{J}}^{S L^{\prime} J}(k)\left(\hat{p}_{1}+m_{N}\right)\right) .
\end{aligned}
$$

The unitarity condition (28) gives us the following $K$ matrix representation:

$$
A_{I}^{(S, L L, J)}(s)=\frac{K_{I}^{(S, L L, J)}(s)}{1-i \rho_{N \bar{N}}^{(S, L L, J)}(s) K_{I}^{(S, L L, J)}(s)} .
$$

\subsection{Nucleon-nucleon scattering amplitude}

The $p p$ and $n n$ systems are pure $I=1$ states, while the $p n$ is a superposition of two states with total isospins $I=0$ and $I=1$. The amplitudes read:

$$
\begin{aligned}
p \boldsymbol{p} \rightarrow \boldsymbol{p p}, \boldsymbol{n n} \rightarrow \boldsymbol{n n}(\boldsymbol{I}=\mathbf{1}) & : M_{1}(s, t, u), \\
\boldsymbol{p n} \rightarrow \boldsymbol{p n}(\boldsymbol{I}=\mathbf{0}, \mathbf{1}) & : \frac{1}{2} M_{1}(s, t, u)+\frac{1}{2} M_{0}(s, t, u) .
\end{aligned}
$$

The expansion over $s$-channel operators $Q_{\mu_{1} \ldots \mu_{J}}^{S L J}(k) \otimes$ $Q_{\mu_{1} \ldots \mu_{J}}^{S L^{\prime} J}\left(k^{\prime}\right)$ is a representation of these amplitudes through partial wave amplitudes $A_{0}^{\left(S, L^{\prime} L, J\right)}(s)$ and $A_{1}^{\left(S, L^{\prime} L, J\right)}(s)$. (i) Partial wave amplitudes $N N \rightarrow N N$ for $J=L$. For $J=L$, the amplitude $A_{I}^{(S, L L, J)}(s)$ with $I=0,1$ satisfying the $s$-channel unitarity condition are identical to those for nucleon-antinucleon scattering, eqs. (27) and (28), except for the factor $\frac{1}{2}$ in the amplitude originating from Fermi-Dirac statistics.

$$
A_{I}^{(S, L L, J)}(s)=\frac{K_{I}^{(S, L L, J)}(s)}{1-\frac{i}{2} \rho_{N N}^{(S, L L, J)}(s) K_{I}^{(S, L L, J)}(s)} .
$$

(ii) Partial wave amplitudes for $S=1, J=L \pm 1$. In this case, four partial amplitudes form a $2 \times 2$ matrix given by

$$
\begin{aligned}
& \widehat{A}_{I}^{(S=1, L=J \pm 1, J)}(s)= \\
& \quad\left|\begin{array}{l}
A_{I}^{(S=1, J-1 \rightarrow J-1, J)}(s), A_{I}^{(S=1, J-1 \rightarrow J+1, J)}(s) \\
A_{I}^{(S=1, J+1 \rightarrow J-1, J)}(s), A_{I}^{(S=1, J+1 \rightarrow J+1, J)}(s)
\end{array}\right| .
\end{aligned}
$$

The $K$-matrix representation reads:

$$
\begin{aligned}
& \widehat{A}_{I}^{(S=1, L=J \pm 1, J)}(s)=\widehat{K}_{I}^{(S=1, L=J \pm 1, J)}(s) \\
& \quad \times\left[I-\frac{i}{2} \widehat{\rho}_{N N}^{(S=1, L=J \pm 1, J)}(s) \widehat{K}_{I}^{(S=1, L=J \pm 1, J)}(s)\right]^{-1}
\end{aligned}
$$

with the following definitions:

$$
\begin{aligned}
& \widehat{K}_{I}^{(S=1, L=J \pm 1, J)}(s)= \\
& \left|\begin{array}{l}
K_{I}^{(S=1, J-1 \rightarrow J-1, J)}(s), K_{I}^{(S=1, J-1 \rightarrow J+1, J)}(s) \\
K_{I}^{(S=1, J+1 \rightarrow J-1, J)}(s), K_{I}^{(S=1, J+1 \rightarrow J+1, J)}(s)
\end{array}\right|, \\
& \widehat{\rho}_{N N}^{(S=1, L=J \pm 1, J)}(s)= \\
& \left|\begin{array}{l}
\rho_{N=1, J-1 \rightarrow J-1, J)}^{(S=1)}(s), \rho_{N=1, J-1 \rightarrow J+1, J)}^{(S=N)}(s) \\
\rho_{N N}^{(S=1, J+1 \rightarrow J-1, J)}(s), \rho_{N N}^{(S=1, J+1 \rightarrow J+1, J)}(s)
\end{array}\right| .
\end{aligned}
$$

Note that the matrices $\widehat{\rho}_{I}^{(S=1, L=J \pm 1, J)}(s)$ and $\widehat{K}_{I}^{(S=1, L=J \pm 1, J)}(s)$ are symmetrical:

$$
\begin{aligned}
& \rho_{N N}^{(S=1, J-1 \rightarrow J+1, J)}(s)=\rho_{N N}^{(S=1, J+1 \rightarrow J-1, J)}(s), \\
& K_{I}^{(S=1, J-1 \rightarrow J+1, J)}(s)=K_{I}^{(S=1, J+1 \rightarrow J-1, J)}(s) .
\end{aligned}
$$

Let us emphasize that the definitions of the phase spaces for $N N$ and $N \bar{N}$ systems coincide: $\rho_{N N}^{\left(S, L \rightarrow L^{\prime}, J\right)}(s)=$ $\rho_{N \bar{N}}^{\left(S, L \rightarrow L^{\prime}, J\right)}(s)$. In the equation imposing the unitarity condition (as well as in the $K$-matrix representation), the identity of particles in the $N N$ systems is taken into account directly by the factor $1 / 2$. The unitarity conditions for the $\Lambda \bar{\Lambda}, \Lambda \Lambda$ and $N \bar{N}$ two-channel partial wave amplitudes for $S=1$ and $J=L \pm 1$ are written similarly.

\section{Nucleon-nucleon interaction operators}

In this Section, the proton-proton interaction operators are constructed. These operators are constructed using angular momentum and spin operators, whose properties are discussed below. 


\subsection{Angular momentum operators}

The angular-dependent part of the wave function of the composite state is described by operators constructed using relative momenta of particles and the metric tensor. Such operators (we denote them as $X_{\mu_{1} \ldots \mu_{L}}^{(L)}$, where $L$ is the angular momentum) are called angular momentum operators; they correspond to irreducible representations of the Lorentz group [145,147. They satisfy the following properties 145: (i) Symmetry with respect to permutation of any two indices:

$$
X_{\mu_{1} \ldots \mu_{i} \ldots \mu_{j} \ldots \mu_{L}}^{(L)}=X_{\mu_{1} \ldots \mu_{j} \ldots \mu_{i} \ldots \mu_{L}}^{(L)}
$$

(ii) Orthogonality to the total momentum of the system, $P=k_{1}+k_{2}$ :

$$
P_{\mu_{i}} X_{\mu_{1} \ldots \mu_{i} \ldots \mu_{L}}^{(L)}=0
$$

The traceless property for the summation over two any indices:

$$
g_{\mu_{i} \mu_{j}} X_{\mu_{1} \ldots \mu_{i} \ldots \mu_{j} \ldots \mu_{L}}^{(L)}=0 .
$$

Let us consider a one-loop diagram describing the decay of a composite system into two spinless particles which propagate and then form again a composite system. The decay and formation processes are described by angular momentum operators. Due to the conservation of quantum numbers, this amplitude must vanish for initial and final states with different spin. The S-wave operator is a scalar and can be taken as a unit operator. The $\mathrm{P}$-wave operator is a vector. In the dispersion relation approach, it is sufficient that the imaginary part of the loop diagram, with $\mathrm{S}$ and $\mathrm{P}$-wave operators as vertices, is equal to 0 . In the case of spinless particles this requirement entails

$$
\int \frac{d \Omega}{4 \pi} X_{\mu}^{(1)}=0,
$$

where the integral is taken over the solid angle of the relative momentum. In general, the result of such an integration is proportional to the total momentum of the system $P_{\mu}$ (the only external vector):

$$
\int \frac{d \Omega}{4 \pi} X_{\mu}^{(1)}=\lambda P_{\mu} .
$$

Convolution this expression with $P_{\mu}$ and demanding $\lambda=$ 0 , we obtain the orthogonality condition (37). The orthogonality between the $\mathrm{D}$ - and S-waves is provided by the traceless condition (38); conditions (37), (38) provide the orthogonality for all operators with different angular momenta.

The orthogonality condition (37) is automatically fulfilled if the operators are constructed from the relative momenta $k_{\mu}^{\perp}$ and tensor $g_{\mu \nu}^{\perp}$. Both of them are orthogonal to the total momentum of the system, see eq.(3). In the c.m. system, where $P=\left(P_{0}, \boldsymbol{P}\right)=(\sqrt{s}, 0)$, the vector $k^{\perp}$ is space-like: $k^{\perp}=(0, \boldsymbol{k})$.
The operator for $L=0$ is a scalar (for example a unit operator), and the operator for $L=1$ is a vector, which can be constructed from $k_{\mu}^{\perp}$ only. The orbital angular momentum operators for $L=0$ to 3 are:

$$
\begin{aligned}
X^{(0)} & =1, \quad X_{\mu}^{(1)}=k_{\mu}^{\perp} \\
X_{\mu_{1} \mu_{2}}^{(2)} & =\frac{3}{2}\left(k_{\mu_{1}}^{\perp} k_{\mu_{2}}^{\perp}-\frac{1}{3} k_{\perp}^{2} g_{\mu_{1} \mu_{2}}^{\perp}\right), \\
X_{\mu_{1} \mu_{2} \mu_{3}}^{(3)} & = \\
\frac{5}{2}\left[k_{\mu_{1}}^{\perp} k_{\mu_{2}}^{\perp} k_{\mu_{3}}^{\perp}\right. & \left.-\frac{k_{\perp}^{2}}{5}\left(g_{\mu_{1} \mu_{2}}^{\perp} k_{\mu_{3}}^{\perp}+g_{\mu_{1} \mu_{3}}^{\perp} k_{\mu_{2}}^{\perp}+g_{\mu_{2} \mu_{3}}^{\perp} k_{\mu_{1}}^{\perp}\right)\right] .
\end{aligned}
$$

The operators $X_{\mu_{1} \ldots \mu_{L}}^{(L)}$ for $L \geq 1$ can be written in the form of a recurrent relation:

$$
\begin{aligned}
& X_{\mu_{1} \ldots \mu_{L}}^{(L)}=k_{\alpha}^{\perp} Z_{\mu_{1} \ldots \mu_{L}}^{\alpha}, \\
& Z_{\mu_{1} \ldots \mu_{L}}^{\alpha}=\frac{2 L-1}{L^{2}}\left(\sum_{i=1}^{L} X_{\mu_{1} \ldots \mu_{i-1} \mu_{i+1} \ldots \mu_{L}}^{(L-1)} g_{\mu_{i} \alpha}^{\perp}\right. \\
&\left.-\frac{2}{2 L-1} \sum_{\substack{i, j=1 \\
i<j}}^{L} g_{\mu_{i} \mu_{j}}^{\perp} X_{\mu_{1} \ldots \mu_{i-1} \mu_{i+1} \ldots \mu_{j-1} \mu_{j+1} \ldots \mu_{L} \alpha}^{(L-1)}\right) .
\end{aligned}
$$

The convolution equality reads

$$
X_{\mu_{1} \ldots \mu_{L}}^{(L)} k_{\mu_{L}}^{\perp}=k_{\perp}^{2} X_{\mu_{1} \ldots \mu_{L-1}}^{(L-1)} .
$$

Based on Eq.433) and taking into account the traceless property of $X_{\mu_{1} \ldots \mu_{L}}^{(L)}$, one can write down the orthogonality-normalisation condition for orbital angular operators

$$
\begin{aligned}
& \int \frac{d \Omega}{4 \pi} X_{\mu_{1} \ldots \mu_{L}}^{(L)}\left(k^{\perp}\right) X_{\mu_{1} \ldots \mu_{L^{\prime}}}^{\left(L^{\prime}\right)}\left(k^{\perp}\right)=\delta_{L L^{\prime}} \alpha_{L} k_{\perp}^{2 L}, \\
& \alpha_{L}=\prod_{l=1}^{L} \frac{2 l-1}{l} .
\end{aligned}
$$

Iterating Eq. (42), one obtains the following expression for the operator $X_{\mu_{1} \ldots \mu_{L}}^{(L)}$ :

$$
\begin{aligned}
& X_{\mu_{1} \ldots \mu_{L}}^{(L)}\left(k^{\perp}\right)=\alpha_{L} \quad\left[k_{\mu_{1}}^{\perp} k_{\mu_{2}}^{\perp} k_{\mu_{3}}^{\perp} k_{\mu_{4}}^{\perp} \ldots k_{\mu_{L}}^{\perp}\right. \\
& -\frac{k_{\perp}^{2}}{2 L-1}\left(g_{\mu_{1} \mu_{2}}^{\perp} k_{\mu_{3}}^{\perp} k_{\mu_{4}}^{\perp} \ldots k_{\mu_{L}}^{\perp}\right. \\
& \left.+g_{\mu_{1} \mu_{3}}^{\perp} k_{\mu_{2}}^{\perp} k_{\mu_{4}}^{\perp} \ldots k_{\mu_{L}}^{\perp}+\ldots\right) \\
& +\frac{k_{\perp}^{4}}{(2 L-1)(2 L-3)}\left(g_{\mu_{1} \mu_{2}}^{\perp} g_{\mu_{3} \mu_{4}}^{\perp} k_{\mu_{5}}^{\perp} k_{\mu_{6}}^{\perp} \ldots k_{\mu_{L}}^{\perp}\right. \\
& \left.\left.+g_{\mu_{1} \mu_{2}}^{\perp} g_{\mu_{3} \mu_{5}}^{\perp} k_{\mu_{4}}^{\perp} k_{\mu_{6}}^{\perp} \ldots k_{\mu_{L}}^{\perp}+\ldots\right)+\ldots\right] .
\end{aligned}
$$

\subsection{Projection operators and boson propagator}

The projection operator $O_{\nu_{1} \ldots \nu_{L}}^{\mu_{1} \ldots \mu_{L}}$ is constructed from the metric tensors $g_{\mu \nu}^{\perp}$ and it has the following properties:

$$
\begin{aligned}
& X_{\mu_{1} \ldots \mu_{L}}^{(L)} O_{\nu_{1} \ldots \nu_{L}}^{\mu_{1} \ldots \mu_{L}}=X_{\nu_{1} \ldots \nu_{L}}^{(L)}, \\
& O_{\alpha_{1} \ldots \alpha_{L}}^{\mu_{1} \ldots \mu_{L}} O_{\nu_{1} \ldots \nu_{L}}^{\alpha_{1} \ldots \alpha_{L}}=O_{\nu_{1} \ldots \nu_{L}}^{\mu_{1} \ldots \mu_{L}} .
\end{aligned}
$$


Taking into account the definition of the projection operators (46) and the properties of the $X$-operators (45), we obtain

$$
k_{\mu_{1}} \ldots k_{\mu_{L}} O_{\nu_{1} \ldots \nu_{L}}^{\mu_{1} \ldots \mu_{L}}=\frac{1}{\alpha_{L}} X_{\nu_{1} \ldots \nu_{L}}^{(L)}\left(k^{\perp}\right)
$$

This equation presents the basic property of projection operator: it projects any operator with $L$ indices onto the partial wave operator with angular momentum $L$.

For the lowest states,

$$
\begin{aligned}
& O=1, \quad O_{\nu}^{\mu}=g_{\mu \nu}^{\perp} \\
& O_{\nu_{1} \nu_{2}}^{\mu_{1} \mu_{2}}=\frac{1}{2}\left(g_{\mu_{1} \nu_{1}}^{\perp} g_{\mu_{2} \nu_{2}}^{\perp}+g_{\mu_{1} \nu_{2}}^{\perp} g_{\mu_{2} \nu_{1}}^{\perp}-\frac{2}{3} g_{\mu_{1} \mu_{2}}^{\perp} g_{\nu_{1} \nu_{2}}^{\perp}\right)
\end{aligned}
$$

For higher states, the operator can be calculated using the recurrent expression

$$
\begin{aligned}
& O_{\nu_{1} \ldots \nu_{L}}^{\mu_{1} \ldots \mu_{L}}=\frac{1}{L^{2}}\left(\sum_{i, j=1}^{L} g_{\mu_{i} \nu_{j}}^{\perp} O_{\nu_{1} \ldots \nu_{j}-1 \nu_{j}+1 \ldots \nu_{L}}^{\mu_{1} \ldots \mu_{i-1} \mu_{i+1} \ldots \mu_{L}}\right. \\
& -\frac{4}{(2 L-1)(2 L-3)} \\
& \left.\times \sum_{\substack{i<j \\
k<m}}^{L} g_{\mu_{i} \mu_{j}}^{\perp} g_{\nu_{k} \nu_{m}}^{\perp} O_{\nu_{1} \ldots \nu_{k-1} \nu_{k+1} \ldots \nu_{m-1} \nu_{m+1} \ldots \nu_{L}}^{\mu_{1} \ldots \mu_{i-1} \mu_{i+1} \ldots \mu_{j-1} \mu_{j+1} \ldots \mu_{L}}\right) \text {. }
\end{aligned}
$$

The product of two $X$-operators integrated over solid angle (which is equivalent to an integration over internal momenta) depends on external momenta and the metric tensor only. Therefore, it must be proportional to the projection operator. After straightforward calculations, we obtain

$$
\int \frac{d \Omega}{4 \pi} X_{\mu_{1} \ldots \mu_{L}}^{(L)}\left(k^{\perp}\right) X_{\nu_{1} \ldots \nu_{L}}^{(L)}\left(k^{\perp}\right)=\frac{\alpha_{L} k_{\perp}^{2 L}}{2 L+1} O_{\nu_{1} \ldots \nu_{L}}^{\mu_{1} \ldots \mu_{L}} .
$$

Let us introduce the positive value $|\boldsymbol{k}|^{2}$ :

$$
|\boldsymbol{k}|^{2}=-k_{\perp}^{2}=\frac{\left[s-\left(m_{1}+m_{2}\right)^{2}\right]\left[s-\left(m_{1}-m_{2}\right)^{2}\right]}{4 s} .
$$

In the c.m.s. of the reaction, $\boldsymbol{k}$ is the momentum of a particle. In other systems, we use this definition only in the sense of $|\boldsymbol{k}| \equiv \sqrt{-k_{\perp}^{2}}$; clearly, $|\boldsymbol{k}|^{2}$ is a relativistically invariant positive value. Then, Eq. (50) can be written as

$$
\int \frac{d \Omega}{4 \pi} X_{\mu_{1} \ldots \mu_{L}}^{(L)}\left(k^{\perp}\right) X_{\nu_{1} \ldots \nu_{L}}^{(L)}\left(k^{\perp}\right)=\frac{\alpha_{L}|\boldsymbol{k}|^{2 L}}{2 L+1}(-1)^{L} O_{\nu_{1} \ldots \nu_{L}}^{\mu_{1} \ldots \mu_{L}}
$$

The tensor part of numerator of the boson propagator is defined by the projection operator. Let us write it as

$$
F_{\nu_{1} \ldots \nu_{L}}^{\mu_{1} \ldots \mu_{L}}=(-1)^{L} O_{\nu_{1} \ldots \nu_{L}}^{\mu_{1} \ldots \mu_{L}}
$$

This definition guarantees that the width of a resonance (calculated using the decay vertices) has a positive value.

\subsection{Spin operators of two-fermion systems}

The wave function for fermion particles with the momentum $p$ is described as Dirac bispinor:

$$
\begin{aligned}
& u(p)=\frac{1}{\sqrt{2 m} \sqrt{p_{0}+m}}\left(\begin{array}{c}
\left(p_{0}+m\right) \omega \\
(\boldsymbol{p} \boldsymbol{\sigma}) \omega
\end{array}\right), \\
& \bar{u}(p)=\frac{\left(\omega^{*}\left(p_{0}+m\right),-\omega^{*}(\boldsymbol{p} \boldsymbol{\sigma})\right)}{\sqrt{2 m} \sqrt{p_{0}+m}} .
\end{aligned}
$$

To construct the operators for the two-fermion system, one should also introduce the charge-conjugated bispinors:

$$
\begin{aligned}
& u(-p)=\frac{i}{\sqrt{2 m} \sqrt{p_{0}+m}}\left(\begin{array}{c}
(\boldsymbol{p} \boldsymbol{\sigma}) \omega^{\prime} \\
\left(p_{0}+m\right) \omega^{\prime}
\end{array}\right), \\
& \bar{u}(-p)=-i \frac{\left(\omega^{\prime *}(\boldsymbol{p} \boldsymbol{\sigma}),-\omega^{\prime *}\left(p_{0}+m\right),\right)}{\sqrt{2 m} \sqrt{p_{0}+m}} .
\end{aligned}
$$

Here, the $\omega$ and $\omega^{\prime}$ represent 2-dimensional spinors, $\omega^{*}$ and $\omega^{\prime *}$ are the conjugated and transposed spinors. The normalisation condition can be written as

$$
\begin{aligned}
\bar{u}(p) u(p) & =-\bar{u}(-p) u(-p)=1, \\
\sum_{\text {polarisations }} u(p) \bar{u}(p) & =\frac{m+\hat{p}}{2 m}, \\
\sum_{\text {polarisations }} u(-p) \bar{u}(-p) & =\frac{-m+\hat{p}}{2 m},
\end{aligned}
$$

where $\hat{p}=p^{\mu} \gamma_{\mu}$.

Let us consider a two-fermion system with the total momentum $P=k_{1}+k_{2}$ and relative momentum $k=$ $\left(k_{1}-k_{2}\right) / 2$, where $k_{1}$ and $k_{2}$ are their individual momenta, $P^{2}=s$. For the sake of generality, let the fermions have different masses, $m_{1}$ and $m_{2}$. The two-fermion system can form two possible spin state, $S=0$ (singlet state) and $S=1$ (triplet state). The spin operators for these states act between bispinor and charge-conjugated bispinor, $\bar{u}\left(-k_{1}\right) S^{(i)} u\left(k_{2}\right)$ and have the following form:

$$
S^{(0)}=i \gamma_{5}, \quad S^{(1)}=\gamma_{\mu}^{\perp},
$$

where

$$
\gamma_{\mu}^{\perp}=\gamma_{\nu} g_{\mu \nu}^{\perp}
$$

It should be noted that $u\left(-k_{1}\right)$ and $u\left(k_{2}\right)$ have opposite parities, so $\bar{u}\left(-k_{1}\right) \gamma_{5} u\left(k_{2}\right)$ is a scalar and $\bar{u}\left(-k_{1}\right) u\left(k_{2}\right)$ is a pseudoscalar.

As is shown below, that the $\gamma_{\mu}$ operator leads to the mixture of states with total momentum $L+1$ and $L-1$. So, let us introduce the operator for the pure $S=1$ state:

$$
S_{\text {pure }}^{(1)}=\Gamma_{\alpha}^{\perp}=\gamma_{\beta}\left(g_{\alpha \beta}^{\perp}-\frac{4 s k_{\alpha}^{\perp} k_{\beta}^{\perp}}{M(\sqrt{s}+M)\left(s-\delta^{2}\right)}\right),
$$

where $M=m_{1}+m_{2}$ and $\delta=m_{1}-m_{2}$. In the nonrelativistic limit, this operator is equal to the spin-1 operator $\boldsymbol{\sigma}$ and satisfies the orthogonality of the triplet states with the same parity. 


\subsection{Operators for ${ }^{1} L_{J}$ states}

In case of a singlet spin state, the total angular momentum $J$ is equal to the orbital angular momentum $L$ between the two particles. The ground state of such a system is ${ }^{1} S_{0}$ $\left({ }^{2 S+1} L_{J}\right)$ and corresponding operator is just equal to the spin-0 operator $S^{(0)}$ of Eq.(57). For states with orbital momentum $L$, the operator is constructed as a product of the spin-0 operator $S^{(0)}$ and the angular momentum operator $X_{\mu_{1} \ldots \mu_{J}}$ :

$$
V_{\mu_{1} \ldots \mu_{J}}=\sqrt{\frac{2 J+1}{\alpha_{J}}} i \gamma_{5} X_{\mu_{1} \ldots \mu_{J}}^{(J)}\left(k^{\perp}\right) .
$$

The normalisation factor which is introduced here simplifies the expression for the loop diagram (see below).

\subsection{Operators for ${ }^{3} L_{J}$ states with $J=L$}

The ground state in this series is ${ }^{3} P_{1}$, so one should make a convolution of two vectors, $S_{\mu}^{(1)}$ and $X_{\mu}^{(1)}$ that creates a $J=1$ state (vector state). In this case the vertex operator is equal to $\varepsilon_{\nu_{1} \eta \xi \gamma} \gamma_{\eta} k_{\xi}^{\perp} P_{\gamma}$. For states with higher orbital momenta, one needs to replace $k_{\xi}^{\perp}$ by $X_{\xi \nu_{2} \ldots \nu_{J}}^{(J)}$ and perform a full symmetrisation over $\nu_{1}, \nu_{2}, \ldots, \nu_{J}$ indices, which can be done by a convolution with projection operator $O_{\nu_{1} \ldots \nu_{L}}^{\mu_{1} \ldots \mu_{L}}$. The general form of such a vertex is hence given by

$$
V_{\mu_{1} \ldots \mu_{J}}^{L=J} \sim \varepsilon_{\nu_{1} \eta \xi \gamma} \gamma_{\eta} X_{\xi \nu_{2} \ldots \nu_{J}}^{(J)} P_{\gamma} O_{\nu_{1} \ldots \nu_{J}}^{\mu_{1} \ldots \mu_{J}}
$$

Using eqs. (45) and (47), one has

$$
\begin{aligned}
\varepsilon_{\nu_{1} \eta \xi \gamma} X_{\xi \nu_{2} \ldots \nu_{J}}^{(J)} O_{\nu_{1} \ldots \nu_{J}}^{\mu_{1} \ldots \mu_{J}} & =\varepsilon_{\nu_{1} \eta \xi \gamma} k_{\xi}^{\perp} X_{\nu_{2} \ldots \nu_{J}}^{(J-1)} O_{\nu_{1} \ldots \nu_{J}}^{\mu_{1} \ldots \mu_{J}} \\
& \times \frac{2 J-1}{J}
\end{aligned}
$$

Finally, using Eq. (167) the vertex operator can be written as:

$$
V_{\mu_{1} \ldots \mu_{J}}^{L=J}=\sqrt{\frac{(2 J+1) J}{(J+1) \alpha_{J}}} \frac{i \varepsilon_{\alpha \eta \xi \gamma} \gamma_{\eta} k_{\xi}^{\perp} P_{\gamma} Z_{\mu_{1} \ldots \mu_{J}}^{\alpha}}{\sqrt{s}}
$$

where normalisation parameters are again introduced. Note that due to the property of the antisymmetric tensor $\varepsilon_{\alpha \eta \xi \gamma}$ the vertex given by Eq. (63) does not change, if one replaces $\gamma_{\eta}$ by the pure spin operator $\Gamma_{\eta}$.

\subsection{Operators for ${ }^{3} L_{J}$ states with $L<J$ and $L>J$}

To construct operators for ${ }^{3} L_{J}$ states, one should multiply the spin operator $\gamma_{\alpha}$ by the orbital momentum operator for $L=J+1$. So one has:

$$
V_{\mu_{1} \ldots \mu_{J}}^{L<J} \sim \gamma_{\nu_{1}} X_{\nu_{2} \ldots \nu_{J}}^{(J-1)} O_{\nu_{1} \ldots \nu_{J}}^{\mu_{1} \ldots \mu_{J}}
$$

Using Eq.(167) from Appendix 3, we write the vertex operator in the form

$$
V_{\mu_{1} \ldots \mu_{J}}^{L<J}=\gamma_{\alpha} Z_{\mu_{1} \ldots \mu_{J}}^{\alpha} \sqrt{\frac{J}{\alpha_{J}}},
$$

and for the pure spin operator as

$$
\tilde{V}_{\mu_{1} \ldots \mu_{J}}^{L<J}=\Gamma_{\alpha} Z_{\mu_{1} \ldots \mu_{J}}^{\alpha} \sqrt{\frac{J}{\alpha_{J}}} .
$$

The normalisation constant is chosen to facilitate the calculation of loop diagrams containing such a vertex.

To construct such an operator for $L>J$ one should reduce the number of indices in the orbital operator by a convolution with the spin operator:

$$
V_{\mu_{1} \ldots \mu_{J}}^{L>J}=\gamma_{\alpha} X_{\alpha \mu_{1} \ldots \mu_{J}} \sqrt{\frac{J+1}{\alpha_{J}}}
$$

and for pure spin state:

$$
\tilde{V}_{\mu_{1} \ldots \mu_{J}}^{L>J}=\Gamma_{\alpha} X_{\alpha \mu_{1} \ldots \mu_{J}} \sqrt{\frac{J+1}{\alpha_{J}}} .
$$

\section{Calculation of the $N N \rightarrow N N$ amplitude}

\subsection{Structure of the amplitude}

Let us consider $N\left(q_{1}\right) N\left(q_{2}\right) \rightarrow N\left(k_{1}\right) N\left(k_{2}\right)$ transition amplitude with $q_{1}, q_{2}, k_{1}, k_{2}$ being the nucleon momenta, and $k=\left(k_{1}-k_{2}\right) / 2, q=\left(q_{1}-q_{2}\right) / 2$. In this section the structure of such amplitude for different initial and final states is derived.

We start by considering $N N \rightarrow N N$ amplitudes for a singlet state. For the ${ }^{1} L_{J}$ state with $L=J$, the amplitude is given by

$$
\begin{aligned}
A_{s} & =\bar{u}\left(-q_{2}\right) V_{\mu_{1} \ldots \mu_{J}}(q) u\left(q_{1}\right) \\
& \times F_{\nu_{1} \ldots \nu_{J}}^{\mu_{1} \ldots \mu_{J}} \bar{u}\left(k_{1}\right) V_{\nu_{1} \ldots \nu_{J}}(k) u\left(-k_{2}\right) .
\end{aligned}
$$

For sake of simplicity, we omit here and below the invariant part of the amplitude, which will be considered later on. Using Eq. (60), the amplitude reads

$$
\begin{aligned}
A_{s} & =-\bar{u}\left(-q_{2}\right) \gamma_{5} u\left(q_{1}\right) \bar{u}\left(k_{1}\right) \gamma_{5} u\left(-k_{2}\right) \\
& \times(|\boldsymbol{k}||\boldsymbol{q}|)^{n}(2 J+1) P_{J}(z),
\end{aligned}
$$

where $z=(\boldsymbol{k q}) /(|\boldsymbol{k} \| \boldsymbol{q}|)$ is the cosine of scattering angle in c.m. system.

The transition amplitude for the triplet state ${ }^{3} L_{J}$ with $L<J$ is the following:

$$
\begin{aligned}
A_{t}^{L<J} & =\bar{u}\left(-q_{2}\right) V_{\mu_{1} \ldots \mu_{J}}^{L<J}(q) u\left(q_{1}\right) \\
& \times F_{\nu_{1} \ldots \nu_{J}}^{\mu_{1} \ldots \mu_{J}} \bar{u}\left(k_{1}\right) V_{\nu_{1} \ldots \nu_{J}}^{L<J}(k) u\left(-k_{2}\right) .
\end{aligned}
$$

Using Eq. (167), this amplitude can be written in the form

$$
A_{t}^{L<J}=\sum_{i=1}^{5} f_{i} a_{i}(|\boldsymbol{k} \| \boldsymbol{q}|)^{J-1}
$$


A.V. Anisovich et al.: Baryon-baryon and baryon-antibaryon interaction ...

where

$$
\begin{aligned}
& f_{1}=\bar{u}\left(-q_{2}\right) \gamma_{\mu} u\left(q_{1}\right) \bar{u}\left(k_{1}\right) \gamma_{\mu} u\left(-k_{2}\right), \\
& f_{2}=\bar{u}\left(-q_{2}\right) \hat{q} u\left(q_{1}\right) \bar{u}\left(k_{1}\right) \hat{q} u\left(-k_{2}\right), \\
& f_{3}=\bar{u}\left(-q_{2}\right) \hat{k} u\left(q_{1}\right) \bar{u}\left(k_{1}\right) \hat{k} u\left(-k_{2}\right) \\
& f_{4}=\bar{u}\left(-q_{2}\right) \hat{q} u\left(q_{1}\right) \bar{u}\left(k_{1}\right) \hat{k} u\left(-k_{2}\right), \\
& f_{5}=\bar{u}\left(-q_{2}\right) \hat{k} u\left(q_{1}\right) \bar{u}\left(k_{1}\right) \hat{q} u\left(-k_{2}\right) .
\end{aligned}
$$

Here,

$$
\begin{aligned}
& a_{1}=-\frac{P_{J}^{\prime}(z)}{J}, \quad a_{2}=-\frac{P_{J-1}^{\prime \prime}(z)}{J|\boldsymbol{q}|^{2}}, \quad a_{3}=-\frac{P_{J-1}^{\prime \prime}(z)}{J|\boldsymbol{k}|^{2}}, \\
& a_{4}=\frac{1}{J|\boldsymbol{k}||\boldsymbol{q}|}\left(P_{J-2}^{\prime \prime}(z)-2 P_{J-1}^{\prime}(z)\right), a_{5}=\frac{P_{J}^{\prime \prime}(z)}{J|\boldsymbol{k}||\boldsymbol{q}|} \cdot(74)
\end{aligned}
$$

Likewise, the transition amplitude for triplet state ${ }^{3} L_{J}$ with $L>J$ is as follows:

$$
A_{t}^{L>J}=\sum_{i=1}^{5} f_{i} a_{i}(|\boldsymbol{k} \| \boldsymbol{q}|)^{J+1},
$$

where

$$
\begin{aligned}
& a_{1}=-\frac{P_{J+1}^{\prime}(z)}{J+1}, \quad a_{2}=a_{3}=\frac{P_{J+1}^{\prime \prime}(z)}{J+1}, \\
& a_{4}=-\frac{1}{J+1}\left(P_{J}^{\prime \prime}(z)+(2 J+1) P_{J+1}^{\prime}(z)\right), a_{5}=-\frac{P_{J}^{\prime \prime}(z)}{J+1} .
\end{aligned}
$$

If the spin- 1 operator is defined as $\gamma_{\nu}$, there is a mixture between two triplet amplitudes with $L>J$ and $L<J$. The corresponding transition amplitudes are given by

$$
\begin{aligned}
A_{t}^{m i x}= & V_{\mu_{1} \ldots \mu_{J}}^{L<J}(q)(-1)^{J} O_{\nu_{1} \ldots \nu_{J}}^{\mu_{1} \ldots \mu_{J}} V_{\nu_{1} \ldots \nu_{J}}^{L>J}(k)= \\
& \sum_{i=1}^{5} f_{i} a_{i}|\boldsymbol{k}|^{J+1}|\boldsymbol{q}|^{J-1}, \\
A_{t}^{m i x}= & V_{\mu_{1} \ldots \mu_{J}}^{L>J}(q)(-1)^{J} O_{\nu_{1} \ldots \nu_{J}}^{\mu_{1} \ldots \mu_{J}} \quad V_{\nu_{1} \ldots \nu_{J}}^{L<J}(k)= \\
& \sum_{i=1}^{5} f_{i} a_{i}|\boldsymbol{k}|^{J-1}|\boldsymbol{q}|^{J+1},
\end{aligned}
$$

where

$$
\begin{aligned}
& a_{1}=-\sqrt{\frac{J}{J+1}} \frac{P_{J}^{\prime}(z)}{2 J-1}, \quad a_{2}=\sqrt{\frac{J}{J+1}} \frac{P_{J-1}^{\prime \prime}(z)}{2 J-1}, \quad(78) \\
& a_{3}=\sqrt{\frac{J}{J+1}} \frac{P_{J+1}^{\prime \prime}(z)}{2 J-1}, \quad a_{4}=a_{5}=-\sqrt{\frac{J}{J+1}} \frac{P_{J}^{\prime \prime}(z)}{2 J-1} .
\end{aligned}
$$

If one uses the operators based on the pure spin- 1 operator given by eqs. (66) and (68), the functions $f_{1}, f_{2}, \ldots f_{5}$ are substituted by the new functions $\tilde{f}_{1}, \tilde{f}_{2}, \ldots \tilde{f}_{5}$ as follows:

$$
\tilde{f}_{i}=f_{j} M_{j i}
$$

where the transition matrix $M_{j i}$ is equal to

$$
\left(\begin{array}{ccccc}
1 & 0 & 0 & 0 & 0 \\
-\kappa & \frac{\sqrt{s}}{M} & 0 & 0 & -\kappa\left(k^{\perp} q^{\perp}\right) \\
-\kappa & 0 & \frac{\sqrt{s}}{M} & 0 & -\kappa\left(k^{\perp} q^{\perp}\right) \\
\kappa^{2}\left(k^{\perp} q^{\perp}\right) & -\frac{\sqrt{s} \kappa\left(k^{\perp} q^{\perp}\right)}{M} & -\frac{\sqrt{s} \kappa\left(k^{\perp} q^{\perp}\right)}{M} & \frac{s}{M^{2}} & \kappa^{2}\left(k^{\perp} q^{\perp}\right)^{2} \\
0 & 0 & 0 & 0 & 1
\end{array}\right) .
$$

Then, the transition amplitudes for the ${ }^{3} L_{J}$ triplet state with $L<J$ and $L<J$ are

$$
\begin{aligned}
& A_{t}^{L<J}=\sum_{i, j=1}^{5} f_{j} M_{j i} a_{i}(|\boldsymbol{k} \| \boldsymbol{q}|)^{J-1} \\
& A_{t}^{L>J}=\sum_{i, j=1}^{5} f_{j} M_{j i} a_{i}(|\boldsymbol{k} \| \boldsymbol{q}|)^{J+1} .
\end{aligned}
$$

The transition amplitude for the ${ }^{3} L_{J}$ triplet state with $L=J$ is given by

$$
\begin{aligned}
A_{t}^{L=J} & =\bar{u}\left(-q_{2}\right) V_{\mu_{1} \ldots \mu_{J}}^{L=J}(q) u\left(q_{1}\right) \\
& \times F_{\nu_{1} \ldots \nu_{J}}^{\mu_{1} \ldots \mu_{J}} \bar{u}\left(k_{1}\right) V_{\nu_{1} \ldots \nu_{J}}^{L=J}(k) u\left(-k_{2}\right) .
\end{aligned}
$$

Using expressions given in Appendix 3, this amplitude can be written in the form

$$
A_{t}^{L=J}=\left(f_{1} a_{1}+f_{5} a_{5}+f_{6} a_{6}\right)(|\boldsymbol{k} \| \boldsymbol{q}|)^{J}
$$

where

$$
\begin{aligned}
f_{3} & =\bar{u}\left(-q_{2}\right) \gamma_{\mu} u\left(q_{1}\right) \bar{u}\left(k_{1}\right) \gamma_{\nu} u\left(-k_{2}\right) n_{\mu} n_{\nu}, \\
n_{\mu} & =\frac{\varepsilon_{\mu \alpha \beta \gamma} k_{\alpha} q_{\beta} P_{\gamma}}{\sqrt{s}|\boldsymbol{k}||\boldsymbol{q}|}, \quad a_{1}=-\frac{2 J+1}{(J+1) J} z P_{J}^{\prime}(z), \\
a_{5} & =-\frac{2 J+1}{(J+1) J|\boldsymbol{k}||\boldsymbol{q}|} P_{J}^{\prime}(z), \quad a_{6}=\frac{2 J+1}{(J+1) J} P_{J}^{\prime \prime}(z) .
\end{aligned}
$$

\subsection{One-loop diagrams}

The calculation of one-loop diagrams for different vertex operators is an important step in the construction of a unitary $N N$ amplitude. Let us start from the loop diagram for the singlet state and derive all expressions for the case of different particle masses, $m_{1}$ and $m_{2}$. Taking into account that

$$
S p\left[\gamma_{5}\left(m_{1}+\hat{k}_{1}\right) \gamma_{5}\left(m_{2}-\hat{k}_{2}\right)\right]=2\left(s-\delta^{2}\right),
$$

where $\delta=m_{1}-m_{2}$, the one-loop diagram for the singlet state is equal to

$$
\begin{aligned}
& -\int \frac{d \Omega}{4 \pi} S p\left[V_{\mu_{1} \ldots \mu_{J}}\left(k^{\perp}\right)\left(m_{1}+\hat{k}_{1}\right) V_{\nu_{1} \ldots \nu_{J}}\left(k^{\perp}\right)\left(m_{2}-\hat{k}_{2}\right)\right] \\
& =2\left(s-\delta^{2}\right)|\boldsymbol{k}|^{2 J} O_{\nu_{1} \ldots \nu_{J}}^{\mu_{1} \ldots \mu_{J}}(-1)^{J} .
\end{aligned}
$$

The factor $(-1)$ is related to the fermionic nature of the baryon in the loop.

To calculate one-loop diagrams for different triplet states, the following relations are helpful:

$$
\begin{aligned}
S p\left[\gamma_{\mu}^{\perp}\left(m_{1}+\hat{k}_{1}\right) \gamma_{\nu}^{\perp}\left(m_{2}-\hat{k}_{2}\right)\right] & =2\left(s-\delta^{2}\right) g_{\mu \nu}^{\perp}+8 k_{\mu}^{\perp} k_{\nu}^{\perp}, \\
S p\left[\Gamma_{\mu}\left(m_{1}+\hat{k}_{1}\right) \Gamma_{\nu}\left(m_{2}-\hat{k}_{2}\right)\right] & =2\left(s-\delta^{2}\right) g_{\mu \nu}^{\perp} .
\end{aligned}
$$

Using these relations and eqs. (172)-(178) given in Appendix 3 , we obtain the following results for the $L<J$ 
and $L>J$ states:

$$
\begin{aligned}
& -\int \frac{d \Omega}{4 \pi} S p\left[V_{\mu_{1} \ldots \mu_{J}}^{L<J}\left(m_{1}+\hat{k}_{1}\right) V_{\nu_{1} \ldots \nu_{J}}^{L<J}\left(m_{2}-\hat{k}_{2}\right)\right]= \\
& \left(2\left(s-\delta^{2}\right)-\frac{8 J|\boldsymbol{k}|^{2}}{2 J+1}\right)|\boldsymbol{k}|^{2(J-1)} O_{\nu_{1} \ldots \nu_{J}}^{\mu_{1} \ldots \mu_{J}}(-1)^{J}, \\
& -\int \frac{d \Omega}{4 \pi} S p\left[V_{\mu_{1} \ldots \mu_{J}}^{L>J}\left(m_{1}+\hat{k}_{1}\right) V_{\nu_{1} \ldots \nu_{J}}^{L>J}\left(m_{2}-\hat{k}_{2}\right)\right]= \\
& \quad\left(2\left(s-\delta^{2}\right)-\frac{8(J+1)|\boldsymbol{k}|^{2}}{2 J+1}\right)|\boldsymbol{k}|^{2(J+1)} O_{\nu_{1} \ldots \nu_{J}}^{\mu_{1} \ldots \mu_{J}}(-1)^{J} .
\end{aligned}
$$

In case of spin-1 operators, the two triplet states with the same parity are not orthogonal to each other; the interference loop diagram is equal to

$$
\begin{gathered}
-\int \frac{d \Omega}{4 \pi} S p\left[V_{\mu_{1} \ldots \mu_{J}}^{L<J}\left(m_{1}+\hat{k}_{1}\right) V_{\nu_{1} \ldots \nu_{J}}^{L>J}\left(m_{2}-\hat{k}_{2}\right)\right]= \\
8 \frac{\sqrt{J(J+1)}}{2 J+1}|\boldsymbol{k}|^{2(J+1)} O_{\nu_{1} \ldots \nu_{J}}^{\mu_{1} \ldots \mu_{J}}(-1)^{J}
\end{gathered}
$$

The one-loop diagram for the $L=J$ triplet state is given by

$$
\begin{aligned}
& -\int \frac{d \Omega}{4 \pi} S p\left[V_{\mu_{1} \ldots \mu_{J}}\left(m_{1}+\hat{k}_{1}\right)\left(k^{\perp}\right) V_{\nu_{1} \ldots \nu_{J}}\left(k^{\perp}\right)\left(m_{2}-\hat{k}_{2}\right)\right] \\
& =2\left(s-\delta^{2}\right)|\boldsymbol{k}|^{2 J} O_{\nu_{1} \ldots \nu_{J}}^{\mu_{1} \ldots \mu_{J}}(-1)^{J} .
\end{aligned}
$$

Direct calculations show that the transition loop diagrams between the triplet state with $L=J$ and the triplet states with $L>J$ and $L<J$ vanish. For vertex operators describing pure spin states (66) and (68), one has the following one-loop diagrams:

$$
\begin{aligned}
& -\int \frac{d \Omega}{4 \pi} S p\left[\tilde{V}_{\mu_{1} \ldots \mu_{J}}^{L<J}\left(m_{1}+\hat{k}_{1}\right) \tilde{V}_{\nu_{1} \ldots \nu_{J}}^{L<J}\left(m_{2}-\hat{k}_{2}\right)\right]= \\
& 2\left(s-\delta^{2}\right)|\boldsymbol{k}|^{2(J-1)} O_{\nu_{1} \ldots \nu_{J}}^{\mu_{1} \ldots \mu_{J}}(-1)^{J}, \\
& -\int \frac{d \Omega}{4 \pi} S p\left[\tilde{V}_{\mu_{1} \ldots \mu_{J}}^{L>J}\left(m_{1}+\hat{k}_{1}\right) \tilde{V}_{\nu_{1} \ldots \nu_{J}}^{L>J}\left(m_{2}-\hat{k}_{2}\right)\right]= \\
& 2\left(s-\delta^{2}\right)|\boldsymbol{k}|^{2(J+1)} O_{\nu_{1} \ldots \nu_{J}}^{\mu_{1} \ldots \mu_{J}}(-1)^{J}
\end{aligned}
$$

\subsection{Cross sections}

The expressions for one-loop diagrams can be used to calculate cross sections for different spin-orbital momentum states. The cross section is given by

$$
d \sigma=\frac{(2 \pi)^{4}|A|^{2}}{4|\boldsymbol{q}| \sqrt{s}} d \Phi=\frac{|\boldsymbol{k}|}{|\boldsymbol{q}|} \frac{\rho(s)}{16 \pi s} \int \frac{d \Omega}{4 \pi}|A|^{2} .
$$

To calculate the amplitude squared $|A|^{2}$, one can use the expressions for the one-loop diagram given by eqs. (86), (91), (89) and (90).

For the ${ }^{1} L_{J}$ state, one has

$$
d \sigma=\frac{2 J+1}{64 \pi m^{2}}|\boldsymbol{k}|^{2 J+1}|\boldsymbol{q}|^{2 J-1} s
$$

For the ${ }^{3} L_{J}$ state $(L=J)$, the result is

$$
d \sigma=\frac{2 J+1}{64 \pi m^{2}}|\boldsymbol{k}|^{2 J+1}|\boldsymbol{q}|^{2 J-1} s .
$$

The decay of the ${ }^{3} L_{J}$ states with $(L<J)$ and ${ }^{3} L_{J}(L>J)$ is determined by the sum of two vertices:

$$
A_{t r}^{L \neq J}=\lambda_{1} V_{\mu_{1} \ldots \mu_{J}}^{L<J}+\lambda_{2} V_{\mu_{1} \ldots \mu_{J}}^{L>J}
$$

Then, the cross section is equal to

$$
d \sigma=\lambda_{1}^{2} d \sigma_{11}+\lambda_{2}^{2} d \sigma_{22}+\lambda_{1} \lambda_{2}\left(d \sigma_{12}+d \sigma_{21}\right),
$$

where

$$
\begin{aligned}
d \sigma_{11} & =\frac{2 J+1}{256 \pi s m^{2}}|\boldsymbol{k}|^{2 J-1}|\boldsymbol{q}|^{2 J-3} \\
& \times\left[2 s-\frac{8 J|\boldsymbol{k}|^{2}}{2 J+1}\right]\left[2 s-\frac{8 J|\boldsymbol{q}|^{2}}{2 J+1}\right] \\
d \sigma_{22} & =\frac{2 J+1}{256 \pi s m^{2}}|\boldsymbol{k}|^{2 J+3}|\boldsymbol{q}|^{2 J+1} \\
& \times\left[2 s-\frac{8(J+1)|\boldsymbol{k}|^{2}}{2 J+1}\right]\left[2 s-\frac{8(J+1)|\boldsymbol{q}|^{2}}{2 J+1}\right], \\
d \sigma_{12} & =\frac{1}{4 \pi s m^{2}} \frac{J(J+1)}{2 J+1}|\boldsymbol{k}|^{2 J+3}|\boldsymbol{q}|^{2 J+1} \\
d \sigma_{21} & =\frac{1}{4 \pi s m^{2}} \frac{J(J+1)}{2 J+1}|\boldsymbol{q}|^{2 J+3}|\boldsymbol{k}|^{2 J+1}
\end{aligned}
$$

For pure spin-1 operators, $V_{\mu_{1} \ldots \mu_{J}}^{L<J}$ and $V_{\mu_{1} \ldots \mu_{J}}^{L>J}$, the cross section reads

$$
d \sigma=\lambda_{1}^{2} d \sigma_{11}+\lambda_{2}^{2} d \sigma_{22}
$$

where

$$
\begin{aligned}
& d \sigma_{11}=\frac{(2 J+1) s}{64 \pi m^{2}}|\boldsymbol{k}|^{2 J-1}|\boldsymbol{q}|^{2 J-3} \\
& d \sigma_{22}=\frac{(2 J+1) s}{64 \pi m^{2}}|\boldsymbol{k}|^{2 J+3}|\boldsymbol{q}|^{2 J+1}
\end{aligned}
$$

\section{Decay into $3 / 2^{+}$and $1 / 2^{+}$particles}

Let $k_{1}$ be the momentum of the $3 / 2^{+}$particle and $k_{2}$ the momentum of $1 / 2^{+}$. In this case, there are two spin states, $S=1$ and $S=2$. Let us start from the $S=1$ states. Such states are constructed using the vector spinors $\psi_{\alpha}$ for $3 / 2$ spin particle and spin operators.

For ${ }^{3} L_{J}(J=L-1)$, that corresponds to $\left(0^{-}, 1^{+}, 2^{-}, 3^{+} \ldots\right)$ states, and the operators read

$$
\begin{aligned}
W_{\mu_{1} \ldots \mu_{J}}^{(1)} & =\bar{\psi}_{\alpha}\left(k_{1}\right) V_{\mu_{1} \ldots \mu_{J}}^{(1) \alpha} u\left(-k_{2}\right), \\
V_{\mu_{1} \ldots \mu_{J}}^{(1) \alpha} & =i \gamma_{5} X_{\alpha \mu_{1} \ldots \mu_{J}}^{(J+1)} .
\end{aligned}
$$

For ${ }^{3} L_{J}(J=L+1)$, the operators are given by

$$
\begin{aligned}
W_{\mu_{1} \ldots \mu_{J}}^{(2)} & =\bar{\psi}_{\alpha_{1}}\left(k_{1}\right) i \gamma_{5} X_{\alpha_{2} \ldots \alpha_{J}}^{(J-1)} O_{\mu_{1} \ldots \mu_{J}}^{\alpha_{1} \ldots \alpha_{J}} u\left(-k_{2}\right) \\
V_{\mu_{1} \ldots \mu_{J}}^{(2) \alpha_{1}} & =i \gamma_{5} X_{\alpha_{2} \ldots \alpha_{J}}^{(J-1)} O_{\mu_{1} \ldots \mu_{J}}^{\alpha_{1} \ldots \alpha_{J}}
\end{aligned}
$$


where the projection operator is needed for index symmetrisation. For ${ }^{3} L_{J}(J=L)\left(1^{-}, 2^{+}, 3^{-}, 4^{+} \ldots\right)$, the operators can be expressed as

$$
\begin{aligned}
W_{\mu_{1} \ldots \mu_{J}}^{(3)} & =\gamma_{5} \varepsilon_{\alpha_{1} \beta \xi \eta} \bar{\psi}_{\beta}\left(k_{1}\right) k_{\xi} P_{\eta} X_{\alpha_{2} \ldots \alpha_{J}}^{(J-1)} O_{\mu_{1} \ldots \mu_{J}}^{\alpha_{1} \ldots \alpha_{J}} u\left(-k_{2}\right), \\
V_{\mu_{1} \ldots \mu_{J}}^{(3) \beta} & =\gamma_{5} \varepsilon_{\alpha_{1} \beta \xi \eta} k_{\xi} P_{\eta} X_{\alpha_{2} \ldots \alpha_{J}}^{(J-1)} O_{\mu_{1} \ldots \mu_{J}}^{\alpha_{1} \ldots \alpha_{J}} .
\end{aligned}
$$

In case of $S=2$, there are five operators. For ${ }^{5} L_{J}$ $(J=L+2)$ the operators are given by

$$
\begin{aligned}
W_{\mu_{1} \ldots \mu_{J}}^{(4)} & =\bar{\psi}_{\alpha_{1}}\left(k_{1}\right) \gamma_{\alpha_{2}} O_{\nu_{1} \nu_{2}}^{\alpha_{1} \alpha_{2}} X_{\nu_{3} \ldots \nu_{J}}^{(J-2)} O_{\mu_{1} \ldots \mu_{J}}^{\nu_{1} \ldots \nu_{J}} u\left(-k_{2}\right), \\
V_{\mu_{1} \ldots \mu_{J}}^{(4) \alpha_{1}} & =\gamma_{\alpha_{2}} O_{\nu_{1} \nu_{2}}^{\alpha_{1} \alpha_{2}} X_{\nu_{3} \ldots \nu_{J}}^{(J-2)} O_{\mu_{1} \ldots \mu_{J}}^{\nu_{1} \ldots \nu_{J}}
\end{aligned}
$$

for ${ }^{5} L_{J}(J=L-2)$ by

$$
\begin{aligned}
W_{\mu_{1} \ldots \mu_{J}}^{(5)} & =\bar{\psi}_{\alpha_{1}}\left(k_{1}\right) \gamma_{\alpha_{2}} X_{\alpha_{1} \alpha_{2} \nu_{1} \ldots \nu_{J}}^{(J+2)} u\left(-k_{2}\right), \\
V_{\mu_{1} \ldots \mu_{J}}^{(5) \alpha_{1}} & =\gamma_{\alpha_{2}} X_{\alpha_{1} \alpha_{2} \nu_{1} \ldots \nu_{J}}^{(J+2)},
\end{aligned}
$$

for ${ }^{5} L_{J}(J=L)$ by

$$
\begin{aligned}
W_{\mu_{1} \ldots \mu_{J}}^{(6)} & =\bar{\psi}_{\alpha}\left(k_{1}\right) \gamma_{\beta} O_{\alpha \beta}^{\nu_{1} \xi} X_{\xi \nu_{2} \ldots \nu_{J}}^{(J)} O_{\mu_{1} \ldots \mu_{J}}^{\nu_{1} \ldots \nu_{J}} u\left(-k_{2}\right), \\
V_{\mu_{1} \ldots \mu_{J}}^{(6) \alpha} & =\gamma_{\beta} O_{\alpha \beta}^{\nu_{1} \xi} X_{\xi \nu_{2} \ldots \nu_{J}}^{(J)} O_{\mu_{1} \ldots \mu_{J}}^{\nu_{1} \ldots \nu_{J}}
\end{aligned}
$$

for ${ }^{5} L_{J}(J=L-1)$ by

$$
\begin{aligned}
& W_{\mu_{1} \ldots \mu_{J}}^{(7)}= \\
& i \varepsilon_{\nu_{1} \beta \tau \eta} k_{\tau} P_{\eta} O_{\beta \xi}^{\alpha_{1} \alpha_{2}} \bar{\psi}_{\alpha_{1}}\left(k_{1}\right) \gamma_{\alpha_{2}} X_{\xi \nu_{2} \ldots \nu_{J}}^{(J)} O_{\mu_{1} \ldots \mu_{J}}^{\nu_{1} \ldots \nu_{J}} u\left(-k_{2}\right), \\
& V_{\mu_{1} \ldots \mu_{J}}^{(7) \alpha_{1}}=i \varepsilon_{\nu_{1} \beta \tau \eta} k_{\tau} P_{\eta} O_{\beta \xi}^{\alpha_{1} \alpha_{2}} \gamma_{\alpha_{2}} X_{\xi \nu_{2} \ldots \nu_{J}}^{(J)} O_{\mu_{1} \ldots \mu_{J}}^{\nu_{1} \ldots \nu_{J}},
\end{aligned}
$$

and for ${ }^{5} L_{J}(J=L+1)$ by

$$
\begin{aligned}
& W_{\mu_{1} \ldots \mu_{J}}^{(8)}= \\
& i \varepsilon_{\nu_{1} \beta \tau \eta} k_{\tau} P_{\eta} O_{\beta \nu_{2}}^{\alpha_{1} \alpha_{2}} \bar{\psi}_{\alpha_{1}}\left(k_{1}\right) \gamma_{\alpha_{2}} X_{\nu_{3} \ldots \nu_{J}}^{(J-2)} O_{\mu_{1} \ldots \mu_{J}}^{\nu_{1} \ldots \nu_{J}} u\left(-k_{2}\right), \\
& V_{\mu_{1} \ldots \mu_{J}}^{(8) \alpha_{1}}=i \varepsilon_{\nu_{1} \beta \tau \eta} k_{\tau} P_{\eta} O_{\beta \nu_{2}}^{\alpha_{1} \alpha_{2}} \gamma_{\alpha_{2}} X_{\nu_{3} \ldots \nu_{J}}^{(J-2)} O_{\mu_{1} \ldots \mu_{J}}^{\nu_{1} \ldots \nu_{J}} .
\end{aligned}
$$

The one-loop diagram amplitudes for the corresponding operators are calculated in Appendix 4.

\section{Example: amplitude for the reaction $p p \rightarrow p K^{+} \Lambda$}

Let us start from $p p$ scattering with the production of a resonance $R$ in the intermediate state which decays into $K^{+} \Lambda$. The diagram for the process is shown in Fig. 1 Consider the partial wave amplitude for the $p p$ having quantum numbers $J=n, L$ and $S$ in the initial state. The general form of the angular dependent part of this partial amplitude is

$$
\begin{aligned}
& \left(\bar{u}\left(-k_{1}\right) Q_{\nu_{1} \ldots \nu_{n}}^{(S, L, J)} u\left(k_{2}\right)\right) \bar{u}\left(q_{3}\right) \tilde{N}_{\alpha_{1} \ldots \alpha_{m}}(R \rightarrow K \Lambda) \\
& \times F_{\beta_{1} \ldots \beta_{m}}^{\alpha_{1} \ldots \alpha_{m}}\left(q_{2}+q_{3}\right) Q_{\beta_{1} \ldots \beta_{m} \nu_{1} \ldots \nu_{n}}^{(S, L, J)} u\left(-q_{1}\right) \\
& -\left\{k_{1} \Leftrightarrow k_{2}\right\}
\end{aligned}
$$

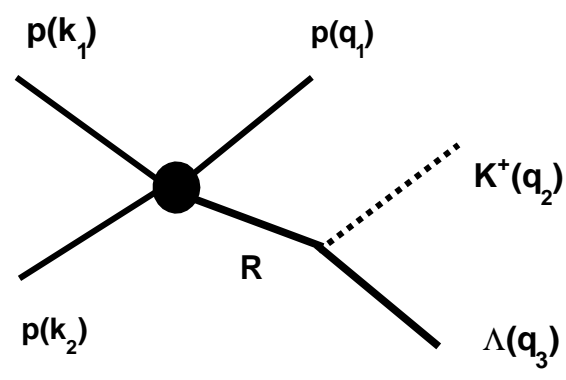

Fig. 1. Reaction $p p \rightarrow p K^{+} \Lambda: p p$ scattering with production of a resonance $R$ in the intermediate state

where $P=q_{1}+q_{2}+q_{3}=k_{1}+k_{2}$. The resonance $R$ with spin $J=m+1 / 2$ is produced in the intermediate state and decays into a final state meson and a nucleon.

The initial $p p$ state operator $Q_{\nu_{1} \ldots \nu_{n}}^{(S, L, J)}$ is defined by Eq. (60) for $S=0$ and eqs. (63), (65), (67) for $S=1$. If the resonance in the intermediate state has the spin $1 / 2(m=0)$, the same expressions define the $R p$ state operator. For the spin-3/2 resonance in the intermediate state, the operator $Q_{\beta_{1} \ldots \beta_{m} \nu_{1} \ldots \nu_{n}}^{(S, L, J}$ is defined by eqs. (100)-(107). The operators for the $R \rightarrow 0^{-}+1 / 2^{+}$transitions were defined in [147:

$$
\begin{array}{ll}
\tilde{N}_{\mu_{1} \ldots \mu_{n}}^{+}=X_{\mu_{1} \ldots \mu_{n}}^{(n)} & 1 / 2^{-}, 3 / 2^{+}, 5 / 2^{-} \ldots \\
\tilde{N}_{\mu_{1} \ldots \mu_{n}}^{-}=i \gamma_{\nu} \gamma_{5} X_{\nu \mu_{1} \ldots \mu_{n}}^{(n+1)} & 1 / 2^{+}, 3 / 2^{-}, 5 / 2^{+} \ldots
\end{array}
$$

Let us write down the amplitude for the $1 / 2^{+}$resonance in the intermediate state. In this case, one finds

$$
\begin{aligned}
& M=\sum_{S, L, J} \bar{u}\left(-k_{1}\right) Q_{\nu_{1} \ldots \nu_{n}}^{(S, L, J)} u\left(k_{2}\right) \bar{u}\left(q_{3}\right) \tilde{N}^{-}\left(q_{23}^{\perp}\right) \\
& \times \frac{\hat{q}_{2}+\hat{q}_{3}+\sqrt{s_{23}}}{2 \sqrt{s_{23}}} B W\left(s_{23}\right) Q_{\nu_{1} \ldots \nu_{n}}^{(S, L, J)} u\left(-q_{1}\right) A^{(S, L, J)}\left(s, s_{23}\right) \\
& -\left\{k_{1} \Leftrightarrow k_{2}\right\}, \\
& q_{23}^{\perp}=\left(q_{2}-q_{3}\right)_{\nu}\left(g_{\mu \nu}-\frac{Q_{23 \mu} Q_{23 \nu}}{s_{23}}\right), \quad Q_{23}=q_{2}+q_{3}
\end{aligned}
$$

where $A^{(S, L, J)}\left(s, s_{23}\right)$ is the partial amplitude for $p p \rightarrow R p$ and $B W\left(s_{23}\right.$ parameterises the resonance $\left.R\right)$.

Another type of processes, which may contribute to this reaction, is the t-channel exchange of pseudoscalar and vector particles $(\pi$ and $\rho$ ). This diagram is shown in Fig. 2,

First, consider the exchange of a $\rho$ meson. The vertex operators for the transition baryon $\rightarrow$ vector meson + baryon are given in [147]. Thus the $p \rho \rightarrow R$ operators are given by

$$
\begin{aligned}
A_{\text {lower }}^{(i-)} & =\bar{u}\left(Q_{23}\right) V^{(i-) \mu}\left(k_{2}^{\perp}\right) u\left(k_{2}\right) \rho_{\mu}, \quad i=1,2, \\
k_{2 \mu}^{\perp} & =\frac{1}{2}\left(k_{2}-k_{t}\right)_{\nu}\left(g_{\mu \nu}-\frac{Q_{23 \mu} Q_{23 \nu}}{s_{23}}\right), \quad \text { (111) }
\end{aligned}
$$

where

$$
V_{\alpha_{1} \ldots \alpha_{n}}^{(1-) \mu}\left(k^{\perp}\right)=\gamma_{\xi} \gamma_{\mu}^{\perp} X_{\xi \alpha_{1} \ldots \alpha_{n}}^{(n+1)}\left(k^{\perp}\right)
$$




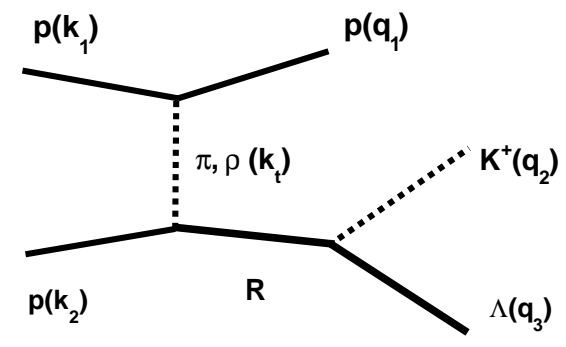

Fig. 2. Reaction $p p \rightarrow p K^{+} \Lambda$ : t-channel exchange diagram.

$$
V_{\alpha_{1} \ldots \alpha_{n}}^{(2-) \mu}\left(k^{\perp}\right)=X_{\mu \alpha_{1} \ldots \alpha_{n}}^{(n+1)}\left(k^{\perp}\right) .
$$

Here, $k_{t}=Q_{23}-k_{2}$ is the $\rho$-meson momentum. In case of a spin- $1 / 2$ resonance in the intermediate state, one should use Eq.(112) with $n=0$. For the upper operator, one has:

$$
\begin{aligned}
A_{u p p e r}^{(i-)} & =\bar{u}\left(q_{1}\right) V^{(i-) \mu}\left(q_{1}^{\perp}\right) u\left(k_{1}\right) \rho_{\mu}, \quad i=1,2 \\
q_{1 \mu}^{\perp} & =\frac{1}{2}\left(q_{1}-k_{t}\right)_{\nu}\left(g_{\mu \nu}-\frac{k_{1 \mu} k_{1 \nu}}{k_{1}^{2}}\right)
\end{aligned}
$$

Summing over the polarisations yields:

$$
\sum_{\text {polarisations }} \rho_{\alpha} \rho_{\beta}=-g_{\alpha \beta}+\frac{k_{t \alpha} k_{t \beta}}{k_{t}^{2}} .
$$

Finally, we arrive at the following amplitude for the $\rho$ exchange:

$$
\begin{aligned}
A_{\rho}^{(i)} & =\bar{u}\left(q_{1}\right) V^{(i-) \mu}\left(q_{1}^{\perp}\right) u\left(k_{1}\right) \bar{u}\left(q_{3}\right) \tilde{N}^{-}\left(q_{23}^{\perp}\right) \\
& \times \frac{\hat{q}_{2}+\hat{q}_{3}+\sqrt{s_{23}}}{2 \sqrt{s_{23}}} B W\left(s_{23}\right) V^{(i-) \nu}\left(k_{2}^{\perp}\right) u\left(k_{2}\right) \\
& \times\left(-g_{\mu \nu}+\frac{k_{t \mu} k_{t \nu}}{k_{t}^{2}}\right), \quad i=1,2 .
\end{aligned}
$$

In case of t-channel exchange of a pseudoscalar meson, $\pi$, one should substitute the operator $V^{(i-) \mu}$ by $\tilde{N}^{-}$, so we have

$$
\begin{aligned}
A_{\pi} & =\bar{u}\left(q_{1}\right) \tilde{N}^{-}\left(q_{1}^{\perp}\right) u\left(k_{1}\right) \bar{u}\left(q_{3}\right) \tilde{N}^{-}\left(q_{23}^{\perp}\right) \\
& \times \frac{\hat{q}_{2}+\hat{q}_{3}+\sqrt{s_{23}}}{2 \sqrt{s_{23}}} B W\left(s_{23}\right) \tilde{N}^{-}\left(k_{2}^{\perp}\right) u\left(k_{2}\right) .
\end{aligned}
$$

\section{Triangle-diagram amplitude with pion-nucleon rescattering: logarithmic singularity}

In the amplitudes describing production of three-particle final states, the unitarity condition is fulfilled automatically when final-state rescattering is properly taken into account. However, rescattering may lead to singularities where the amplitude tends to infinity. The triangle diagram with the $\Delta$ in the intermediate state gives us an example of this type of the process: it has logarithmic singularity which under certain conditions $\left(\sqrt{s} \sim m_{N}+m_{\Delta}\right)$ can be near the physical region.
Because of $\sqrt{s} \sim m_{N}+m_{\Delta}$, we consider the amplitude $p p \rightarrow N \Delta$ with $L^{\prime}=0$ (the produced $N \Delta$ system is in the $S$-wave). The quantum numbers of the final state are then restricted to

$$
J^{P}=1^{+}, 2^{+} \text {. }
$$

The initial $p p$ system $(I=1)$ has

$$
\begin{array}{ll}
S=0: & L=0,2,4, \ldots \quad J^{P}=0^{+}, 2^{+}, 4^{+}, \ldots \\
S=1: & L=1,3,5, \ldots \quad J^{P}=0^{-}, 1^{-}, 2^{-}, 3^{-}, \ldots
\end{array}
$$

We thus consider the transition

$$
p p\left(S=0, L=2, J^{P}=2^{+}\right) \rightarrow N \Delta\left(S^{\prime}=2, L^{\prime}=0, J^{P}=2^{+}\right) \text {. }
$$

The corresponding pole amplitude reads:

$$
\begin{aligned}
& A_{N N \rightarrow N N \pi}^{p o l e}=C_{N N \rightarrow N N \pi}^{p o l e} G_{p p \rightarrow N \Delta}^{\left(S=0, S^{\prime}=2, L=2, L^{\prime}=0, J=2\right)}(s) \\
& \times\left(\bar{u}\left(p_{1}^{\prime}\right) g_{\Delta} k_{1 \mu}^{\prime \perp p_{\Delta}} \frac{\Delta_{\mu \nu}\left(p_{\Delta}\right)}{m_{\Delta}^{2}-p_{\Delta}^{2}-i m_{\Delta} \Gamma_{\Delta}} \gamma_{\nu^{\prime}} u\left(-p_{2}^{\prime}\right)\right) \\
& \times\left(\bar{u}\left(-p_{2}\right) i \gamma_{5} X_{\nu \nu^{\prime}}^{(2)}(k) u\left(p_{1}\right)\right) .
\end{aligned}
$$

Here, the factor $C_{N N \rightarrow N N \pi}^{\text {pole }}$ refers to the isotopic ClebschGordan coefficients, and

$$
k_{1 \mu}^{\prime \perp p} p_{\Delta}=g_{\mu \mu^{\prime}}^{\perp p_{\Delta}} p_{1 \mu^{\prime}}^{\prime} .
$$

The numerator of the $3 / 2$-spin fermion propagator is written in the form used in [146, 147]:

$$
\begin{aligned}
\Delta_{\mu \nu}(k) & =\frac{\hat{k}+M_{\Delta}}{2 M_{\Delta}}\left(-g_{\mu \nu}^{\perp}+\frac{1}{3} \gamma_{\mu}^{\perp} \gamma_{\nu}^{\perp}\right), \\
\gamma_{\mu}^{\perp} & =g_{\mu \nu}^{\perp} \gamma_{\nu}, \quad g_{\mu \nu}^{\perp}=g_{\mu \nu}-\frac{k_{\mu} k_{\nu}}{M_{\Delta}^{2}} .
\end{aligned}
$$

The decay vertex $g_{\Delta}$ is determined by the imaginary part of the loop diagram $\Delta \rightarrow N \pi \rightarrow \Delta$. For the sake of simplicity, we change in (119): $\Gamma_{\nu^{\prime}}\left(k_{\perp}^{\prime}\right) \rightarrow \gamma_{\nu^{\prime}}$; however, using definition (59) one can easily rewrite Eq. (119) in a more rigid form.

Taking into account the rescattering process in the amplitude (119), $\pi N \rightarrow \Delta \rightarrow \pi N$, one has the following triangle-diagram amplitude (see Fig. 3):

$$
\begin{aligned}
& A_{N N \rightarrow N N \pi}^{\text {triangle }}=C_{N N \rightarrow N N \pi}^{\text {triangle }} G_{p p \rightarrow N \Delta}^{\left(S=0, S^{\prime}=2, L=2, L^{\prime}=0, J=2\right)}(s) \\
& \times\left(\overline { u } ( p _ { 1 } ^ { \prime } ) \left[\int \frac{d^{4} k_{\pi}}{i(2 \pi)^{4}} \frac{1}{m_{\pi}^{2}-k_{\pi}^{2}-i 0}\right.\right. \\
& \times g_{\Delta} k_{1 \mu^{\prime}}^{\prime \perp p_{\Delta}^{\prime}} \frac{\Delta_{\mu^{\prime} \nu}\left(p_{\Delta}^{\prime}\right)}{m_{\Delta}^{2}-p_{\Delta}^{\prime 2}-i m_{\Delta} \Gamma_{\Delta}} \gamma_{\nu^{\prime}} \frac{-\hat{p}_{2}^{\prime \prime}+m}{m^{2}-p_{2}^{\prime \prime 2}-i 0} \\
& \left.\left.\times g_{\Delta} k_{2 \mu^{\prime \prime}}^{\prime \prime \perp} p_{\Delta}^{\prime \prime} \frac{\Delta_{\mu^{\prime \prime} \nu^{\prime \prime}}\left(-p_{\Delta}\right)}{m_{\Delta}^{2}-p_{\Delta}^{2}-i m_{\Delta} \Gamma_{\Delta}} g_{\Delta} k_{2 \nu^{\prime \prime}}^{\prime \perp p}\right] u\left(-p_{2}^{\prime}\right)\right) \\
& \times\left(\bar{u}\left(-p_{2}\right) i \gamma_{5} X_{\nu \nu^{\prime}}^{(2)}(k) u\left(p_{1}\right)\right) .
\end{aligned}
$$

Here,

$$
\begin{aligned}
& k_{1 \mu^{\prime}}^{\perp \perp p_{\Delta}^{\prime}}=g_{\mu^{\prime} \alpha}^{\perp p_{\Delta}^{\prime}} p_{1 \alpha}^{\prime}, \quad k_{2 \mu^{\prime \prime}}^{\prime \perp} p_{\Delta}^{\prime \prime}=g_{\mu^{\prime \prime} \alpha}^{\perp p_{\Delta}} p_{2 \alpha}^{\prime \prime}, \\
& k_{2 \nu^{\prime \prime}}^{\perp \perp p_{\Delta}}=g_{\nu^{\prime \prime} \alpha}^{\perp p_{\Delta}} p_{2 \alpha}^{\prime},
\end{aligned}
$$


and

$$
\begin{aligned}
p_{\Delta}^{\prime} & =p_{1}^{\prime}+k_{\pi}, \quad p_{\Delta}=p_{2}^{\prime}+p_{\pi}=p_{2}^{\prime \prime}+k_{\pi}, \\
P & =p_{\Delta}^{\prime}+p_{2}^{\prime \prime} .
\end{aligned}
$$

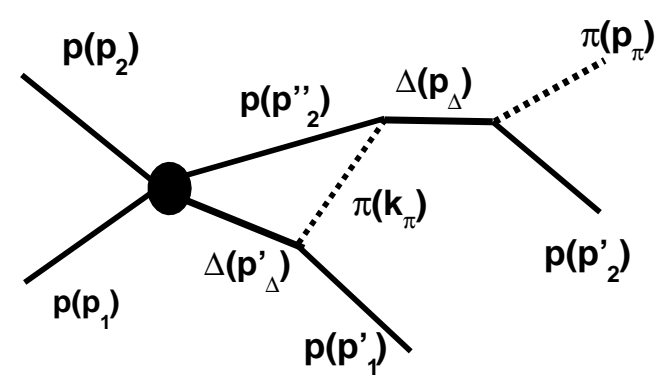

Fig. 3. Triangle diagram with final state pion-nucleon rescattering

One can simplify (122) by extracting the numerator in the singular point that corresponds to

$$
m_{\Delta}^{2}=p_{\Delta}^{\prime 2}, \quad m^{2}=p_{2}^{\prime \prime 2}, \quad m_{\pi}^{2}=k_{\pi}^{2} .
$$

Then, Eq. (122) reads:

$$
\begin{aligned}
& A_{N N \rightarrow N N \pi}^{\text {triangle }}=C_{N N \rightarrow N N \pi}^{\text {triangle }} G_{p p \rightarrow N \Delta}^{\left(S=0, S^{\prime}=2, L=2, L^{\prime}=0, J=2\right)}(s) \\
& \times\left(\bar{u}\left(p_{1}^{\prime}\right) g_{\Delta} k_{1 \mu^{\prime}}^{\perp \perp p_{\Delta}^{\prime}}(\operatorname{tr}) \Delta_{\mu^{\prime} \nu}\left(p_{\Delta}^{\prime}(\operatorname{tr})\right) \gamma_{\nu^{\prime}}\left(-\hat{p}_{2}^{\prime \prime}(\operatorname{tr})+m\right) g_{\Delta}\right. \\
& \left.\times k_{2 \mu^{\prime \prime}}^{\prime \prime \perp} p_{\Delta}^{\prime \prime}(\operatorname{tr}) \frac{\Delta_{\mu^{\prime \prime} \nu^{\prime \prime}}\left(-p_{\Delta}\right)}{m_{\Delta}^{2}-p_{\Delta}^{2}-i m_{\Delta} \Gamma_{\Delta}} g_{\Delta} k_{2 \nu^{\prime \prime}}^{\perp \perp p} u\left(-p_{2}^{\prime}\right)\right) \\
& \times\left(\bar{u}\left(-p_{2}\right) i \gamma_{5} X_{\nu \nu^{\prime}}^{(2)}(k) u\left(p_{1}\right)\right) \\
& \times \int \frac{d^{4} k_{\pi}}{i(2 \pi)^{4}} \frac{1}{m_{\pi}^{2}-k_{\pi}^{2}-i 0} \frac{1}{m^{2}-\left(p_{\Delta}-k_{\pi}\right)^{2}-i 0} \\
& \times \frac{1}{m_{\Delta}^{2}-\left(P-p_{\Delta}+k_{\pi}\right)^{2}-i m_{\Delta} \Gamma_{\Delta}}
\end{aligned}
$$

The momenta $k_{1 \mu^{\prime}}^{\perp \perp p_{\Delta}^{\prime}}(\operatorname{tr}), p_{\Delta}^{\prime}(\operatorname{tr}), p_{2}^{\prime \prime}(\operatorname{tr}), k_{2 \mu^{\prime \prime}}^{\prime \prime} p_{\Delta}^{\prime \prime}(\operatorname{tr})$ obey the constraints (125). The integral in (126) corresponds to the triangle diagram with spinless particles. Its calculation is performed in Appendix 5.

\section{Box-diagram singularities in the reaction $N N \rightarrow \Delta \Delta \rightarrow N N \pi \pi$}

The primary aim of a partial wave analysis is to extract the pole singularities of amplitudes, thus determining resonances. Of course, the existence of other singularities like threshold singularities should be taken into account. This is possible using the $K$-matrix technique, see 150 , 151,152 and references therein. Singularities due to resonances in the intermediate state need more sophisticated treatment.
The existence of triangle-diagram singularities, which may be located near the physical region of a three-particle production reaction, was proven in [153, 154]: these singularities diverge as $\ln \left(s-s_{0}\right)$. Stronger singularities (with a $\left(s-s_{0}\right)^{-1 / 2}$ behaviour) are related to box diagrams [155, 156 .

Here, we present box-diagram and triangle-diagram singular amplitudes for the reaction $N N \rightarrow \Delta \Delta \rightarrow N N \pi \pi$ taking into account the spin structure in a way which allows us to include these singular amplitudes into partial wave analyses (this was not yet done in [155, 156]).

Let us introduce the following notations for the twopole and box diagrams in the reactions $N N \rightarrow \Delta \Delta \rightarrow$ $N N \pi \pi$ (see Figs. 4 and 5).

The initial state momenta are:

$$
P_{1}+P_{2}=P, \quad P^{2}=W^{2}, \quad \frac{1}{2}\left(P_{1}-P_{2}\right)=q
$$

Final state momenta:

$$
\begin{aligned}
& \left(p_{1}+p_{3}\right)^{2}=s_{13}, \quad\left(p_{1}+p_{3}+p_{2}\right)^{2}=s_{4}, \\
& p_{1}+p_{3}=k_{1}, \quad k_{1}^{\perp}=\frac{1}{2}\left(p_{1}-p_{3}\right)^{\perp k_{1}}=p_{1}^{\perp k_{1}}=-p_{3}^{\perp k_{1}}, \\
& \left(p_{2}+p_{4}\right)^{2}=s_{24}, \quad\left(p_{2}+p_{4}+p_{1}\right)^{2}=s_{1}, \\
& p_{2}+p_{4}=k_{2}, \quad k_{2}^{\perp}=\frac{1}{2}\left(p_{2}-p_{4}\right)^{\perp k_{2}}=p_{2}^{\perp k_{2}}=-p_{4}^{\perp k_{2}}, \\
& \left(p_{1}+p_{2}\right)^{2}=s, \quad p_{1}+p_{2}=p .
\end{aligned}
$$

Here, the symbol $\perp k_{i}$ means the component of a vector perpendicular to $k_{i}$ :

$$
p_{\mu}^{\perp k_{i}}=p_{\mu}-k_{i \mu} \frac{\left(k_{i} p\right)}{k_{i}^{2}} .
$$

\section{$9.1(N N)_{S-w a v e}$ state with $J^{P}=0^{+}$, two-pole diagram}

In $p p$ collision with $I=1$, the $S$-wave $\Delta \Delta$ state is produced. First, consider the two-pole diagram of Fig. 4 . The

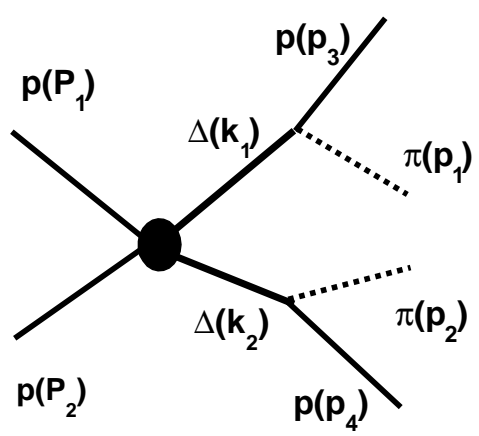

Fig. 4. Pole diagram for reaction $N N \rightarrow \Delta \Delta \rightarrow N N \pi \pi$

amplitude for the production and decay of two $\Delta$-isobars, 
$N N \rightarrow \Delta \Delta \rightarrow N N \pi \pi$, omitting charge indices and corresponding Clebsch-Gordan coefficients, reads:

$$
\begin{aligned}
& A_{N N \rightarrow \Delta \Delta \rightarrow(N \pi)(N \pi)}=\left(\bar{u}\left(-P_{2}\right) u\left(P_{1}\right)\right) G_{N N \rightarrow \Delta \Delta}( \\
& \times\left(\bar{u}\left(p_{3}\right) g_{\Delta} k_{1 \mu}^{\perp} \frac{\Delta_{\mu \nu^{\prime}}\left(k_{1}\right)}{M_{\Delta}^{2}-s_{13}-i M_{\Delta} \Gamma_{\Delta}}\right. \\
& \left.\times \frac{\Delta_{\nu^{\prime} \nu}\left(-k_{2}\right)}{M_{\Delta}^{2}-s_{24}-i M_{\Delta} \Gamma_{\Delta}}(-) k_{2 \nu}^{\perp} g_{\Delta} u\left(-p_{4}\right)\right) .
\end{aligned}
$$

\subsection{Box-diagram amplitude with pion-pion rescattering}

The box-diagram amplitude with pion-pion rescattering in the Feynman technique (see Fig. [5) is equal to

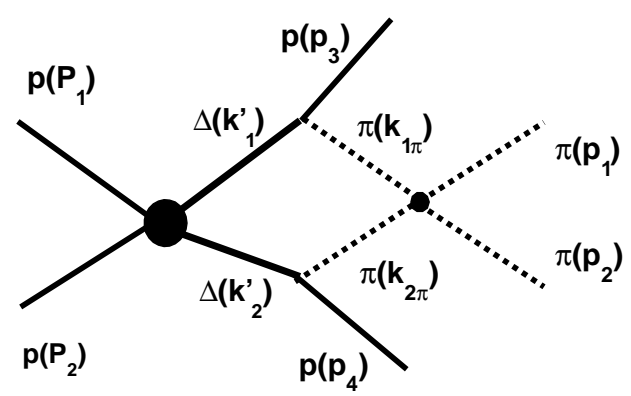

Fig. 5. Box-diagram with pion-pion rescattering

$$
\begin{aligned}
& A_{N N \rightarrow \Delta \Delta \rightarrow N N+(\pi \pi \rightarrow \pi \pi)_{S}}=A_{\pi \pi \rightarrow \pi \pi}^{S-\text { wave }}(s) \\
\times & G_{N N \rightarrow \Delta \Delta}(W)\left(\bar{u}\left(-P_{2}\right) u\left(P_{1}\right)\right)\left(\overline { u } ( p _ { 3 } ) \left[\int \frac{d^{4} k^{\prime}}{i(2 \pi)^{4}}\right.\right. \\
\times & \frac{g_{\Delta} k_{1 \mu}^{\prime \perp} \Delta_{\mu \nu^{\prime}}\left(k_{1}^{\prime}\right) \Delta_{\nu^{\prime} \nu}\left(-k_{2}^{\prime}\right)(-) k_{2 \nu}^{\prime \perp} g_{\Delta}}{\left(M_{\Delta}^{2}-s_{13}^{\prime}-i M_{\Delta} \Gamma_{\Delta}\right)\left(M_{\Delta}^{2}-s_{24}^{\prime}-i M_{\Delta} \Gamma_{\Delta}\right)} \\
\times & \left.\left.\frac{1}{\left(m_{\pi}^{2}-k_{1 \pi}^{2}-i 0\right)\left(m_{\pi}^{2}-k_{2 \pi}^{2}-i 0\right)}\right] u\left(-p_{4}\right)\right) .
\end{aligned}
$$

The factor $A_{\pi \pi \rightarrow \pi \pi}^{S-\text { wave }}(s)$ is the $S$-wave $\pi \pi$-scattering amplitude. Here we take into account the low-energy $\pi \pi$ interaction only. In the $K$-matrix representation, it is written in the form

$$
A_{\pi \pi \rightarrow \pi \pi}^{S-\text { wave }}(s)=\frac{K(s)}{1-i \rho(s) K(s)}, \quad \rho(s)=\frac{1}{16 \pi} \sqrt{\frac{s-4 m_{\pi}^{2}}{s}} .
$$

In (132), we take into account the full $S$-wave as observed experimentally, including the so-called sigma-meson, independently of its existence. Generally speaking, it is possible to account for higher waves as well, but the box diagram with two $\Delta$ 's leads to singularities near the physical region of the production process at $\sqrt{s} \lesssim 0.6 \mathrm{GeV}$ only.

The approximation used in the calculation of the box diagram (131) is related to the extraction of the leading terms of the singular amplitude. To this aim, we fix the numerator of the integrand in the propagator poles by setting

$$
k_{1}^{\prime 2} \rightarrow M_{\Delta}^{2}, k_{2}^{\prime 2} \rightarrow M_{\Delta}^{2}, k_{1 \pi}^{2} \rightarrow m_{\pi}^{2}, k_{2 \pi}^{2} \rightarrow m_{\pi}^{2},
$$

which leads in (131) to the substitution

$$
\begin{aligned}
& k_{1 \mu}^{\prime \perp} \rightarrow k_{1 \mu}^{\perp}(\text { box })=-p_{3}^{\perp k_{1}(\text { box })}, k_{1}^{\prime} \rightarrow k_{1}(\text { box }), \\
& \left.k_{2 \nu}^{\prime \perp} \rightarrow k_{2 \nu}^{\perp}(\text { box })=-p_{4}^{\perp k_{2}(\text { box })}, k_{2}^{\prime} \rightarrow k_{2}(\text { box })\right) .
\end{aligned}
$$

Now, in the c.m. system, the momenta $k_{a}$ (box) read

$$
\begin{aligned}
& k_{1}(\mathrm{box})=\left(W / 2,0,0, \sqrt{W^{2} / 4-M_{\Delta}^{2}}\right), \\
& k_{2}(\mathrm{box})=\left(W / 2,0,0,-\sqrt{W^{2} / 4-M_{\Delta}^{2}}\right) .
\end{aligned}
$$

Here, we denote the four-momentum as $k=\left(k_{0}, k_{x}, k_{y}, k_{z}\right)$. Under the constraints of Eq. (133), the numerator of the integrand does not depend on the integration variables, and it can be written separately for the leading singular $(L S)$ term:

$$
\begin{aligned}
& A_{N N \rightarrow \Delta \Delta \rightarrow N N+(\pi \pi \rightarrow \pi \pi)_{S}}^{(L S)}=A_{\pi \pi \rightarrow \pi \pi}^{S-\text { wave }}(s) G_{N N \rightarrow \Delta \Delta}(W) \\
& \times\left(\bar{u}\left(-P_{2}\right) u\left(P_{1}\right)\right)\left(\bar{u}\left(p_{3}\right) g_{\Delta}\left(-p_{3 \mu}^{\perp k_{1}(\text { box })}\right) \Delta_{\mu \nu^{\prime}}\left(k_{1}(\text { box })\right)\right. \\
& \left.\times \Delta_{\nu^{\prime} \nu}\left(-k_{2}(\text { box })\right) p_{4 \nu}^{\perp k \text { (box) }} g_{\Delta} u\left(-p_{4}\right)\right) \\
& \times \int \frac{d^{4} k^{\prime}}{i(2 \pi)^{4}} \frac{1}{\left(M_{\Delta}^{2}-\left(\frac{1}{2} p+k^{\prime}+p_{3}\right)^{2}-i M_{\Delta} \Gamma_{\Delta}\right)} \\
& \times \frac{1}{\left(M_{\Delta}^{2}-\left(\frac{1}{2} p-k^{\prime}+p_{4}\right)^{2}-i M_{\Delta} \Gamma_{\Delta}\right)} \\
& \times \frac{1}{\left(m_{\pi}^{2}-\left(\frac{1}{2} p+k^{\prime}\right)^{2}-i 0\right)\left(m_{\pi}^{2}-\left(\frac{1}{2} p-k^{\prime}\right)^{2}-i 0\right)},(136
\end{aligned}
$$

where

$$
\frac{1}{2} p+k^{\prime}=k_{1 \pi}, \quad \frac{1}{2} p-k^{\prime}=k_{2 \pi}, \quad p_{1}+p_{2}=p .
$$

The box-diagram integral of Eq. (136) is calculated in Appendix 6: in this Appendix, we demonstrate the effects of the box diagram on the $\pi \pi$ spectra.

\subsection{Box-diagram amplitude with pion-nucleon rescattering}

In the Feynman technique, the box-diagram amplitude with pion-nucleon rescattering in the resonance state $(I=$ $3 / 2, J=3 / 2$ ) reads (see Fig. 6):

$$
\begin{aligned}
& A_{N N \rightarrow \Delta \Delta \rightarrow N \pi+(N \pi \rightarrow N \pi)_{\Delta}}=G_{N N \rightarrow \Delta \Delta}(W) \\
& \times\left(\bar{u}\left(-P_{2}\right) u\left(P_{1}\right)\right) \\
& \times\left(\bar{u}\left(p_{3}\right) g_{\Delta} \frac{1}{2}\left(p_{2}-p_{3}\right)_{\mu}^{\perp p_{\Delta}} \frac{\Delta_{\mu \mu^{\prime}}\left(p_{\Delta}\right)}{M_{\Delta}^{2}-p_{\Delta}^{2}-i M_{\Delta} \Gamma_{\Delta}}\right. \\
& \times\left[\int \frac{d^{4} k^{\prime}}{i(2 \pi)^{4}} \frac{1}{2}\left(k_{2 \pi}^{\prime}-k_{1 N}^{\prime}\right)_{\mu^{\prime}}^{\perp p_{\Delta}} g_{\Delta} \frac{\hat{k}_{1 N}^{\prime}+m_{N}}{m_{N}^{2}-k_{1 N}^{\prime 2}-i 0} g_{\Delta}\right.
\end{aligned}
$$




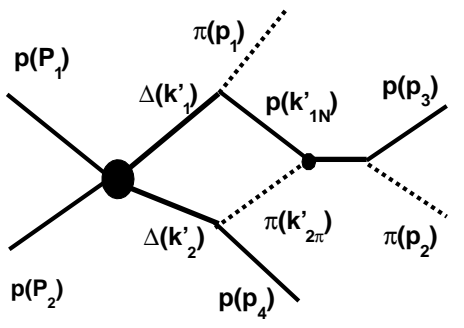

Fig. 6. Box-diagram with pion-nucleon rescattering

$$
\begin{aligned}
& \times \frac{\frac{1}{2}\left(p_{1}-k_{1 N}^{\prime}\right)_{\mu^{\prime}}^{\perp k_{1}^{\prime}} \Delta_{\mu^{\prime} \nu^{\prime}}\left(k_{1}^{\prime}\right) \Delta_{\nu^{\prime} \nu}\left(-k_{2}^{\prime}\right) \frac{1}{2}\left(-k_{2 \pi}^{\prime}+p_{4}\right)_{\nu}^{\perp k_{2}^{\prime}}}{\left(M_{\Delta}^{2}-k_{1}^{\prime 2}-i M_{\Delta} \Gamma_{\Delta}\right)\left(M_{\Delta}^{2}-k_{2}^{\prime 2}-i M_{\Delta} \Gamma_{\Delta}\right)} \\
& \left.\left.\times \frac{1}{\left(m_{\pi}^{2}-k_{2 \pi}^{\prime 2}-i 0\right)}\right] g_{\Delta} u\left(-p_{4}\right)\right],
\end{aligned}
$$

where $p_{\Delta}=p_{2}+p_{3}$. By fixing the numerator of (138) at

$$
k_{1}^{\prime 2} \rightarrow M_{\Delta}^{2}, k_{2}^{\prime 2} \rightarrow M_{\Delta}^{2}, k_{1 \pi}^{2} \rightarrow m_{\pi}^{2}, k_{1 N}^{2} \rightarrow m_{N}^{2},
$$

we write the leading singular $(L S)$ terms of the box-diagram amplitude as follows:

$$
\begin{aligned}
& A_{N N \rightarrow \Delta \Delta \rightarrow N \pi+(N \pi \rightarrow N \pi)_{\Delta}}^{(L S)}=G_{N N \rightarrow \Delta \Delta}(W) \\
& \times\left(\bar{u}\left(-P_{2}\right) u\left(P_{1}\right)\right) \\
& \times\left(\bar{u}\left(p_{3}\right) g_{\Delta} \frac{1}{2}\left(p_{2}-p_{3}\right)_{\mu}^{\perp p_{\Delta}} \frac{\Delta_{\mu \mu^{\prime}}\left(p_{\Delta}\right)}{M_{\Delta}^{2}-p_{\Delta}^{2}-i M_{\Delta} \Gamma_{\Delta}}\right. \\
& \times \frac{1}{2}\left(k_{1}(\mathrm{box})-p_{1}-k_{2}(\mathrm{box})+p_{4}\right)_{\mu^{\prime}}^{\perp p_{\Delta}} \\
& \times g_{\Delta}\left(\hat{k}_{1}(\mathrm{box})-\hat{p}_{1}+m_{N}\right) g_{\Delta} p_{1 \mu^{\prime}}^{\perp k_{1}(\mathrm{box})} \\
& \left.\times \Delta_{\mu^{\prime} \nu^{\prime}}\left(k_{1}(\mathrm{box})\right) \Delta_{\nu^{\prime} \nu}\left(-k_{2}(\mathrm{box})\right) p_{4 \nu}^{\perp k_{2}(\mathrm{box})} g_{\Delta} u\left(-p_{4}\right)\right) \\
& \times \int \frac{d^{4} k_{\pi}}{i(2 \pi)^{4}} \frac{1}{\left(m_{N}^{2}-\left(p_{\Delta}-k_{\pi}^{2}\right)^{2}-i 0\right)} \\
& \times \frac{1}{\left(M_{\Delta}^{2}-\left(p_{\Delta}-k_{\pi}+p_{1}\right)^{2}-i M_{\Delta} \Gamma_{\Delta}\right)} \\
& \times \frac{1}{\left(M_{\Delta}^{2}-\left(k_{\pi}+p_{4}\right)^{2}-i M_{\Delta} \Gamma_{\Delta}\right)\left(m_{\pi}^{2}-k_{\pi}^{2}-i 0\right)} . \quad(140
\end{aligned}
$$

\section{$9.4(N N)_{D-w a v e}$ state with $J^{P}=2^{+}$, two-pole and box diagrams}

The production of $\Delta \Delta$ near the threshold in the S-wave leads to a $J^{P}=2^{+}$state as well and, correspondingly, to a strong box-diagram singularity in this wave. In the $J^{P}=$ $2^{+}$wave, the transition $(N N)_{D-\text { wave }} \rightarrow(\Delta \Delta)_{S-\text { wave }}$ is related to the two-pole amplitude

$$
\begin{aligned}
& A_{(N N)_{D} \rightarrow(\Delta \Delta)_{S} \rightarrow(N \pi)(N \pi)}=G_{N N \rightarrow \Delta \Delta}(W) \\
& \times\left(\bar{u}\left(-P_{2}\right) X_{\nu^{\prime} \nu^{\prime \prime}}^{(2)}(q) u\left(P_{1}\right)\right) \\
& \times\left(\bar{u}\left(p_{3}\right) g_{\Delta} k_{1 \mu}^{\perp} \frac{\Delta_{\mu \nu^{\prime}}\left(k_{1}\right)}{M_{\Delta}^{2}-s_{13}-i M_{\Delta} \Gamma_{\Delta}}\right. \\
& \left.\times \frac{\Delta_{\nu^{\prime \prime} \nu}\left(-k_{2}\right)}{M_{\Delta}^{2}-s_{24}-i M_{\Delta} \Gamma_{\Delta}}\left(-k_{2 \nu}^{\perp}\right) g_{\Delta} u\left(-p_{4}\right)\right) .
\end{aligned}
$$

The box-diagram amplitude with the pion-pion rescattering is given by

$$
\begin{aligned}
& A_{N N \rightarrow \Delta \Delta \rightarrow N N+(\pi \pi \rightarrow \pi \pi)_{S}}^{(L S)}=A_{\pi \pi \rightarrow \pi \pi}^{S-\text { wave }}(s) G_{N N \rightarrow \Delta \Delta}(W) \\
& \times\left(\bar{u}\left(-P_{2}\right) X_{\nu^{\prime} \nu^{\prime \prime}}^{(2)}(q) u\left(P_{1}\right)\right) \\
& \times\left(\bar{u}\left(p_{3}\right) g_{\Delta}\left(-p_{3 \mu}^{\perp k_{1}(\mathrm{box})}\right) \Delta_{\mu \nu^{\prime}}\left(k_{1}(\mathrm{box})\right)\right. \\
& \left.\quad \times \Delta_{\nu^{\prime \prime} \nu}\left(-k_{2}(\text { box })\right) p_{4 \nu}^{\perp k(\text { box })} g_{\Delta} u\left(-p_{4}\right)\right) \\
& \times \int \frac{d^{4} k^{\prime}}{i(2 \pi)^{4}} \frac{1}{\left(M_{\Delta}^{2}-\left(\frac{1}{2} p+k^{\prime}+p_{3}\right)^{2}-i M_{\Delta} \Gamma_{\Delta}\right)} \\
& \times \frac{1}{\left(M_{\Delta}^{2}-\left(\frac{1}{2} p-k^{\prime}+p_{4}\right)^{2}-i M_{\Delta} \Gamma_{\Delta}\right)} \\
& \times \frac{1}{\left(m_{\pi}^{2}-\left(\frac{1}{2} p+k^{\prime}\right)^{2}-i 0\right)\left(m_{\pi}^{2}-\left(\frac{1}{2} p-k^{\prime}\right)^{2}-i 0\right)} \cdot(142
\end{aligned}
$$

In the leading singular-term approach, the box-diagram amplitude with the pion-nucleon rescattering can be written in the form

$$
\begin{aligned}
& A_{N N \rightarrow \Delta \Delta \rightarrow N \pi+(N \pi \rightarrow N \pi)_{\Delta}}^{(L S)}=G_{N N \rightarrow \Delta \Delta}(W) \\
& \times\left(\bar{u}\left(-P_{2}\right) X_{\nu^{\prime} \nu^{\prime \prime}}^{(2)}(q) u\left(P_{1}\right)\right) \\
& \times\left(\bar{u}\left(p_{3}\right) g_{\Delta} \frac{1}{2}\left(p_{2}-p_{3}\right)_{\mu}^{\perp p_{\Delta}} \frac{\Delta_{\mu \mu^{\prime}}\left(p_{\Delta}\right)}{M_{\Delta}^{2}-p_{\Delta}^{2}-i M_{\Delta} \Gamma_{\Delta}}\right. \\
& \times \frac{1}{2}\left(k_{1}(\mathrm{box})-p_{1}-k_{2}(\mathrm{box})+p_{4}\right)_{\mu^{\prime}}^{\perp p_{\Delta}} g_{\Delta} \\
& \times\left(\hat{k}_{1}(\mathrm{box})-\hat{p}_{1}+m_{N}\right) g_{\Delta} p_{1 \mu^{\prime}}^{\perp k_{1}(\mathrm{box})} \Delta_{\mu^{\prime} \nu^{\prime}}\left(k_{1}(\mathrm{box})\right) \\
& \left.\times \Delta_{\nu^{\prime \prime} \nu}\left(-k_{2}(\mathrm{box})\right) p_{4 \nu}^{\perp k_{2}(\mathrm{box})} g_{\Delta} u\left(-p_{4}\right)\right) \\
& \times \int \frac{d^{4} k_{\pi}}{i(2 \pi)^{4}} \frac{1}{\left(m_{N}^{2}-\left(p_{\Delta}-k_{\pi}^{2}\right)^{2}-i 0\right)} \\
& \times \frac{1}{\left(M_{\Delta}^{2}-\left(p_{\Delta}-k_{\pi}+p_{1}\right)^{2}-i M_{\Delta} \Gamma_{\Delta}\right)} \\
& \times \frac{1}{\left(M_{\Delta}^{2}-\left(k_{\pi}+p_{4}\right)^{2}-i M_{\Delta} \Gamma_{\Delta}\right)\left(m_{\pi}^{2}-k_{\pi}^{2}-i 0\right)} .
\end{aligned}
$$

\section{Conclusion}

We have developed a new method for the partial wave analysis of data on the baryon-baryon and baryon-antibaryon collision. The method is based on the operator decomposition approach which was successfully applied before to a number of meson-induced reactions. The article emphasises the analysis of reactions with three or four particles in the final state, where triangle and box singularities might play an important role. A full set of partial wave amplitudes is constructed for nucleon-nucleon elastic scattering and for $N \Delta$ and $\Delta \Delta$ production. With these amplitudes, expressions for partial widths and for reaction cross sections are presented. Some examples how to calculate contributions from triangle and box diagrams in simple cases are explicitly given. The application of the 
methods developed here to the analysis of new data obtained and expected from COSY should provide valuable information about the hadron spectrum and properties of hadron interaction.

\section{Acknowledgments}

We would like to thank L.G. Dakhno for helpful discussions and critical reading of the manuscript. The work was supported by a FFE grant of the Research Center Jülich and by the Deutsche Forschungsgemeinschaft within the Sonderforschungsbereich SFB/TR16. We would like to thank the Alexander von Humboldt foundation for generous support in the initial phase of the project, A.V.A. for a AvH fellowship and A.V.S. for the Friedrich-Wilhelm Bessel award. A. Sarantsev gratefully acknowledges the support from Russian Science Support Foundation. This work is also supported by Russian Foundation for Basic Research and RSGSS 5788.2006.2 (Russian State Grant Scientific School).

\section{Appendix 1}

The baryon wave functions $\psi(p)$ and $\bar{\psi}(p)=\psi^{+}(p) \gamma_{0}$ obey the Dirac equation

$$
(\hat{p}-m) \psi(p)=0, \quad \bar{\psi}(p)(\hat{p}-m)=0 .
$$

The $\gamma$-matrices were used in the form

$$
\begin{aligned}
& \gamma_{0}=\left(\begin{array}{cc}
I & 0 \\
0 & -I
\end{array}\right), \quad \gamma=\left(\begin{array}{cc}
0 & \boldsymbol{\sigma} \\
-\boldsymbol{\sigma} & 0
\end{array}\right), \\
& \gamma_{5}=i \gamma_{0} \gamma_{1} \gamma_{2} \gamma_{3}=\left(\begin{array}{ll}
0 & I \\
I & 0
\end{array}\right), \\
& \gamma_{0}^{+}=\gamma_{0}, \quad \gamma^{+}=-\gamma,
\end{aligned}
$$

with the standard Pauli matrices

$$
\begin{aligned}
& \sigma_{1}=\left(\begin{array}{ll}
0 & 1 \\
1 & 0
\end{array}\right), \sigma_{2}=\left(\begin{array}{cc}
0 & -i \\
i & 0
\end{array}\right), \sigma_{3}=\left(\begin{array}{cc}
1 & 0 \\
0 & -1
\end{array}\right), \\
& \sigma_{a} \sigma_{b}=i \varepsilon_{a b c} \sigma_{c} .
\end{aligned}
$$

The Dirac equation gives four wave functions

$$
\begin{aligned}
& j=1,2: \psi_{j}(p)=\sqrt{p_{0}+m}\left(\begin{array}{c}
\varphi_{j} \\
\frac{(\boldsymbol{\sigma} \boldsymbol{p})}{p_{0}+m} \varphi_{j}
\end{array}\right), \\
& \bar{\psi}_{j}(p)=\sqrt{p_{0}+m}\left(\varphi_{j}^{+},-\varphi_{j}^{+} \frac{(\boldsymbol{\sigma} \boldsymbol{p})}{p_{0}+m}\right) \\
& j=3,4: \quad \psi_{j}(-p)=i \sqrt{p_{0}+m}\left(\begin{array}{c}
\frac{(\boldsymbol{\sigma} \boldsymbol{p})}{p_{0}+m} \chi_{j} \\
\chi_{j}
\end{array}\right), \\
& \bar{\psi}_{j}(-p)=-i \sqrt{p_{0}+m}\left(\chi_{j}^{+} \frac{(\boldsymbol{\sigma} \boldsymbol{p})}{p_{0}+m},-\chi_{j}^{+}\right),
\end{aligned}
$$

where $\varphi_{j}$ and $\chi_{j}$ are two-component spinors

$$
\varphi_{j}=\left(\begin{array}{c}
\varphi_{j 1} \\
\varphi_{j 2}
\end{array}\right), \quad \chi_{j}=\left(\begin{array}{c}
\chi_{j 1} \\
\chi_{j 2}
\end{array}\right)
$$

which are normalized as follows:

$$
\varphi_{j}^{+} \varphi_{\ell}=\delta_{j \ell}, \quad \chi_{j}^{+} \chi_{\ell}=\delta_{j \ell} .
$$

The solutions with $j=3,4$ refer to antibaryons. The corresponding wave function is given by

$$
j=3,4: \quad \psi_{j}^{c}(p)=C \bar{\psi}_{j}^{T}(-p),
$$

where

$$
C=\gamma_{2} \gamma_{0}=\left(\begin{array}{cc}
0 & -\sigma_{2} \\
-\sigma_{2} & 0
\end{array}\right)=\left(\begin{array}{cccc}
0 & 0 & 0 & i \\
0 & 0 & -i & 0 \\
0 & i & 0 & 0 \\
-i & 0 & 0 & 0
\end{array}\right)
$$

The equation (151) reads:

$$
\begin{aligned}
& j=3,4 \text { : }
\end{aligned}
$$

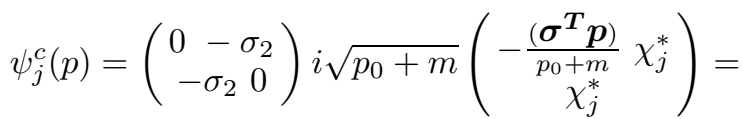

$$
\begin{aligned}
& -i \sqrt{p_{0}+m}\left(\begin{array}{c}
\sigma_{2} \chi_{j}^{*} \\
\frac{(\boldsymbol{\sigma} \boldsymbol{p})}{p_{0}+m} \sigma_{2} \chi_{j}^{*}
\end{array}\right)=\sqrt{p_{0}+m}\left(\begin{array}{c}
\boldsymbol{\varphi}_{j}^{c} \\
\frac{(\boldsymbol{\sigma} \boldsymbol{p})}{p_{0}+m} \varphi_{j}^{c}
\end{array}\right) .
\end{aligned}
$$

In (153), we have used the commutator

$$
\sigma_{2}\left(\boldsymbol{\sigma}^{\boldsymbol{T}} \boldsymbol{p}\right)=-\sigma_{1} p_{1} \sigma_{2}-\sigma_{2} p_{2} \sigma_{2}-\sigma_{3} p_{3} \sigma_{2}=-(\boldsymbol{\sigma} \boldsymbol{p}) \sigma_{2} .
$$

We define the two-component spinor for antibaryons as

$$
\varphi_{j}^{c}=-i \sigma_{2} \chi_{j}^{*}=\left(\begin{array}{cc}
0 & -1 \\
1 & 0
\end{array}\right) \chi_{j}^{*}=\left(\begin{array}{c}
-\chi_{j 2}^{*} \\
\chi_{j \ell}^{*}
\end{array}\right) .
$$

The wave functions defined in eqs. (147)-(148) are normalized as follows:

$$
\begin{array}{ll}
j, \ell=1,2: & \left(\bar{\psi}_{j}(p) \psi_{\ell}(p)\right)=2 m \delta_{j \ell}, \\
j, \ell=3,4: & \left(\bar{\psi}_{j}(-p) \psi_{\ell}(-p)\right)=-2 m \delta_{j \ell} .
\end{array}
$$

They obey the completeness relation

$$
\begin{aligned}
& \sum_{j=1,2} \psi_{j \alpha}(p) \bar{\psi}_{j \beta}(p)=(\hat{p}+m)_{\alpha \beta}, \\
& \sum_{j=3,4} \psi_{j \alpha}(-p) \bar{\psi}_{j \beta}(-p)=(\hat{p}-m)_{\alpha \beta} .
\end{aligned}
$$

\section{Appendix 2}

(i) The $S$-wave terms in the the nonrelativistic limit.

We consider the operators with $L=0$ from eqs. (60) and (66) in the c.m. system $\left(\mathbf{p}_{1}=-\mathbf{p}_{2}=\mathbf{k}\right.$ and $\mathbf{p}^{\prime}{ }_{1}=-\mathbf{p}_{2}^{\prime}=$ $\left.\mathbf{k}^{\prime}\right)$. For $L=0$, we have the following operators in the nonrelativistic approach:

$$
\begin{gathered}
Q^{000}(k)=i \gamma_{5}=i\left(\begin{array}{cc}
0 & I \\
I & 0
\end{array}\right), \\
Q^{101}(k)=\Gamma_{\mu} \simeq\left(\begin{array}{cc}
0 & \sigma \\
-\sigma & 0
\end{array}\right) .
\end{gathered}
$$


In the c.m. system, we have:

$$
\begin{aligned}
& j, j^{\prime}=1,2: \psi_{N j}\left(p_{1}\right) \simeq \sqrt{2 m_{N}}\left(\begin{array}{c}
\varphi_{N j} \\
\frac{(\boldsymbol{\sigma} \boldsymbol{k})}{2 m_{N}} \varphi_{N j}
\end{array}\right), \\
& \bar{\psi}_{N j^{\prime}}\left(p_{1}^{\prime}\right) \simeq \sqrt{2 m_{N}}\left(\varphi_{N j^{\prime}}^{+},-\varphi_{N j^{\prime}}^{+} \frac{\left(\boldsymbol{\sigma} \boldsymbol{k}^{\prime}\right)}{2 m_{N}}\right), \\
& \ell, \ell^{\prime}=3,4: \psi_{\Lambda \ell^{\prime}}^{c}\left(-p_{2}^{\prime}\right) \simeq i \sqrt{2 m_{\Lambda}}\left(\begin{array}{c}
\frac{-\left(\boldsymbol{\sigma} \boldsymbol{k}^{\prime}\right)}{2 m_{\Lambda}} \chi_{\Lambda \ell^{\prime}}^{c} \\
\chi_{\Lambda \ell^{\prime}}^{c}
\end{array}\right) \text {, } \\
& \bar{\psi}_{\Lambda \ell}^{c}\left(-p_{2}\right) \simeq-i \sqrt{2 m_{\Lambda}}\left(\chi_{\Lambda \ell}^{c+} \frac{-(\boldsymbol{\sigma} \boldsymbol{k})}{2 m_{\Lambda}},-\chi_{\Lambda \ell}^{c+}\right),
\end{aligned}
$$

where $\varphi_{N j}$ and $\chi_{\Lambda \ell}^{c}$ are two-component spinors. For the waves with $J=0,1$ we have

$$
\begin{aligned}
L= & 0, J=0: \quad\left(\bar{\psi}_{N}\left(p_{1}^{\prime}\right) \hat{Q}^{000}\left(k^{\prime}\right) \psi_{\Lambda}^{c}\left(-p_{2}^{\prime}\right)\right) \\
& \times\left(\bar{\psi}_{\Lambda}^{c}\left(-p_{2}\right) \hat{Q}^{000}(k) \psi_{N}\left(p_{1}\right)\right) A_{N \Lambda \rightarrow N \Lambda}^{(0,00,0)}(s) \simeq \\
& \sqrt{4 m_{N} m_{\Lambda}}\left(\varphi_{N j^{\prime}}^{+} \chi_{\Lambda \ell^{\prime}}^{c}\right)\left(\chi_{\Lambda \ell}^{c+} \varphi_{N j}\right) \sqrt{4 m_{N} m_{\Lambda}} \\
& \times A_{N \Lambda \rightarrow N \Lambda}^{(0,00,0)}(s) \\
L= & 0, J=1: \quad\left(\bar{\psi}_{N}\left(p_{1}^{\prime}\right) \hat{Q}_{\mu}^{101}\left(k^{\prime}\right) \psi_{\Lambda}^{c}\left(-p_{2}^{\prime}\right)\right) \\
& \times\left(\bar{\psi}_{\Lambda}^{c}\left(-p_{2}\right) \hat{Q}_{\mu}^{101}(k) \psi_{N}\left(p_{1}\right)\right) A_{N \Lambda \rightarrow N \Lambda}^{(1,00,1)}(s) \simeq \\
& i \sqrt{4 m_{N} m_{\Lambda}}\left(\varphi_{N j^{\prime}}^{+} \boldsymbol{\sigma} \chi_{\Lambda \ell^{\prime}}^{c}\right)\left(\chi_{\Lambda \ell}^{c+} \boldsymbol{\sigma} \varphi_{j}\right) i \sqrt{4 m_{N} m_{\Lambda}} \\
& \times A_{N \Lambda \rightarrow N \Lambda}^{(1,00,1)}(s) .
\end{aligned}
$$

Let us consider bispinors with real components. For nucleons, we write

$$
\varphi_{N j}=\left(\begin{array}{c}
\varphi_{\uparrow}(N j) \\
\varphi_{\downarrow}(N j)
\end{array}\right), \varphi_{N j}^{+}=\left(\varphi_{\uparrow}(N j), \varphi_{\downarrow}(N j)\right) .
$$

For the $\Lambda$, we determine the bispinor to be given by

$$
\chi_{\Lambda \ell}^{c}=i \sigma_{2}\left(\begin{array}{c}
\varphi_{\uparrow}(\Lambda \ell) \\
\varphi_{\downarrow}(\Lambda \ell)
\end{array}\right)=\left(\begin{array}{c}
\varphi_{\downarrow}(\Lambda \ell) \\
-\varphi_{\uparrow}(\Lambda \ell)
\end{array}\right)
$$

Within this definition, we can re-write (160) in terms of the traditional technique which uses the Clebsch-Gordan coefficients. For $J=0$, we have

$$
\begin{aligned}
&\left(\chi_{\Lambda \ell}^{c+} \frac{I}{\sqrt{2}} \varphi_{N j}\right)=\left(\varphi_{N j}^{+} \frac{I}{\sqrt{2}} \chi_{\Lambda \ell}^{c}\right)= \\
& \frac{1}{\sqrt{2}}\left(\varphi_{\uparrow}(N j) \varphi_{\downarrow}(\Lambda \ell)-\varphi_{\downarrow}(N j) \varphi_{\uparrow}(\Lambda \ell)\right)= \\
&= \sum_{\alpha} C_{1 / 2 \alpha}^{00}, 1 / 2-\alpha \\
& \varphi_{\alpha}(N j) \varphi_{-\alpha}(\Lambda \ell),
\end{aligned}
$$

and for $J=1, J_{3}=0$,

$$
\begin{aligned}
& \left(\chi_{\Lambda \ell}^{c+} \frac{\sigma_{3}}{\sqrt{2}} \varphi_{N j}\right)=\left(\varphi_{N j}^{+} \frac{\sigma_{3}}{\sqrt{2}} \chi_{\Lambda \ell}^{c}\right)= \\
& \frac{1}{\sqrt{2}}\left(\varphi_{\uparrow}(N j) \varphi_{\downarrow}(\Lambda \ell)+\varphi_{\downarrow}(N j) \varphi_{\uparrow}(\Lambda \ell)\right)= \\
& \sum_{\alpha} C_{1 / 2 \alpha, 1 / 2-\alpha}^{10} \varphi_{\alpha}(N j) \varphi_{-\alpha}(\Lambda \ell) .
\end{aligned}
$$

(ii) The $D$-wave component in the operator $\gamma_{\mu}^{\perp}$.

Equations (159) and (160) allow one to see easily the existence of the $D$-wave admixture in the operator $\gamma_{\mu}^{\perp}$. By using the operator $\hat{Q}_{\mu}^{101}(k)=\gamma_{\mu}^{\perp}$ in (160), one has the following next-to-leading term in the $(J=1)$-wave:

$$
\begin{aligned}
& -\sqrt{4 m_{N} m_{\Lambda}}\left(\varphi_{N j^{\prime}}^{+} \frac{\left(\boldsymbol{\sigma} \boldsymbol{k}^{\prime}\right)}{2 m_{N}} \boldsymbol{\sigma} \frac{\left(\boldsymbol{\sigma} \boldsymbol{k}^{\prime}\right)}{2 m_{\Lambda}} \chi_{\Lambda \ell^{\prime}}^{c}\right) \\
& \times\left(\chi_{\Lambda \ell}^{c+} \frac{(\boldsymbol{\sigma} \boldsymbol{k})}{2 m_{\Lambda}} \boldsymbol{\sigma} \frac{(\boldsymbol{\sigma} \boldsymbol{k})}{2 m_{N}} \varphi_{N j}\right) \sqrt{4 m_{N} m_{\Lambda}} A_{N \Lambda \rightarrow N \Lambda}^{(1,00,1)}(s) .
\end{aligned}
$$

The spin operators in (165) can be presented as

$$
\frac{(\boldsymbol{\sigma} \boldsymbol{k})}{2 m_{\Lambda}} \boldsymbol{\sigma} \frac{(\boldsymbol{\sigma} \boldsymbol{k})}{2 m_{N}} \simeq \frac{\boldsymbol{k}(\boldsymbol{\sigma} \boldsymbol{k})}{2 m_{\Lambda} m_{N}}+\boldsymbol{\sigma} O\left(\frac{\boldsymbol{k}^{2}}{m_{\Lambda} m_{N}}\right),
$$

where the first term in the r.-h. side refers to the $D$-wave, while the second one gives the correction to the $S$-wave term. In the operator $\Gamma_{\alpha}\left(k_{\perp}\right)$, the $D$-wave admixture is canceled due to the second term: $-\left[4 s k_{\perp \alpha}\left(k_{\perp} \gamma\right)\right] /\left[\left(m_{N}+\right.\right.$ $\left.\left.m_{\Lambda}\right)\left(\sqrt{s}+m_{N}+m_{\Lambda}\right)\left(s-\left(m_{N}-m_{\Lambda}\right)^{2}\right)\right]$

\section{Appendix 3. Useful relations for $Z_{\mu_{1} \ldots \mu_{n}}^{\alpha}$ $X_{\nu_{2} \ldots \nu_{n}}^{(n-1)}$} and

In this appendix, we list a few useful expressions.

$$
\begin{aligned}
& Z_{\mu_{1} \ldots \mu_{n}}^{\alpha}=X_{\nu_{2} \ldots \nu_{n}}^{(n-1)} O_{\mu_{1} \ldots \mu_{n}}^{\alpha \nu_{2} \ldots \nu_{n}} \frac{2 n-1}{n}, \\
& Z_{\mu_{1} \ldots \mu_{n}}^{\alpha}(q)(-1)^{n} O_{\nu_{1} \ldots \nu_{n}}^{\mu_{1} \ldots \mu_{n}} Z_{\nu_{1} \ldots \nu_{n}}^{\beta}(k)=\frac{\alpha_{n}}{n^{2}}(-1)^{n} \\
& \times\left(\sqrt{k_{\perp}^{2}} \sqrt{q_{\perp}^{2}}\right)^{n-1}\left[g_{\alpha \beta}^{\perp} P_{n}^{\prime}-\left(\frac{q_{\alpha}^{\perp} q_{\beta}^{\perp}}{q_{\perp}^{2}}+\frac{k_{\alpha}^{\perp} k_{\beta}^{\perp}}{k_{\perp}^{2}}\right) P_{n-1}^{\prime \prime}\right. \\
& \left.+\frac{q_{\alpha}^{\perp} k_{\beta}^{\perp}}{\sqrt{k_{\perp}^{2}} \sqrt{q_{\perp}^{2}}}\left(P_{n-2}^{\prime \prime}-2 P_{n-1}^{\prime}\right)+\frac{k_{\alpha}^{\perp} q_{\beta}^{\perp}}{\sqrt{k_{\perp}^{2}} \sqrt{q_{\perp}^{2}}} P_{n}^{\prime \prime}\right], \\
& X_{\alpha \mu_{1} \ldots \mu_{n}}(q)(-1)^{n} O_{\nu_{1} \ldots \nu_{n}}^{\mu_{1} \ldots \mu_{n}} X_{\beta \nu_{1} \ldots \nu_{n}}(k)=\frac{\alpha_{n}}{(n+1)^{2}}(-1)^{n} \\
& \times\left(\sqrt{k_{\perp}^{2}} \sqrt{q_{\perp}^{2}}\right)^{n+1}\left[g_{\alpha \beta}^{\perp} P_{n+1}^{\prime}-\left(\frac{q_{\alpha}^{\perp} q_{\beta}^{\perp}}{q_{\perp}^{2}}+\frac{k_{\alpha}^{\perp} k_{\beta}^{\perp}}{k_{\perp}^{2}}\right) P_{n+1}^{\prime \prime}\right. \\
& \left.+\frac{q_{\alpha}^{\perp} k_{\beta}^{\perp}}{\sqrt{k_{\perp}^{2}} \sqrt{q_{\perp}^{2}}}\left(P_{n+2}^{\prime \prime}-2 P_{n+1}^{\prime}\right)+\frac{k_{\alpha}^{\perp} q_{\beta}^{\perp}}{\sqrt{k_{\perp}^{2}} \sqrt{q_{\perp}^{2}}} P_{n}^{\prime \prime}\right], \\
& Z_{\mu_{1} \ldots \mu_{n}}^{\alpha}(q)(-1)^{n} O_{\nu_{1} \ldots \nu_{n}}^{\mu_{1} \ldots \mu_{n}} X_{\beta \nu_{1} \ldots \nu_{n}}(k)=\frac{\alpha_{n-1}}{n(n+1)}(-1)^{n} \\
& \times\left(-k_{\perp}^{2}\right)\left(\sqrt{k_{\perp}^{2}} \sqrt{q_{\perp}^{2}}\right)^{n+1}\left[g_{\alpha \beta}^{\perp} P_{n}^{\prime}-\frac{q_{\alpha}^{\perp} q_{\beta}^{\perp}}{q_{\perp}^{2}} P_{n-1}^{\prime \prime}\right. \\
& \left.-\frac{k_{\alpha}^{\perp} k_{\beta}^{\perp}}{k_{\perp}^{2}} P_{n+1}^{\prime \prime}+\frac{q_{\alpha}^{\perp} k_{\beta}^{\perp}}{\sqrt{k_{\perp}^{2}} \sqrt{q_{\perp}^{2}}} P_{n}^{\prime \prime}+\frac{k_{\alpha}^{\perp} q_{\beta}^{\perp}}{\sqrt{k_{\perp}^{2}} \sqrt{q_{\perp}^{2}}} P_{n}^{\prime \prime}\right] \text {. }
\end{aligned}
$$

We now consider some further expressions used in the one-loop diagram calculations. In our case, the operators are constructed of $X_{\alpha \mu_{1} \ldots \mu_{n}}^{(n+1)}$ and $Z_{\mu_{1} \ldots \mu_{n}}^{\beta}$, where $\alpha$ and $\beta$ 
indices to be convoluted with tensors. Let us start with the loop diagram with a $Z$-operator:

$$
\int \frac{d \Omega}{4 \pi} Z_{\mu_{1} \ldots \mu_{n}}^{\alpha}\left(k^{\perp}\right) T_{\alpha \beta} Z_{\nu_{1} \ldots \nu_{n}}^{\beta}\left(k^{\perp}\right)=\lambda O_{\nu_{1} \ldots \nu_{n}}^{\mu_{1} \ldots \mu_{n}}(-1)^{n} \text {. }
$$

For different tensors $T_{\alpha \beta}$, one has the following $\lambda$ 's:

$$
\begin{array}{rlrl}
T_{\alpha \beta} & =g_{\alpha \beta}, & \lambda & =-\frac{\alpha_{n}}{n}|\boldsymbol{k}|^{2 n-2}, \\
T_{\alpha \beta}=k_{\alpha}^{\perp} k_{\beta}^{\perp}, & \lambda & =\frac{\alpha_{n}}{2 n+1}|\boldsymbol{k}|^{2 n} .
\end{array}
$$

Equation (172) can be easily obtained using eqs. (167) and (50), while Eq. (173) can be obtained using eqs. (42) and (50). For the $X$ operators, one has

$$
\int \frac{d \Omega}{4 \pi} X_{\alpha \mu_{1} \ldots \mu_{n}}^{(n+1)}\left(k^{\perp}\right) T_{\alpha \beta} X_{\beta \nu_{1} \ldots \nu_{n}}^{(n+1)}\left(k^{\perp}\right)=\lambda O_{\nu_{1} \ldots \nu_{n}}^{\mu_{1} \ldots \mu_{n}}(-1)^{n},
$$

where

$$
\begin{aligned}
T_{\alpha \beta} & =g_{\alpha \beta}, & \lambda & =-\frac{\alpha_{n}}{n+1}|\boldsymbol{k}|^{2 n+2}, \\
T_{\alpha \beta} & =k_{\alpha}^{\perp} k_{\beta}^{\perp}, & \lambda & =\frac{\alpha_{n}}{2 n+1}|\boldsymbol{k}|^{2 n+4} .
\end{aligned}
$$

To derive Eq. (174), the properties

$$
O_{\alpha \nu_{1} \ldots \nu_{n}}^{\alpha \mu_{1} \ldots \mu_{n}}=\frac{2 n+3}{2 n+1} O_{\nu_{1} \ldots \nu_{n}}^{\mu_{1} \ldots \mu_{n}}
$$

of the projection operator and Eq. (43) are used. The interference term between $X$ and $Z$ operators is given by

$$
\int \frac{d \Omega}{4 \pi} X_{\alpha \mu_{1} \ldots \mu_{n}}^{(n+1)}\left(k^{\perp}\right) T_{\alpha \beta} Z_{\nu_{1} \ldots \nu_{n}}^{\beta}\left(k^{\perp}\right)=\lambda O_{\nu_{1} \ldots \nu_{n}}^{\mu_{1} \ldots \mu_{n}}(-1)^{n},
$$

with

$$
\begin{aligned}
T_{\alpha \beta}=g_{\alpha \beta}, & \lambda=0, \\
T_{\alpha \beta}=k_{\alpha}^{\perp} k_{\beta}^{\perp}, & \lambda=-\frac{\alpha_{n}}{2 n+1}|\boldsymbol{k}|^{2 n+2} .
\end{aligned}
$$

Eq. (178) is calculated using Eq. (167) and the orthogonality properties (44) of the $X$ operators.

\section{Appendix 4. $N \Delta$ one-loop diagrams}

The calculation of the one-loop diagram for different vertex operators is an important step in the construction of the unitary $N \Delta$ amplitude. Consider the loop diagram for $S=1$ and derive all expressions in case of different particle masses ( $m_{1}$ is mass of $\Delta$ and $m_{2}$ is nucleon mass).

Let us start with ${ }^{3} L_{J}(J=L-1)$ states. Using the expression

$$
\begin{aligned}
& S p\left[i \gamma_{5}\left(m_{1}+\hat{k}_{1}\right)\left(g_{\alpha \beta}^{\perp k_{1}}-\frac{\gamma_{\alpha}^{\perp k_{1}} \gamma_{\beta}^{\perp k_{1}}}{3}\right) i \gamma_{5}\left(m_{2}-\hat{k}_{2}\right)\right] \\
& =-\frac{4}{3}\left(g_{\alpha \beta}-\frac{k_{\alpha}^{\perp} k_{\beta}^{\perp}}{m_{1}^{2}}\right)\left(s-\delta^{2}\right),
\end{aligned}
$$

where $\delta=m_{1}-m_{2}$, the one-loop diagram for the operator (100) is given by

$$
\begin{aligned}
& \int \frac{d \Omega}{4 \pi} S p {\left[V_{\mu_{1} \ldots \mu_{n}}^{(1) \alpha}\left(m_{1}+\hat{k}_{1}\right)\left(g_{\alpha \beta}^{\perp k_{1}}-\frac{\gamma_{\alpha}^{\perp k_{1}} \gamma_{\beta}^{\perp k_{1}}}{3}\right)\right.} \\
& \times\left.\times V_{\nu_{1} \ldots \nu_{n}}^{(1) \beta}\left(m_{2}-\hat{k}_{2}\right)\right]= \\
& \frac{4}{3}\left(s-\delta^{2}\right) \frac{\alpha_{n}}{n+1}\left(1+\frac{|\boldsymbol{k}|^{2}(n+1)}{m_{1}^{2}(2 n+1)}\right)|\boldsymbol{k}|^{2 n+2} \\
& \times O_{\nu_{1} \ldots \nu_{n}}^{\mu_{1} \ldots \mu_{n}}(-1)^{n} .
\end{aligned}
$$

Here, eqs. (174) and (175) were used.

For ${ }^{3} L_{J}(J=L+1)$ states, one has

$$
\begin{aligned}
& \int \frac{d \Omega}{4 \pi} S p\left[V_{\mu_{1} \ldots \mu_{n}}^{(2) \alpha}\left(m_{1}+\hat{k}_{1}\right)\left(g_{\alpha \beta}^{\perp k_{1}}-\frac{\gamma_{\alpha}^{\perp k_{1}} \gamma_{\beta}^{\perp k_{1}}}{3}\right)\right. \\
& \left.\times V_{\nu_{1} \ldots \nu_{n}}^{(2) \beta}\left(m_{2}-\hat{k}_{2}\right)\right]= \\
& \frac{4}{3}\left(s-\delta^{2}\right) \frac{\alpha_{n-1}}{2 n-1}\left(1+\frac{|\boldsymbol{k}|^{2} n}{m_{1}^{2}(2 n+1)}\right)|\boldsymbol{k}|^{2 n-2} \\
& \times O_{\nu_{1} \ldots \nu_{n}}^{\mu_{1} \ldots \mu_{n}}(-1)^{n} .
\end{aligned}
$$

Direct calculations also show that transition loop diagrams between ${ }^{3} L_{J}(J=L-1)$ and ${ }^{3} L_{J}(J=L+1)$ states are equal to

$$
\begin{aligned}
& \int \frac{d \Omega}{4 \pi} S p {\left[V_{\mu_{1} \ldots \mu_{n}}^{(1) \alpha}\left(m_{1}+\hat{k}_{1}\right)\left(g_{\alpha \beta}^{\perp k_{1}}-\frac{\gamma_{\alpha}^{\perp k_{1}} \gamma_{\beta}^{\perp k_{1}}}{3}\right)\right.} \\
& \times\left.V_{\nu_{1} \ldots \nu_{n}}^{(2) \beta}\left(m_{2}-\hat{k}_{2}\right)\right]= \\
& \frac{4}{3}\left(s-\delta^{2}\right) \frac{\alpha_{n-1}}{2 n+1} \frac{|\boldsymbol{k}|^{2 n+2}}{m_{1}^{2}} O_{\nu_{1} \ldots \nu_{n}}^{\mu_{1} \ldots \mu_{n}}(-1)^{n+1} .
\end{aligned}
$$

One can also introduce the pure spin operator in a way that the transition loop diagram is equal to zero. Then eqs.(100, 102) can be rewritten in the following way:

$$
W_{\mu_{1} \ldots \mu_{n}}^{(i)}=\bar{\psi}_{\alpha}\left(k_{1}\right) \Gamma_{\alpha \beta}^{3 / 2} V_{\mu_{1} \ldots \mu_{n}}^{(i) \beta} u\left(-k_{2}\right), \quad i=1,2,
$$

where

$$
\Gamma_{\alpha \beta}^{3 / 2}=g_{\alpha \beta}+\frac{4 s k_{\alpha}^{\perp} k_{\beta}^{\perp}}{(s+M \delta)(\sqrt{s}+M)(\sqrt{s}+\delta)} .
$$

Then, it is easy to find that

$$
\begin{aligned}
& S p\left[i \gamma_{5}\left(m_{1}+\hat{k}_{1}\right) \Gamma_{\alpha \alpha^{\prime}}^{3 / 2}\left(g_{\alpha^{\prime} \beta^{\prime}}^{\perp k_{1}}-\frac{\gamma_{\alpha^{\prime}}^{\perp k_{1}} \gamma_{\beta^{\prime}}^{\perp k_{1}}}{3}\right) \Gamma_{\beta \beta^{\prime}}^{3 / 2}\right. \\
& \left.\quad \times i \gamma_{5}\left(m_{2}-\hat{k}_{2}\right)\right]=-\frac{4}{3} g_{\alpha \beta}\left(s-\delta^{2}\right) .
\end{aligned}
$$

Thus, the transition loop diagram vanishes identically.

For ${ }^{3} L_{J}(J=L)$ states, one has:

$$
\begin{aligned}
& \int \frac{d \Omega}{4 \pi} S p {\left[V_{\mu_{1} \ldots \mu_{n}}^{(3) \alpha}\left(m_{1}+\hat{k}_{1}\right)\left(g_{\alpha \beta}^{\perp k_{1}}-\frac{\gamma_{\alpha}^{\perp k_{1}} \gamma_{\beta}^{\perp k_{1}}}{3}\right)\right.} \\
& \times\left.V_{\nu_{1} \ldots \nu_{n}}^{(3) \beta}\left(m_{2}-\hat{k}_{2}\right)\right]= \\
& \frac{4}{3}\left(s-\delta^{2}\right) s \alpha_{n-1} \frac{n+1}{4 n^{2}-1}|\boldsymbol{k}|^{2 n} O_{\nu_{1} \ldots \nu_{n}}^{\mu_{1} \ldots \mu_{n}}(-1)^{n} .
\end{aligned}
$$


To calculate loop diagrams with $S=2$, the following expression is used:

$$
\begin{aligned}
O_{\mu_{1} \mu_{2}}^{\alpha_{1} \alpha_{2}} S p & {\left[\gamma_{\mu_{1}}\left(m_{1}+\hat{k}_{1}\right)\left(g_{\alpha \beta}^{\perp k_{1}}-\frac{\gamma_{\alpha}^{\perp k_{1}} \gamma_{\beta}^{\perp k_{1}}}{3}\right)\right.} \\
& \left.\times \gamma_{\nu_{2}}\left(m_{2}-\hat{k}_{2}\right)\right] O_{\beta_{1} \beta_{2}}^{\nu_{1} \nu_{2}}= \\
a_{1} O_{\beta_{1} \beta_{2}}^{\alpha_{1} \alpha_{2}} & +a_{2} Z_{\alpha_{1} \alpha_{2}}^{\xi} Z_{\beta_{1} \beta_{2}}^{\xi}+a_{3} X_{\alpha_{1} \alpha_{2}}^{(2)} X_{\beta_{1} \beta_{2}}^{(2)},
\end{aligned}
$$

where

$$
\begin{aligned}
& a_{1}=2\left(s-\delta^{2}\right), a_{2}=\frac{32 \delta}{9 m_{1}}-\frac{16}{27 m_{1}^{2}}\left(s-\left(m_{1}+m_{2}\right)^{2}\right), \\
& a_{3}=-\frac{64}{27 m_{1}^{2}} .
\end{aligned}
$$

For ${ }^{5} L_{J}(J=L+2)$, the operator one-loop diagram is equal to

$$
\begin{aligned}
& \int \frac{d \Omega}{4 \pi} S p\left[V_{\mu_{1} \ldots \mu_{n}}^{(4) \alpha}\left(m_{1}+\hat{k}_{1}\right)\left(g_{\alpha \beta}^{\perp k_{1}}-\frac{\gamma_{\alpha}^{\perp k_{1}} \gamma_{\beta}^{\perp k_{1}}}{3}\right)\right. \\
& \left.\times V_{\nu_{1} \ldots \nu_{n}}^{(4) \beta}\left(m_{2}-\hat{k}_{2}\right)\right]= \\
& \frac{\alpha_{n-2}}{2 n-3}|\boldsymbol{k}|^{2 n-4}(-1)^{n} O_{\nu_{1} \ldots \nu_{n}}^{\mu_{1} \ldots \mu_{n}} \\
& \times\left(a_{1}+\frac{9}{4} \frac{n-1}{2 n-1}\left(-a_{2}|\boldsymbol{k}|^{2}+a_{3} \frac{n}{2 n+1}|\boldsymbol{k}|^{4}\right)\right)
\end{aligned}
$$

For ${ }^{5} L_{J}(J=L-2)$, the one-loop operator is given by

$$
\begin{aligned}
& \int \frac{d \Omega}{4 \pi} S p {\left[V_{\mu_{1} \ldots \mu_{n}}^{(5) \alpha}\left(m_{1}+\hat{k}_{1}\right)\left(g_{\alpha \beta}^{\perp k_{1}}-\frac{\gamma_{\alpha}^{\perp k_{1}} \gamma_{\beta}^{\perp k_{1}}}{3}\right)\right.} \\
&\left.\times V_{\nu_{1} \ldots \nu_{n}}^{(5) \beta}\left(m_{2}-\hat{k}_{2}\right)\right]= \\
& \alpha_{n}|\boldsymbol{k}|^{2 n+4}(-1)^{n} O_{\nu_{1} \ldots \nu_{n}}^{\mu_{1} \ldots \mu_{n}} \\
& \times\left(\frac{(2 n+3) a_{1}}{(n+1)(n+2)}+\frac{9}{4}\left(-\frac{a_{2}|\boldsymbol{k}|^{2}}{n+1}+\frac{a_{3}|\boldsymbol{k}|^{4}}{2 n+1}\right)\right),
\end{aligned}
$$

while for ${ }^{5} L_{J}(J=L)$, the operator is written as

$$
\begin{aligned}
& \int \frac{d \Omega}{4 \pi} S p\left[V_{\mu_{1} \ldots \mu_{n}}^{(6) \alpha}\left(m_{1}+\hat{k}_{1}\right)\left(g_{\alpha \beta}^{\perp k_{1}}-\frac{\gamma_{\alpha}^{\perp k_{1}} \gamma_{\beta}^{\perp k_{1}}}{3}\right)\right. \\
& \left.\quad \times V_{\nu_{1} \ldots \nu_{n}}^{(6) \beta}\left(m_{2}-\hat{k}_{2}\right)\right]= \\
& \frac{\alpha_{n-1}}{2 n(2 n+1)}|\boldsymbol{k}|^{2 n}(-1)^{n} O_{\nu_{1} \ldots \nu_{n}}^{\mu_{1} \ldots \mu_{n}} \\
& \times\left[\frac{(2 n+3)(n+1) a_{1}}{3 n}-\frac{9}{8}|\boldsymbol{k}|^{2} a_{2}\left(\frac{2 n+5}{9}+\frac{2 n+1}{n(2 n-1)}\right)\right. \\
& \left.\quad+\frac{a_{3}|\boldsymbol{k}|^{4}(n+1)^{2}}{2(2 n-1)}\right] .
\end{aligned}
$$

The one-loop transition diagram between ${ }^{5} L_{J}(J=L+2)$ and ${ }^{5} L_{J}(J=L-2)$ states can be expressed as

$$
\begin{aligned}
& \int \frac{d \Omega}{4 \pi} S p\left[V_{\mu_{1} \ldots \mu_{n}}^{(4) \alpha}\left(m_{1}+\hat{k}_{1}\right)\left(g_{\alpha \beta}^{\perp k_{1}}-\frac{\gamma_{\alpha}^{\perp k_{1}} \gamma_{\beta}^{\perp k_{1}}}{3}\right)\right. \\
& \left.\times V_{\nu_{1} \ldots \nu_{n}}^{(5) \beta}\left(m_{2}-\hat{k}_{2}\right)\right]=\frac{9}{4} \frac{\alpha_{n-2}}{2 n+1} a_{3}|\boldsymbol{k}|^{2 n+4}(-1)^{n} O_{\nu_{1} \ldots \nu_{n}}^{\mu_{1} \ldots \mu_{n}}
\end{aligned}
$$

and the one-loop transition diagram between ${ }^{5} L_{J}(J=$ $L+2)$ and ${ }^{5} L_{J}(J=L)$ states as

$$
\begin{aligned}
& \int \frac{d \Omega}{4 \pi} S p {\left[V_{\mu_{1} \ldots \mu_{n}}^{(4) \alpha}\left(m_{1}+\hat{k}_{1}\right)\left(g_{\alpha \beta}^{\perp k_{1}}-\frac{\gamma_{\alpha}^{\perp k_{1}} \gamma_{\beta}^{\perp k_{1}}}{3}\right)\right.} \\
& \times\left.V_{\nu_{1} \ldots \nu_{n}}^{(6) \beta}\left(m_{2}-\hat{k}_{2}\right)\right]= \\
& \frac{3 \alpha_{n-2}(n+1)}{8(2 n+1)(2 n-1)}|\boldsymbol{k}|^{2 n}(-1)^{n} O_{\nu_{1} \ldots \nu_{n}}^{\mu_{1} \ldots \mu_{n}} \\
& \times\left(\frac{2 n+3}{n} a_{2}-2|\boldsymbol{k}|^{2} a_{3}\right) .
\end{aligned}
$$

For the one-loop transition diagram between ${ }^{5} L_{J}(J=$ $L-2)$ and ${ }^{5} L_{J}(J=L)$ we get

$$
\begin{aligned}
& \int \frac{d \Omega}{4 \pi} S p {\left[V_{\mu_{1} \ldots \mu_{n}}^{(5) \alpha}\left(m_{1}+\hat{k}_{1}\right)\left(g_{\alpha \beta}^{\perp k_{1}}-\frac{\gamma_{\alpha}^{\perp k_{1}} \gamma_{\beta}^{\perp k_{1}}}{3}\right)\right.} \\
&\left.\times V_{\nu_{1} \ldots \nu_{n}}^{(6) \beta}\left(m_{2}-\hat{k}_{2}\right)\right]= \\
& \frac{3 \alpha_{n}}{8(2 n+1)}|\boldsymbol{k}|^{2 n+4}(-1)^{n} O_{\nu_{1} \ldots \nu_{n}}^{\mu_{1} \ldots \mu_{n}}\left(a_{2}-2|\boldsymbol{k}|^{2} a_{3} \frac{n+1}{2 n-1}\right),
\end{aligned}
$$

and, for ${ }^{5} L_{J}(J=L-1)$, we find to

$$
\begin{aligned}
& \int \frac{d \Omega}{4 \pi} S p\left[V_{\mu_{1} \ldots \mu_{n}}^{(7) \alpha}\left(m_{1}+\hat{k}_{1}\right) \operatorname{Big}\left(g_{\alpha \beta}^{\perp k_{1}}-\frac{\gamma_{\alpha}^{\perp k_{1}} \gamma_{\beta}^{\perp k_{1}}}{3}\right)\right. \\
& \left.\quad \times V_{\nu_{1} \ldots \nu_{n}}^{(7) \beta}\left(m_{2}-\hat{k}_{2}\right)\right]= \\
& \frac{s \alpha_{n-1}}{2(2 n+1)}|\boldsymbol{k}|^{2 n+2}(-1)^{n} O_{\nu_{1} \ldots \nu_{n}}^{\mu_{1} \ldots \mu_{n}} \\
& \times\left(\frac{a_{1}(n+1)\left(2 n^{2}+n-2\right)}{n^{2}(2 n-1)}-\frac{9}{8}|\boldsymbol{k}|^{2} a_{2} \frac{n+1}{2 n-1}\right) \cdot
\end{aligned}
$$

Finally, the operator one-loop diagram For ${ }^{5} L_{J}(J=L+1)$ is equal to

$$
\begin{aligned}
& \int \frac{d \Omega}{4 \pi} S p\left[V_{\mu_{1} \ldots \mu_{n}}^{(8) \alpha}\left(m_{1}+\hat{k}_{1}\right)\left(g_{\alpha \beta}^{\perp k_{1}}-\frac{\gamma_{\alpha}^{\perp k_{1}} \gamma_{\beta}^{\perp k_{1}}}{3}\right)\right. \\
& \left.\times V_{\nu_{1} \ldots \nu_{n}}^{(8) \beta}\left(m_{2}-\hat{k}_{2}\right)\right]= \\
& \frac{s \alpha_{n-2}(n+1)}{2(2 n-1)(2 n-3))}|\boldsymbol{k}|^{2 n-2}(-1)^{n} O_{\nu_{1} \ldots \nu_{n}}^{\mu_{1} \ldots \mu_{n}} \\
& \times\left(a_{1}-\frac{9}{8}|\boldsymbol{k}|^{2} a_{2} \frac{n-1}{2 n+1}\right),
\end{aligned}
$$

and the one-loop transition diagram between ${ }^{5} L_{J}(J=$ $L-1)$ and ${ }^{5} L_{J}(J=L+1)$ can be written as

$$
\begin{gathered}
\int \frac{d \Omega}{4 \pi} S p\left[V_{\mu_{1} \ldots \mu_{n}}^{(7) \alpha}\left(m_{1}+\hat{k}_{1}\right)\left(g_{\alpha \beta}^{\perp k_{1}}-\frac{\gamma_{\alpha}^{\perp k_{1}} \gamma_{\beta}^{\perp k_{1}}}{3}\right)\right. \\
\left.\times V_{\nu_{1} \ldots \nu_{n}}^{(8) \beta}\left(m_{2}-\hat{k}_{2}\right)\right]= \\
\frac{s \alpha_{n-2}}{4 n^{2}-1}|\boldsymbol{k}|^{2 n}(-1)^{n} O_{\nu_{1} \ldots \nu_{n}}^{\mu_{1} \ldots \mu_{n}}\left(\frac{n+1}{n} a_{1}+\frac{9}{16}|\boldsymbol{k}|^{2} a_{2}(n+1)\right) .
\end{gathered}
$$




\section{Appendix 5. Amplitude of the triangle diagram}

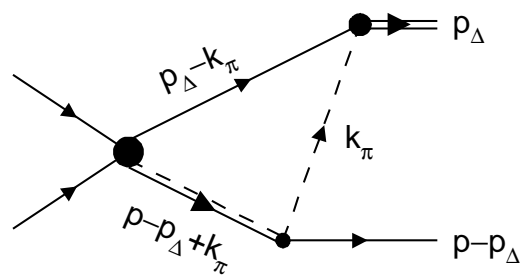

Fig. 7. Triangle diagram.

In the last two appendices, we give results on triangle diagrams in numerical form. First, we calculate the triangle-diagram integral which enters Eq. (126):

$$
\begin{aligned}
& A_{\text {triangle }}^{\text {spinless }}\left(W^{2}, s\right)=\int \frac{d^{4} k_{\pi}}{i(2 \pi)^{4}} \frac{1}{m_{\pi}^{2}-k_{\pi}^{2}-i 0} \\
& \times \frac{1}{m_{\Delta}^{2}-\left(p-p_{\Delta}+k_{\pi}\right)^{2}-i m_{\Delta} \Gamma_{\Delta}} \frac{1}{m_{N}^{2}-\left(p_{\Delta}-k_{\pi}\right)^{2}-i 0} .
\end{aligned}
$$

Notations of the momenta are illustrated by Fig. 7 . Here,

$$
p=p_{1}+p_{2}, \quad p^{2}=W^{2}, \quad p_{\Delta}^{2}=s .
$$

The physical region is located in the interval:

$$
\left(m_{N}+m_{\pi}\right)^{2} \leq s \leq\left(W-m_{N}\right)^{2} .
$$

The triangle-diagram amplitude $A_{\text {triangle }}^{\text {spinless }}\left(W^{2}, s\right)$ determined by (198) is shown in the physical region (200) in Fig. 8 (left column). In the right column, there are positions of the logarithmic singularities on the second sheet of the complex-s plane. Physical region of the reaction is also shown (thick solid line): it is located on the lower edge of the cut related to the threshold singularity (thin solid line). The positions of logarithmic singularities read:

$$
\begin{aligned}
& s_{( \pm)}^{(t r)}=m_{\pi}^{2}+m_{N}^{2}+\frac{\left(W^{2}-M_{\Delta}^{2}-m_{N}^{2}\right)\left(M_{\Delta}^{2}+m_{\pi}^{2}-m_{N}^{2}\right)}{2 M_{\Delta}^{2}} \\
& \pm\left[\left(m_{\pi}^{2}-\left(M_{\Delta}-m_{N}\right)^{2}\right)\left(m_{\pi}^{2}-\left(M_{\Delta}+m_{N}\right)^{2}\right)\right. \\
& \left.\times\left(W^{2}-\left(M_{\Delta}-m_{N}\right)^{2}\right)\left(W^{2}-\left(M_{\Delta}+m_{N}\right)^{2}\right)\right]^{1 / 2} \cdot(201)
\end{aligned}
$$

Here $M_{\Delta}^{2}=m_{\Delta}^{2}-i m_{\Delta} \Gamma_{\Delta}$.

In the left column of Fig. 8, the real and imaginary parts of the amplitude (198) at different total energies $W$ are shown by solid and dashed curves, respectively. In the right column, one sees the singularity positions, $s_{(-)}^{(t r)}$ (black circles) and $s_{(+)}^{(t r)}$ (black squares). When $s_{(+)}^{(t r)}$ dives into the third sheet, its position is shown as an open square.

\section{Appendix 6. Amplitude of the box diagram}

Here, we calculate the box-diagram integral which enters Eq. (136), the notations of momenta are given in Fig. 9 .
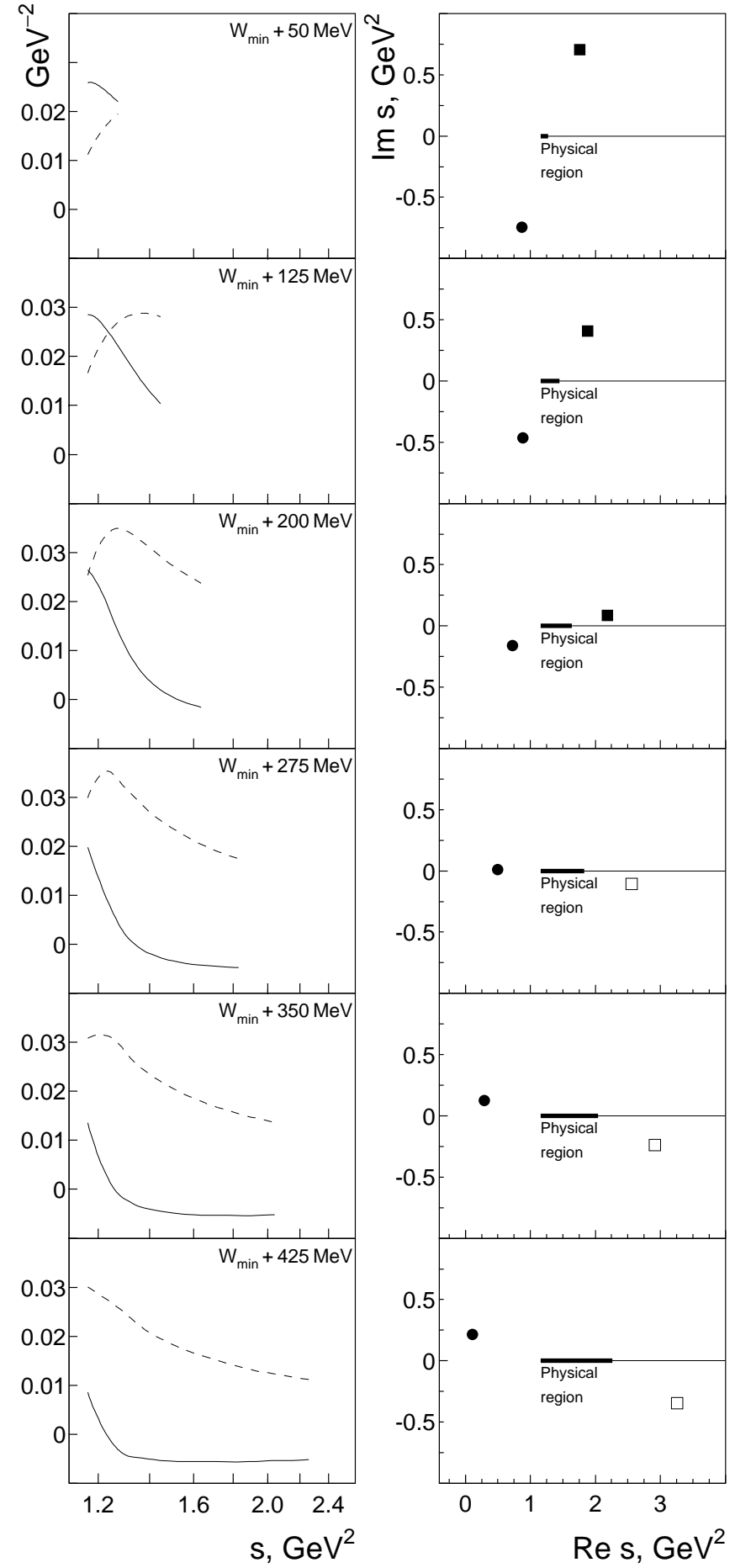

Fig. 8. Triangle diagram amplitude. In the left columns, real and imaginary parts of the amplitude are shown by solid and dashed curves, correspondingly. Initial energy, $W$, is shown on the top of each panel. In the right columns, singularity positions, $s_{( \pm)}^{(t r)}$, Eq.(201), are shown on the 2nd sheet of the complex-s plane. When $s_{(+)}^{(t r)}$ dives to the 3rd sheet, its position is shown by open square. 


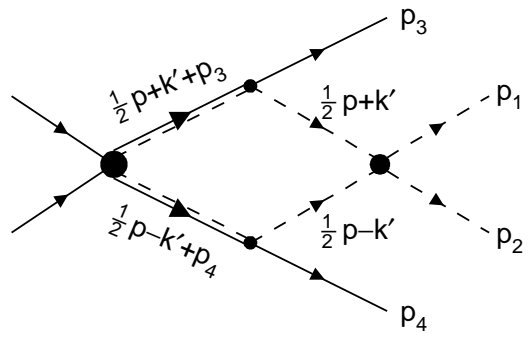

Fig. 9. Box diagram.

$$
\begin{aligned}
& A_{\text {box }}^{\text {spinless }}\left(W^{2}, s_{3}, s_{4}, s_{12}\right)= \\
& \int \frac{d^{4} k^{\prime}}{i(2 \pi)^{4}} \frac{1}{\left(m_{\Delta}^{2}-\left(\frac{1}{2} p+k^{\prime}+p_{3}\right)^{2}-i m_{\Delta} \Gamma_{\Delta}\right)} \\
& \times \frac{1}{\left(m_{\Delta}^{2}-\left(\frac{1}{2} p-k^{\prime}+p_{4}\right)^{2}-i m_{\Delta} \Gamma_{\Delta}\right)} \\
& \times \frac{1}{\left(m_{\pi}^{2}-\left(\frac{1}{2} p+k^{\prime}\right)^{2}-i 0\right)\left(m_{\pi}^{2}-\left(\frac{1}{2} p-k^{\prime}\right)^{2}-i 0\right)} .
\end{aligned}
$$

Remind that $s_{3}=\left(p-p_{3}\right)^{2}, s_{4}=\left(p-p_{4}\right)^{2}, s_{12}=\left(p_{1}+p_{2}\right)^{2}$, $W^{2}=p^{2}$.

In Fig. 10, we show the results of our calculation of $A_{\text {box }}^{\text {spinless }}\left(W^{2}, s_{3}, s_{4}, s_{12}\right)$ as a function of pion-pion energy squared $s_{12}$ at different total energies $W$, under the following constraint on $s_{3}$ and $s_{4}$ :

$$
s_{3}=s_{4}=\sqrt{s_{12}} W+m_{N}^{2} .
$$

This constraint corresponds to the following kinematics in the c.m. system:

$$
\begin{aligned}
p= & (W ; 0 ; 0 ; 0), \\
p_{1}= & \left(\sqrt{m_{\pi}^{2}+p_{1 z}^{2}} ; 0 ; 0 ; p_{1 z}\right), \\
p_{2}= & \left(\sqrt{m_{\pi}^{2}+p_{1 z}^{2}} ; 0 ; 0 ;-p_{1 z}\right), \\
p_{3}= & \left(\sqrt{m_{N}^{2}+p_{3 z}^{2}} ; 0 ; 0 ; p_{3 z}\right), \\
p_{4}= & \left(\sqrt{m_{N}^{2}+p_{3 z}^{2}} ; 0 ; 0 ;-p_{3 z}\right), \\
& \sqrt{m_{\pi}^{2}+p_{1 z}^{2}}+\sqrt{m_{N}^{2}+p_{3 z}^{2}}=W / 2
\end{aligned}
$$

The positions of the box-diagram singularities are given by the formula:

$$
\begin{aligned}
& s_{12}^{\mathrm{box}}=2 m_{\pi}^{2}+\frac{1}{2 W^{2}}\left(s_{3}-m_{N}^{2}\right)\left(s_{4}-m_{N}^{2}\right) \\
+ & \frac{\left(2 W^{2} M_{\Delta}^{2}-W^{2}\left(s_{3}-m_{N}^{2}\right)\right)\left(2 W^{2} M_{\Delta}^{2}-W^{2}\left(s_{4}-m_{N}^{2}\right)\right)}{2 W^{2}\left(\left(W^{2}-2 M_{\Delta}^{2}\right)^{2}-4 M_{\Delta}^{4}\right)} \\
- & {\left[\left(\frac{\left(s_{3}-m_{N}^{2}\right)^{2}}{2 W^{2}}-2 m_{\pi}^{2}-\frac{\left(2 W^{2} M_{\Delta}^{2}-W^{2}\left(s_{3}-m_{N}^{2}\right)\right)^{2}}{2 W^{2}\left(\left(W^{2}-2 M_{\Delta}^{2}\right)^{2}-4 M_{\Delta}^{4}\right)}\right)\right.} \\
\times & \left.\left(\frac{\left(s_{4}-m_{N}^{2}\right)^{2}}{2 W^{2}}-2 m_{\pi}^{2}-\frac{\left(2 W^{2} M_{\Delta}^{2}-W^{2}\left(s_{4}-m_{N}^{2}\right)\right)^{2}}{2 W^{2}\left(\left(W^{2}-2 M_{\Delta}^{2}\right)^{2}-4 M_{\Delta}^{4}\right)}\right)\right]^{\frac{1}{2}} .
\end{aligned}
$$

At $s_{3}=s_{4}$, Eq. (205) reads

$$
s_{12}^{\mathrm{box}}=4 m_{\pi}^{2}+\frac{W^{2}\left(2 M_{\Delta}^{2}-s_{3}+m_{N}^{2}\right)^{2}}{\left(W^{2}-2 M_{\Delta}^{2}\right)^{2}-4 M_{\Delta}^{4}} .
$$

Recall that in (205) and (206) $M_{\Delta}^{2}$ is given by

$$
M_{\Delta}^{2}=m_{\Delta}^{2}-i m_{\Delta} \Gamma_{\Delta}
$$
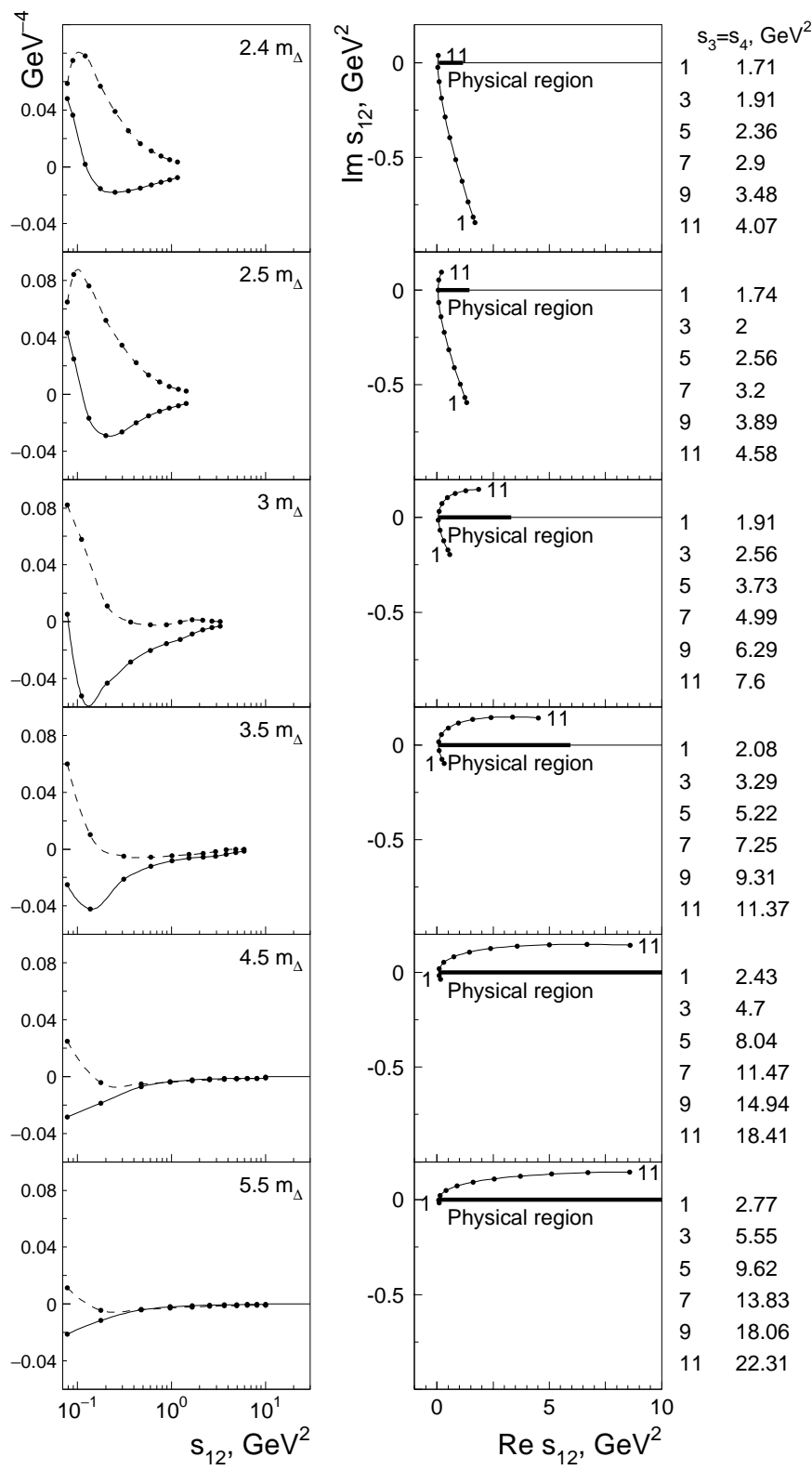

Fig. 10. Box diagram amplitude as a function of $s_{12}$ under the constraint (203) (corresponding magnitudes of $s_{3}$ and $s_{4}$ are shown in the right column. In the left columns, real and imaginary parts of the amplitude are shown by solid and dashed curves correspondingly. Initial energy, $W$, is shown on the top of each panel. On the right columns singularity positions, $s_{12}^{\text {box }}$, Eq.(205), are shown on the 2 nd sheet of the complex- $s_{12}$ plane.

\section{References}

1. D. J. Gross and F. Wilczek, Phys. Rev. Lett. 30 (1973) 1343. 
2. S. Weinberg, Nucl. Phys. B 363 (1991) 3.

3. S. Aoki et al., Phys. Rev. Lett. 84 (2000) 238.

4. C. Amsler and N. A. Tornqvist, Phys. Rept. 389 (2004) 61.

5. D. V. Bugg, Phys. Rept. 397 (2004) 257.

6. V. V. Anisovich, Int. J. Mod. Phys. A 21 (2006) 3615 arXiv:hep-ph/0510409.

7. E. Klempt and A. Zaitsev, "Glueballs, hybrids, multiquarks," submitted to Phys. Rept. (2007).

8. C. Amsler et al., Phys. Lett. B 322 (1994) 431.

9. V. V. Anisovich et al., Phys. Lett. B 323 (1994) 233.

10. C. Amsler et al., Phys. Lett. B 333 (1994) 277.

11. C. Amsler et al., Phys. Lett. B 340 (1994) 259.

12. C. Amsler et al., Phys. Lett. B 355 (1995) 425.

13. C. Amsler et al., Phys. Lett. B 342 (1995) 433.

14. C. Amsler et al., Phys. Lett. B 353 (1995) 571.

15. A. V. Anisovich et al., Phys. Lett. B 491 (2000) 47.

16. A. V. Anisovich et al., Phys. Lett. B 517 (2001) 261.

17. A. V. Anisovich et al., Phys. Lett. B 542 (2002) 8.

18. A. V. Anisovich et al., Phys. Lett. B 542 (2002) 19.

19. A. V. Anisovich, V. V. Anisovich and A. V. Sarantsev, Phys. Rev. D 62 (2000) 051502(R).

20. S. Godfrey and N. Isgur, Phys. Rev. D 32 (1985) 189.

21. U. Loring, B. C. Metsch and H. R. Petry, Eur. Phys. J. A 10 (2001) 395 arXiv:hep-ph/0103289.

22. U. Loring, B. C. Metsch and H. R. Petry, Eur. Phys. J. A 10 (2001) 447 arXiv:hep-ph/0103290.

23. A. Karch et al., Phys. Rev. D 74 (2006) 015005.

24. E. Klempt, Phys. Rev. C 66 (2002) 058201.

25. O. Bartholomy et al., Phys. Rev. Lett. 94 (2005) 012003.

26. V. Crede et al., Phys. Rev. Lett. 94 (2005) 012004.

27. B. Krusche et al., Phys. Rev. Lett. 74 (1995) 3736.

28. O. Bartalini et al., Eur. Phys. J. A 26 (2005) 399.

29. A. A. Belyaev et al., Nucl. Phys. B 213 (1983) 201.

30. R. Beck et al., Phys. Rev. Lett. 78 (1997) 606.

31. D. Rebreyend et al., Nucl. Phys. A 663 (2000) 436.

32. J. Ajaka et al., Phys. Rev. Lett. 81 (1998) 1797.

33. K. H. Althoff et al., Z. Phys. C 18 (1983) 199.

34. E. J. Durwen, Bonn-IR-80-7 (1980).

35. K. Buechler et al., Nucl. Phys. A 570 (1994) 580.

36. K. H. Glander et al., Eur. Phys. J. A 19 (2004) 251.

37. J. W. C. McNabb et al., Phys. Rev. C 69 (2004) 042201.

38. R. G. T. Zegers et al., Phys. Rev. Lett. 91 (2003) 092001.

39. R. Lawall et al., Eur. Phys. J. A 24 (2005) 275.

40. A. Braghieri et al., Phys. Lett. B 363 (1995) 46.

41. F. Harter et al., Phys. Lett. B 401 (1997) 229.

42. M. Wolf et al., Eur. Phys. J. A 9 (2000) 5.

43. J. Ahrens et al. [GDH and A2 Collaborations], Phys. Lett. B 624 (2005) 173.

44. Y. Assafiri et al., Phys. Rev. Lett. 90 (2003) 222001.

45. M. Ripani et al., Phys. Rev. Lett. 91 (2003) 022002.

46. S. Strauch et al., Phys. Rev. Lett. 95 (2005) 162003.

47. C. Wu et al., Eur. Phys. J. A 23 (2005) 317.

48. U. Thoma et al., "N* and $\Delta^{*}$ decays into $\mathrm{N} \pi^{0} \pi^{0}$ ", submittd to PRL (2007).

49. J. Barth et al., Eur. Phys. J. A 18 (2003) 117.

50. E. Hourany et al., Nucl. Phys. A 755 (2005) 447.

51. R. Bradford et al. [CLAS Collaboration], Phys. Rev. C 75, 035205 (2007) arXiv:nucl-ex/0611034.

52. A. Lleres et al., Eur. Phys. J. A 31, 79 (2007).

53. A. V. Anisovich et al., Eur. Phys. J. A 25 (2005) 427.

54. A. V. Sarantsev et al., Eur. Phys. J. A 25 (2005) 441.

55. E. Klempt et al., Eur. Phys. J. A 29 (2006) 307.
56. D. Diakonov, V. Petrov and M. V. Polyakov, Z. Phys. A 359 (1997) 305.

57. R. L. Jaffe and F. Wilczek, Phys. Rev. Lett. 91 (2003) 232003.

58. T. Nakano et al., Phys. Rev. Lett. 91 (2003) 012002.

59. S. Stepanyan et al., Phys. Rev. Lett. 91 (2003) 252001.

60. V. V. Barmin et al., Phys. Atom. Nucl. 66 (2003) 1715 [Yad. Fiz. 66 (2003) 1763].

61. J. Barth et al., Phys. Lett. B 572 (2003) 127.

62. M. Abdel-Bary et al., Phys. Lett. B 595 (2004) 127.

63. M. Abdel-Bary et al., "Improved study of a possible $\Theta^{+}$ production in the $p p \rightarrow p K^{0} \Sigma^{+}$reaction with the COSYTOF spectrometer," arXiv:hep-ex/0612048

64. J. T. Balewski et al., Phys. Lett. B 388 (1996) 859.

65. A. Bondar et al., Phys. Lett. B 356 (1995) 8.

66. H. Calen et al., Phys. Lett. B 366 (1996) 39.

67. H. Calen et al., Phys. Rev. Lett. 79 (1997) 2642.

68. J. Zlomanczuk et al., Phys. Lett. B 436 (1998) 251.

69. A. Betsch et al., Phys. Lett. B 446 (1999) 179.

70. H. Calen et al., Phys. Lett. B 458 (1999) 190.

71. S. Sewerin et al., Phys. Rev. Lett. 83 (1999) 682.

72. H. Calen et al., Phys. Rev. Lett. 80 (1998) 2069.

73. P. Moskal et al., Phys. Rev. Lett. 80 (1998) 3202.

74. J. T. Balewski et al., Phys. Lett. B 420 (1998) 211.

75. H. Calen et al., Phys. Rev. C 58 (1998) 2667.

76. P. Moskal et al., Phys. Lett. B 474 (2000) 416.

77. F. Balestra et al., Phys. Lett. B 491 (2000) 29

78. J. Greiff et al., Phys. Rev. C 62 (2000) 064002.

79. P. Moskal et al., Phys. Lett. B 482 (2000) 356.

80. S. Abd El-Samad et al., Phys. Lett. B 522 (2001) 16.

81. C. Quentmeier et al., Phys. Lett. B 515 (2001) 276.

82. P. Moskal et al., Phys. Lett. B 517 (2001) 295.

83. R. Bilger et al., Nucl. Phys. A 693, 633 (2001).

84. W. Brodowski et al., Phys. Lett. B 550 (2002) 147.

85. P. Winter et al., Phys. Lett. B 544 (2002) 251 [Erratumibid. B 553 (2003) 339].

86. J. Greiff et al., Phys. Rev. C 65 (2002) 034009.

87. P. Moskal, M. Wolke, A. Khoukaz and W. Oelert, Prog. Part. Nucl. Phys. 49 (2002) 1.

88. M. Abdel-Bary et al., Eur. Phys. J. A 16 (2003) 127.

89. W. Brodowski et al., Phys. Rev. Lett. 88 (2002) 192301.

90. L. A. Kondratyuk et al., Phys. Atom. Nucl. 66 (2003) 152 [Yad. Fiz. 66 (2003) 155].

91. P. Moskal et al., Phys. Rev. C 69 (2004) 025203.

92. M. Abdel-Bary et al., Phys. Rev. C 68 (2003) 021603.

93. V. Kleber et al., Phys. Rev. Lett. 91 (2003) 172304.

94. J. Patzold et al., Phys. Rev. C 67 (2003) 052202.

95. S. Yaschenko et al., Phys. Rev. Lett. 94 (2005) 072304.

96. M. Abdel-Bary et al., Phys. Lett. B 610 (2005) 31.

97. V. Y. Grishina et al., Eur. Phys. J. A 21 (2004) 507.

98. M. Abdel-Bary et al., arXiv:hep-ex/0512033.

99. V. Kleber, Int. J. Mod. Phys. A 20 (2005) 273.

100. A. Wronska and V. Hejny, Int. J. Mod. Phys. A 20 (2005) 640 [Acta Phys. Slov. 56 (2005) 279].

101. Yu. Valdau, Int. J. Mod. Phys. A 20 (2005) 677.

102. S. Dymov et al., Phys. Lett. B 635 (2006) 270.

103. I. Zychor et al., Phys. Rev. Lett. 96 (2006) 012002.

104. P. Moskal et al., J. Phys. G 32 (2006) 629.

105. M. Abdel-Bary et al., Phys. Lett. B 619 (2005) 281.

106. A. Dzyuba et al., Eur. Phys. J. A 29 (2006) 245.

107. M. Abdel-Bary et al., Eur. Phys. J. A 29 (2006) 353.

108. T. Rozek et al., Phys. Lett. B 643 (2006) 251.

109. S. Barsov et al., arXiv:nucl-ex/0609010. 
110. P. Winter et al., Phys. Lett. B 635 (2006) 23.

111. Y. Maeda et al., Phys. Rev. Lett. 97 (2006) 142301.

112. S. A. El-Samad et al., Eur. Phys. J. A 30 (2006) 443.

113. Yu. N. Uzikov, J. Haidenbauer and C. Wilkin, Phys. Rev. C 75 (2007) 014008.

114. M. Hartmann et al., Phys. Rev. Lett. 96 (2006) 242301 [Erratum-ibid. 97 (2006) 029901].

115. M. Abdel-Bary et al., "Comparison of isoscalar vector meson production cross sections in proton proton collisions," arXiv:nucl-ex/0702059

116. H. H. Adam et al. [WASA-at-COSY Collaboration], "Proposal for the Wide Angle Shower Apparatus (WASA) at COSY-Jülich - 'WASA at COSY'," arXiv:nucl-ex/0411038

117. C. Hanhart et al., Phys. Lett. B 424 (1998) 8.

118. C. Hanhart and K. Nakayama, Phys. Lett. B 454 (1999) 176.

119. V. Bernard, N. Kaiser and U. G. Meissner, Eur. Phys. J. A 4 (1999) 259.

120. L. Alvarez-Ruso, Phys. Lett. B 452 (1999) 207.

121. C. Hanhart et al., Phys. Lett. B 444 (1998) 25.

122. J. A. Niskanen, Phys. Lett. B 456 (1999) 107.

123. V. Dmitrasinovic et al., Phys. Lett. B 465 (1999) 43.

124. N. Kaiser, Eur. Phys. J. A 5 (1999) 105.

125. N. Kaiser, Phys. Rev. C 60 (1999) 057001.

126. K. Nakayama et al., Phys. Rev. C 61 (2000) 024001.

127. H. Machner and J. Haidenbauer, J. Phys. G 25 (1999) R231.

128. R. Shyam, Phys. Rev. C 60 (1999) 055213.

129. R. Shyam, G. Penner and U. Mosel, Phys. Rev. C 63 (2001) 022202.

130. A. Sibirtsev et al., "On the $\Lambda$ to $\Sigma^{0}$ ratio from proton proton collisions," arXiv:nucl-th/0004022.

131. C. Hanhart and N. Kaiser, Phys. Rev. C 66 (2002) 054005 .

132. K. Nakayama, J. Speth and T. S. H. Lee, Phys. Rev. C 65 (2002) 045210.

133. V. Baru et al., Phys. Rev. C 67 (2003) 024002.

134. K. Nakayama et al., Phys. Rev. C 68 (2003) 045201.

135. C. Hanhart, Phys. Rept. 397 (2004) 155.

136. A. Deloff, Phys. Rev. C 69 (2004) 035206.

137. C. Hanhart et al., Phys. Lett. B 590 (2004) 39.

138. K. Nakayama and H. Haberzettl, Phys. Rev. C 69 (2004) 065212.

139. C. Hanhart et al., Phys. Lett. B 606 (2005) 67.

140. V. Baru et al., Eur. Phys. J. A 23 (2005) 523.

141. V. Lensky et al., Eur. Phys. J. A 27 (2006) 37.

142. R. Shyam, Phys. Rev. C 73 (2006) 035211.

143. S. Schneider, S. Krewald and U. G. Meissner, Eur. Phys. J. A 28 (2006) 107.

144. A. Sibirtsev et al., Eur. Phys. J. A 27 (2006) 269.

145. A.V. Anisovich et al., J. Phys. G 28 (2002) 15.

146. V.V. Anisovich, A.V. Sarantsev and D.V. Bugg, Nucl. Phys. A537, 1385 (1991).

147. A.V. Anisovich et al., Eur. Phys. J. A 24 (2005) 111.

148. A.V. Anisovich and A.V. Sarantsev, Eur. Phys. J. A 30 427 (2006) 427.

149. M. Fierz, Zeit. Phys. 104 (1937) 553.

150. K.L. Au, D. Morgan and M.R. Pennington, Phys. Rev.D 35, 1633 (1987).

151. V.V. Anisovich, Yu.D. Prokoshkin and A.V. Sarantsev, Phys. Lett., B 389, 388 (1996).
152. V.V. Anisovich and A.V. Sarantsev, Phys. Lett., B 413, 137 (1997).

153. I.J.R. Aitchison, Phys. Rev. 133, 1257 (1964).

154. V.V. Anisovich and L.G. Dakhno, Phys. Lett. 10, 221 (1964).

155. V.V. Anisovich, Yad. Fiz. 6, 146 (1967).

156. P. Collas and R.E. Norton, Phys. Rev. 160, 1346 (1967).

157. V.V. Anisovich, M.N. Kobrinsky, J. Nyiri, Yu.M. Shabelski, "Quark model and high energy collisions", second edition, World Scientific, 2004. 


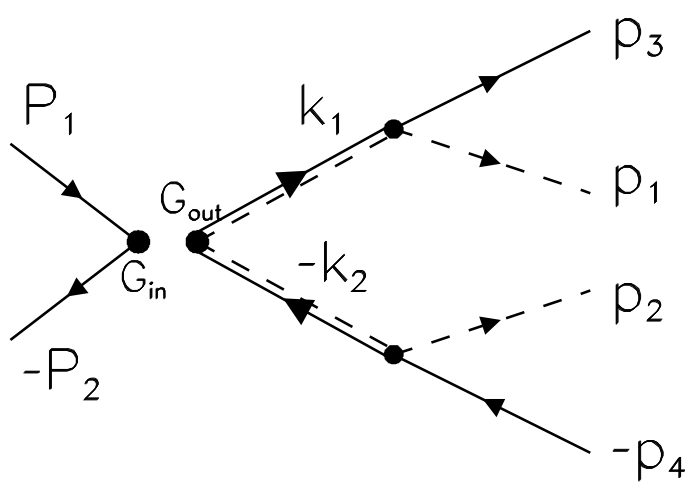

